\title{
EXPLORATION OF MAGNETISM IN 3D AND 2D SYSTEMS
}

A Dissertation presented to

the Doctoral Committee

at the University of Missouri

In Partial Fulfillment

of the Requirements for the Degree

Doctor of Philosophy

by

ASHUTOSH DAHAL

Dr. Deepak K. Singh, Dissertation Supervisor

JULY 2019 
The undersigned, appointed by the Dean of the Graduate School, have examined the dissertation entitled:

\section{EXPLORATION OF MAGNETISM IN 3D AND 2D SYSTEMS}

presented by Ashutosh Dahal, a candidate for the degree of Doctor of Philosophy and hereby certify that, in their opinion, it is worthy of acceptance.

\begin{tabular}{l}
\hline Dr. Deepak K. Singh \\
\hline Dr. Paul F. Miceli \\
\hline Dr. David J. Singh \\
\hline
\end{tabular}

Dr. J. David Robertson 


\section{ACKNOWLEDGMENTS}

I am grateful to my adviser, Dr. Deepak K. Singh for I have been fortunate to work with him. Only through his support and guidance was I able to finish my Ph.D. study.

I would like to thank Drs. Paul F. Miceli, David J. Singh, and J. David Robertson for serving on my Doctoral Committee. My thanks also goes to Dr. Tom Heitmann from University of Missouri Research Reactor (MURR)and to Dr. Leland Harrigar

and Dr. Jose Rodriguez Riviera from NIST Center for Neutron Research for helping me with experiments.

I am very fortunate to have gotten the opportunity to work with Dr. Jagath Gunasekera, Dr. Brock Summers, Dr. Yiyao Chen and George Yumnam in research experiments together, solve problems and share lab work.

I would like to thank all my colleagues and friends, including Aayush Regmi, Milica Utjsanovic, Lisa Shepard, Julie Alsberg, Marat Musin, Vici Gruss, and Anna Kulynych for the camaraderie and support outside the lab.

Finally, I would like to express my gratitude to my father Ramhari Dahal, my mother Sharmila Koirala, and my sisters Astha and Asmita Dahal, without whom this PhD would be impossible. They have shaped my journey and I am here because of them. 


\section{TABLE OF CONTENTS}

ACKNOWLEDGMENTS ................ ii

LIST OF TABLES $\ldots \ldots \ldots \ldots \ldots \ldots$ vi

LIST OF FIGURES $\ldots \ldots \ldots \ldots \ldots \ldots$ vii

ABSTRACT ...................

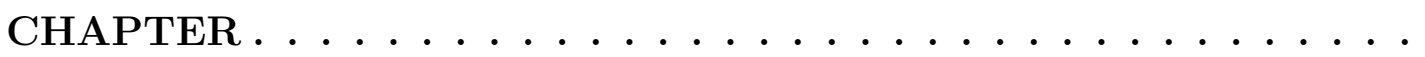

1 Introduction ...................... 1

1.1 Magnetic moment correlation and magnetic p- hase transition . . . . 1

1.2 Static and Dynamic magnetic moment correlation . . . . . . . . . 3

1.3 Monosilicides - Interesting case of Nickel Mo-nosilicide . . . . . . 6

1.4 Presence or absence of magnetic correlation in ruthenates . . . . . . 8

1.5 Static magnetic correlation in $\mathrm{EuMn}_{2} \mathrm{As}_{2} \ldots \ldots \ldots \ldots$

1.6 Magnetic phase transition in two-dimensional permalloy honeycomb lattice . . . . . . . . . . . . . . . . . 13

2 Study of Magnetism in NiSi . . . . . . . . . . . 16

2.1 Sample Fabrication $\ldots \ldots \ldots \ldots \ldots \ldots$

2.2 Structural Characterization _. . . . . . . . . . . . 18

2.3 Heat Capacity Measurements . . . . . . . . . . . . . . . 19

2.4 Electrical Measurements . . . . . . . . . . . . . . . . 23

2.5 Magnetization Measurements . . . . . . . . . . . 26

2.5.1 Test for Normal Skin Effect . . . . . . . . . . . . . . . 32

2.5.2 Neutron Scattering Measurement _. . . . . . . . . . 37

2.6 Results and Discussion . . . . . . . . . . . . . . 47 
3 Quantum Magnetic Properties in Co doped $\mathrm{CaRuO}_{3} \ldots \ldots$

3.1 Sample Preparation . . . . . . . . . . . . . . . . . . . . . 49

3.2 Sample Characterization . . . . . . . . . . . . . . 50

3.3 Electrical Measurements . . . . . . . . . . . . . . . . 52

3.4 Magnetization Measurements . . . . . . . . . . . . 53

3.5 Neutron Scattering Measurements . . . . . . . . . . . . . 55

3.5.1 Elastic Neutron Scattering Measurements . . . . . . . . . . . 55

3.5.2 Inelastic Neutron Scattering Measurements . . . . . . . . . . 57

3.6 Heat Capacity Measurements . . . . . . . . . . . . . . . . . 64

3.7 Results and Discussion . . . . . . . . . . . . . 66

4 Spin Correlation in EuMn $\mathrm{Eus}_{2} \ldots \ldots \ldots$

4.1 Structure of $\mathrm{EuMn}_{2} \mathrm{As}_{2} \ldots \ldots \ldots \ldots \ldots$

4.2 Neutron Scattering Measurements . . . . . . . . . . . . . 69

4.2.1 Elastic Neutron Scattering Measurements . . . . . . . . . . . 69

4.2.2 Absorption Correction for $\mathrm{Eu}$. . . . . . . . . . . . . 71

4.2.3 Order Parameter Measurements . . . . . . . . . . . . . 71

4.2.4 Numerical Modeling of Elastic Data . . . . . . . . . . . . . . 74

4.3 Results and Discussion . . . . . . . . . . . . . . . 78

5 Magnetism in Artificial Honeycomb Lattice of Py . . . . . . . 79

5.1 Sample Characterization . . . . . . . . . . . . . . . 81

5.2 Electrical Measurements . . . . . . . . . . . . . . . . 82

5.3 Magnetization Measurements . . . . . . . . . . 86

5.4 Results and Discussion . . . . . . . . . . . . . . . . . . 99

6 Summary and concluding remarks . . . . . . . . . . 101

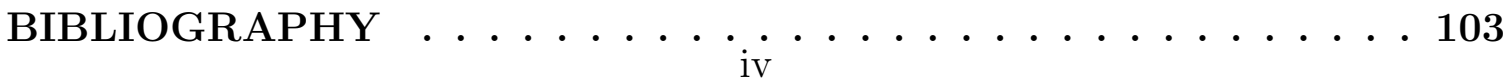




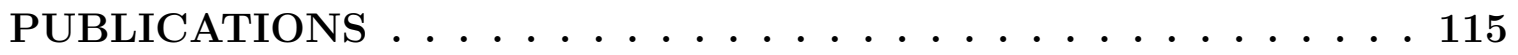

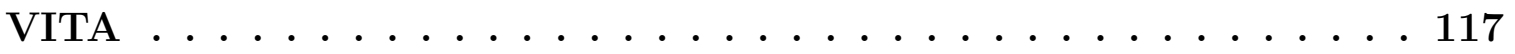




\section{LIST OF TABLES}

Table

4.1 Experimentally estimated and calculated intensities at half-integer magnetic wave vectors at $\mathrm{T}=6 \mathrm{~K}$. As shown in Fig. 4.5(a), the peak intensity at $[003 / 2]$ position is spurious in nature $\ldots \ldots \ldots 76$

4.2 Experimentally estimated and calculated intensities at integer magnetic wave vectors at $\mathrm{T}=6 \mathrm{~K} \ldots \ldots \ldots \ldots \ldots$ 


\section{LIST OF FIGURES}

Figure $\quad$ Page

1.1 Different Kinds of Magnetization . . . . . . . . . . . . 5

1.2 Hall measurement of $\mathrm{NiSi}$. . . . . . . . . . . . . . . . . . . . 7

1.3 NMR measurement of $\mathrm{CaRuO}_{3}$ at $4.2 \mathrm{~K} \ldots \ldots . \ldots . \ldots$

1.4 Inelastic neutron scattering measurements of $\mathrm{CaRuO}_{3}$ at different tem-

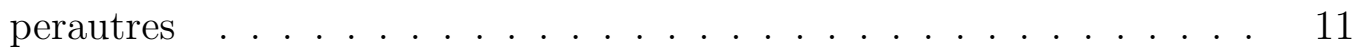

1.5 Heat Capacity measurement of $\mathrm{EuMn}_{2} \mathrm{As}_{2} \ldots \ldots$. . . . . . . . . 13

1.6 Phase diagram of Artificial Honeycomb lattice . . . . . . . . . . . . 15

2.1 NiSi Sample . . . . . . . . . . . . . . . . . . . . 17

2.2 X-Ray Diffraction Pattern of NiSi . . . . . . . . . . . . . . . 19

2.3 Schematics of Heat Capacity measurement of NiSi . . . . . . . . . . . 20

2.4 Heat Capacity measurement of $\mathrm{NiSi}$. . . . . . . . . . . . . . . . 21

2.5 Heat Capacity measurement fitting of NiSi . . . . . . . . . . . . . . . 22

2.6 Four Probe measurement technique . . . . . . . . . . . . . . . 23

2.7 Resistivity measurement of $\mathrm{NiSi} \ldots \ldots . . \ldots 24$

2.8 Resistivity measurement of NiSi with applied field . . . . . . . . . 25

2.9 AC susceptibility measurement of $\mathrm{NiSi} \ldots \ldots$. . . . . . . . . . 28

2.10 AC susceptibility measurement of NiSi at lower field . . . . . . . . . . 29

2.11 DC susceptibility measurement of $\mathrm{NiSi} \ldots$. . . . . . . . . . . . 31

2.12 Calculated resistivity of NiSi to test Skin Effect . . . . . . . . . . 33 vii 
$2.13 \chi^{\prime}$ and $\chi^{\prime \prime}$ deduced from the electrical resistance of NiSi . . . . . . 35

$2.14 \mathrm{AC}$ susceptibility measurements of NiSi mixed with $B_{4} C_{3}$ powder . . 36

2.15 Schematic of Neutron Scattering measurement . . . . . . . . 38

2.16 Elastic Neutron Scattering measurements for NiSi . . . . . . . . 40

2.17 Inelastic Neutron Scattering measurements in NiSi at 1.5 K . . . . . 42

2.18 Inelastic Neutron Scattering measurements in NiSi at multiple temper-

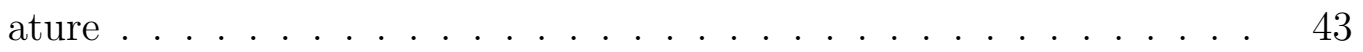

$2.19 \chi "(Q, E)$ derived from Inelastic Neutron Scattering measurements in $\mathrm{NiSi} \ldots \ldots \ldots \ldots \ldots \ldots \ldots \ldots \ldots \ldots$

2.20 Inelastic Neutron Scattering measurements in NiSi at different Q value 46

3.1 X-Ray Diffraction Pattern of $\mathrm{Ca}\left(\mathrm{Co}_{x} \mathrm{Ru}_{1-x}\right) \mathrm{O}_{3} \ldots \ldots \ldots \ldots$

3.2 Electrical resistivity measurement of $\mathrm{Ca}\left(\mathrm{Co}_{x} \mathrm{Ru}_{1-x}\right) \mathrm{O}_{3} \quad \ldots \ldots . . .52$

3.3 Magnetization measurements of $\mathrm{Ca}\left(\mathrm{Co}_{x} \mathrm{Ru}_{1-x}\right) \mathrm{O}_{3} \ldots \ldots \ldots \ldots$

3.4 Elastic Neutron Scattering measurements of $\mathrm{Ca}\left(\mathrm{Co}_{x} \mathrm{Ru}_{1 x}\right) \mathrm{O}_{3} \ldots \ldots$

3.5 Inelastic Neutron Scattering measurements of $\mathrm{Ca}\left(\mathrm{Co}_{x} \mathrm{Ru}_{1-x}\right) \mathrm{O}_{3}$ at SPINS 58

3.6 Inelastic measurements of $\mathrm{CaRuO}_{3} \quad \ldots \ldots \ldots \ldots$

3.7 Background data for Inelastic Neutron scattering measurements of $\mathrm{Ca}\left(\mathrm{Co}_{x} \mathrm{Ru}_{1-x}\right) \mathrm{O}_{3}$ at MACS $\ldots \ldots \ldots \ldots \ldots$

3.8 Inelastic Neutron Scattering measurements of $\mathrm{Ca}\left(\mathrm{Co}_{x} \mathrm{Ru}_{1 x}\right) \mathrm{O}_{3}$ at MACS 61

3.9 Heat Capacity measurements of $\mathrm{Ca}\left(\mathrm{Co}_{x} \mathrm{Ru}_{1 x}\right) \mathrm{O}_{3} \ldots \ldots \ldots$

4.1 Crystal Structure of $\mathrm{EuMn}_{2} \mathrm{As}_{2} \ldots \ldots \ldots \ldots \ldots$

4.2 Schematic description of elastic measurement . . . . . . . . 70

4.3 Order Parameter measurements of $\mathrm{EuMn}_{2} \mathrm{As}_{2} \quad \ldots \ldots \ldots \ldots$

4.4 Elastic measurements of $\mathrm{EuMn}_{2} \mathrm{As}_{2} \ldots \ldots \ldots \ldots \ldots$

4.5 Model of $\mathrm{EuMn}_{2} \mathrm{As}_{2} \ldots \ldots \ldots \ldots \ldots \ldots \ldots$

5.1 Moment configuration in Artificial Honeycomb lattice . . . . . . . . 80 viii 
5.2 AFM image of Honeycomb lattice . . . . . . . . . . . . . . 81

5.3 Electrical resistivity measurements of Artificial Honeycomb lattice . . 84

5.4 Conductivity data for Artificial Honeycomb lattice . . . . . . . . 85

5.5 Magnetization as a function of field in Artificial Honeycomb lattice . 88

$5.6 \mathrm{MvsH}$ measurements in Artificial Honeycomb lattice . . . . . . . . . 90

5.7 Nonlinear susceptibility of Artificial Honeycomb lattice . . . . . . . 91

$5.8 \chi_{3}$ as a function of temperature $\ldots \ldots \ldots \ldots \ldots \ldots$

5.9 Estimation of static critical exponents $\gamma$ and $\delta \ldots \ldots \ldots . \ldots 94$

5.10 Scaling analysis of nonlinear susceptibilities in artificial honeycomb

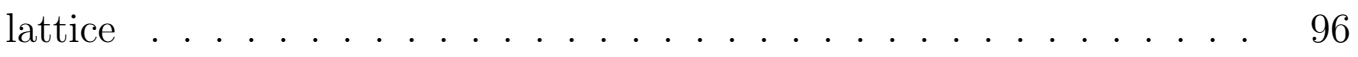

5.11 Scaling analysis of nonlinear susceptibilities using the estimated exponents of $|\gamma|=1.9, \delta=2.4$ and $|\beta|=1.4$ in high field regime

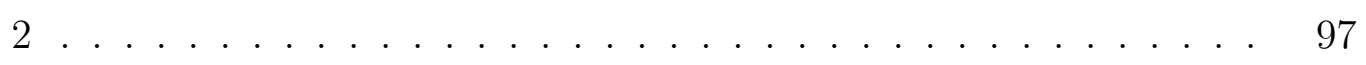

5.12 Scaling analysis of nonlinear susceptibilities, $\chi_{n 1}$, in low field regime 1 using the estimated exponents of $|\gamma|=1.4, \delta=2.5$ and $|\beta|=0.95 \quad . \quad 98$ 


\section{ABSTRACT}

Magnetism has intruded in every aspect of our life, from electric motors to hard disk data storage to space technologies. Developing strong understanding of underlying magnetic properties is of utmost importance to reach new frontiers of technological advancement. During my Ph.D. research, I have explored complementary research venues in three dimensional as well as two-dimensional materials to understand basic magnetic properties that were either not known or explored for the first time. In this quest, I have studied three different physical systems with overlapping structural and/or magnetic and electrical properties: nickel monosilicides $(\mathrm{NiSi})$, cobalt-doped calcium ruthenate $\left(\mathrm{Ca}\left(\mathrm{Co}_{x} \mathrm{Ru}_{1-x}\right) \mathrm{O}_{3}\right)$ and europium manganese arsenide $\left(\mathrm{EuMn}_{2} \mathrm{As}_{2}\right)$. One of the key aspects of my research is to understand how magnetic moments correlate with each other. Understanding this fundamental question can help us in elucidating the mechanism behind novel magnetic properties manifested by the above mentioned materials. While NiSi is found to manifest a new phenomenon of magnetism driven intermediate metallic-superconducting phase, $\mathrm{Ca}\left(\mathrm{Co}_{x} \mathrm{Ru}_{1-x}\right) \mathrm{O}_{3}$ tends to exhibit the metal-insulator transition with the critical phase boundary coinciding with the onset of strong continuum type magnetic fluctuations. Despite the presence of strong dynamic magnetic moment correlation, no trace of any type of static magnetic order is detected in any of these materials. On the other hand, strong static order with two consecutive antiferromagnetic phase transitions are detected in the intertwined honeycomb structured $\mathrm{EuMn}_{2} \mathrm{As}_{2}$.

During the process of studying bulk materials using macroscopic measurement techniques, I have acquired detailed knowledge of chemical synthesis methods and several experimental measurement techniques, including the analysis of magnetic susceptibility and neutron scattering methods. The gained knowledge is applied in pinpointing the low temperature magnetic phase transition in an ongoing project in the 
lab of two dimensional artificial magnetic (permalloy) honeycomb lattice. Two dimensional magnetic honeycomb lattice provides a unique platform to study emergent magnetic phenomena in reduced degrees of freedom. The system is expected to develop novel spin solid order at low temperature. I have performed detailed analysis of non-linear susceptibility of permalloy honeycomb lattice, which revealed the nonthermodynamic nature of phase transition to the spin solid state in this system. In the ensuing chapters, I have explained each project in great detail. A brief overview of the previous research works and the motivations behind the study is provided in the Introduction section. 


\section{Chapter 1}

\section{Introduction}

\subsection{Magnetic moment correlation and magnetic p- hase transition}

Magnets have fascinated human beings since the twilight of civilization. We can trace our interaction with them back to ancient Greece.[1, 2] We have exploited the properties of magnetism from very early days. The lodestone, a magnetic iron oxide ore, seems to have been used by the Vikings and ancient Chinese to make compass like tools for navigation. [3] Beginning of scientific investigation of magnetism can be traced back to 16th century England. From there on, numerous scientists have contributed to the understanding of the phenomena. One of the key figure that charted the path for theoretical understanding of physics of electromagnetism is James Clerk Maxwell, who showed that electricity and magnetism are part of the same fundamental force field. [4] However, the modern understanding of magnetism in terms of motion and interaction of electrons comes from the works of Ernst Ising and Werner Heisenberg. It is only after that we started understanding the fundamentals of magnetism. Since the ancient times, we have come a long way in understanding and using this very 
consequential phenomena. It has been the driving factor for modern technology and we seem ever eager to unlock its potential by understanding it in more detail.

Magnetism stems from magnetic moment of materials. Magnetic moments and their correlation play a key role in various novel properties. Magnetic moment in a material originates from unpaired spin, which is the intrinsic property of an elementary particle. The magnetic moment for a system is given by the expression,

$$
\mu_{J}=g_{J} \mu_{B} \frac{J}{\hbar}
$$

where $g_{J}$ is know as the Lande g-factor, $\mu_{B}$ is the Bohr magneton, which is $\sim 9.274$ $\times 10^{-24} \mathrm{~J} / \mathrm{T}, J$ is the total angular momentum and $\hbar$ is the Planck's constant. We try to understand the way the magnetic moments correlate and the reason behind the correlation. Several interesting phenomena, from quantum spin liquid to high temperature superconductivity, depend on the nature of correlation of magnetic moments. The correlation of magnetic moment in a system can be tuned by various external factors like heat, physical and chemical pressure, etc. In general, variation of these external parameters tend to effect the correlation of the magnetic moments. This process of change in the nature of correlation is called a magnetic phase transition. For example, if a material is a ferromagnet, the system might not show any correlation at high temperatures but the magnetic moments will align themselves in parallel configuration as the temperature is decreased below the curie temperature. A material might go through various phase transitions with respect to the external force applied to the system. Generally, interesting physics tend to appear when we take the system on the verge of criticality. It is the point where an ordered system is on the verge of becoming disordered. Various novel effects have been observed in system that have been taken to criticality. In some high temperature superconductors, the superconductivity tends to emerge when the system is taken to the verge of 
disorder from antiferromagnetically ordered state. Thus it is of utmost importance to understand the magnetic correlation and nature of magnetic phase change in a material to be able to advance their technological application.

\subsection{Static and Dynamic magnetic moment corre- lation}

The nature of correlation in magnetic moments can be explored as static and dynamic magnetic correlation. Static magnetic correlation is when the magnetic moments align with respect to each other after the critical temperature or field has been achieved. The static magnetic correlation is a time independent behavior of magnetic moments. In general terms, the static correlation is given by

$$
\left\langle S_{i}(r, t) . S_{j}\left(r^{\prime}, t\right)\right\rangle
$$

Where $S_{i}(r, t)$ and $S_{j}\left(r^{\prime}, t\right)$ are two different spins at position $\mathrm{r}$ and r' respectively.[5] This term can assume various forms that is manifested as ferromagnetic, antiferromagnetic and spin density wave systems (to name a few). The magnetic moment of a material can be paramagnetic where the moments interact very weakly with each other. The moments are not ordered in this kind of system. The system can still be magnetized by a field, however, the system does not retain its properties once the field is turned off. Similarly, we have ferromagnets, ferrimagnets and antiferomagnets. In ferromagnetic system, we have magnetic moments line up parallel to each other as we decrease the temperature or apply a certain magnetic field. In ferrimagnetic systems, we have magnetic moment line up antiparrllel to each other, however, the magnitude of the moments is different thus the system tends to have net magnetization in on direction. The anti-ferromagnetic system tends to have magnetic moments that align 
antiparallel to each other as temperature is decreased. As we apply a certain amount of field, the system tends to get rid of the antiferromagnetic correlation as the applied field will realign all the magnetic moments in the applied direction. A system in ferromagnetic or antiferromagnetic state can be explained by the hamiltonian,

$$
\widehat{H}=-\sum_{i j} J_{i j} S_{i} \cdot S_{j}
$$

where, $J_{i j}$ is the exchange interaction between two spins. If $J_{i j}>0$, then the nearest neighbor magnetic moments tend to align parallel to each other, which is the ferromagnetic state. And if $J_{i j}<0$, the magnetic moments will align antiparallel to one another. Which we call antiferromagnetic state. [5] If we want to further understand the nature of ferromagnetic and antiferromagnetic systems in presense of external magnetic field, we have to go through the Weiss model where we set up the Hamiltonian in magnetic field B as

$$
\widehat{H}=-\sum_{i j} J_{i j} S_{i} S_{j}+g \mu_{B} \sum_{j} S_{j} \cdot B
$$

Here the first term is the exchange interaction, whereas the 2nd term is the Zeeman energy. Applying the Weiss model, we consider the molecular field at $i^{\text {th }}$ site:

$$
B_{m f}=-\frac{2}{g \mu_{B}} \sum_{j} J_{i j} S_{j}
$$

from here we get the Hamiltonian to be

$$
\widehat{H}=g_{J} \mu_{B} \sum_{i} S_{i} \cdot\left(B+B_{m} f\right)
$$

Where $g_{J}$ is the Lande-g factor, $\mu_{B}$ is the Bohr magneton, B is the applied field and $\mathrm{B}_{m f}$ is the molecular field. When we treat the system as a para-magnet in magnetic 
field, we can find the temperature at which the system orders. This temperature is called the Curie temperature for ferromagnetic materials and Neel temperature for anti-ferromagnetic systems. Since, in anti-ferromagnets the two magnetic moments are opposite direction, the net magnetization is zero. We have to use what is called the Staggered Magnetization which is the net magnetization of each sublattice. This term will be a non-zero term below Neel temperature and is used for order parameters for antiferromagnets.[5]

(a)

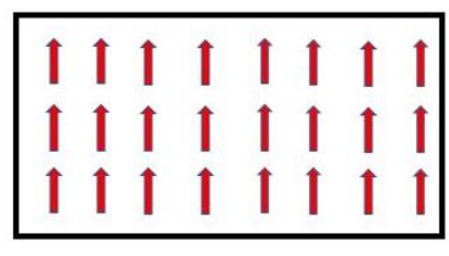

(b)

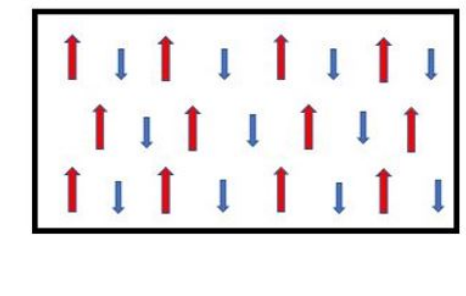

(c)

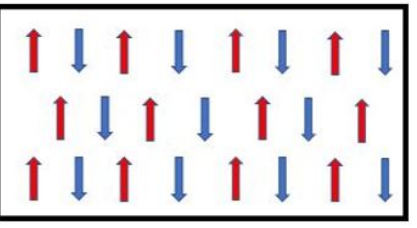

Figure 1.1: (a)Ferromagnetic ordering, where all magnetic moment align parallel to each other(b)Ferrimagnetic ordering, where magnetic moment align anti parallel to each other but the magnitude of the moment is different (c) Anti-ferromagnetic ordering, where magnetic moment align anti parallel to each other

Materials also behave as diamagnets because of the induced magnetic field opposing the applied field due to Lenz's law. When magnetic field is applied to a material with paired electrons in orbitals, the orbital motion is changed. This means that the magnetic flux is being changed. This means the system tends to avoid the change thus repelling the applied field which causes diamagnetism.[5] As explained, there are various ways a system can be statically correlated. However, there are also systems that do not have static correlation, no matter what driving force is applied. These systems are very interesting in their own light and the first two materials that we study fall in this category.

Similarly, dynamic correlation refers to the time dependent interaction of magnetic moments. The dynamic behavior of magnetic moments can shed a lot of light into 
the intrinsic magnetic behavior of the system. This is why it is an important avenue to pursue if we want to understand the novel properties in materials. To better understand the relationship between the novel phenomena and the static and dynamic correlation of magnetic moments, we have performed detailed study in materials which show no static ordering but show a plethora of interesting dynamic behavior.

\subsection{Monosilicides - Interesting case of Nickel Mo- nosilicide}

Several silicides have been studied previously as they are neighbors to pnictides and chalcogenides that show superconducting properties. Various silicides such as $\mathrm{V}_{8} \mathrm{Si}$, $\mathrm{MoSi}_{0.7}$, etc show superconducting behavior.[6] Other silicides with magnetic ion that have 3d configuration i.e. $\mathrm{Mn}, \mathrm{Fe}, \mathrm{Ni}, \mathrm{Cr}$, etc have been studied to explore the novel magnetism like skyrmion lattice $(\mathrm{MnSi}) .[7,8,9,10]$ While a lot of the silicides have been studied in great detail and have their magnetic properties explored, NiSi remains an exception. NiSi, with FeAs-type orthorhombic crystal structure, is widely used in microelectronics industry to create contact for field-effect transistor or the nanoelectronic devices.[11, 12] Both electron and hole carriers, deriving from large Fermi surfaces, participate in the electrical conduction in NiSi.[13, 14] Despite the enormous technological significance of NiSi metal, its magnetic properties are largely unexplored due to the apparent non-magnetic ground state.[14]

It has also been observed in previous research that the $\mathrm{NiSi}$ is a compensated metal where electron and hole carriers in equal numbers. It has been observed that above $40 \mathrm{~K}$, the system tends to have electrons as the majority charge carriers. As we decrease the temperature from 40K, holes become the dominant charge carriers. [13] 


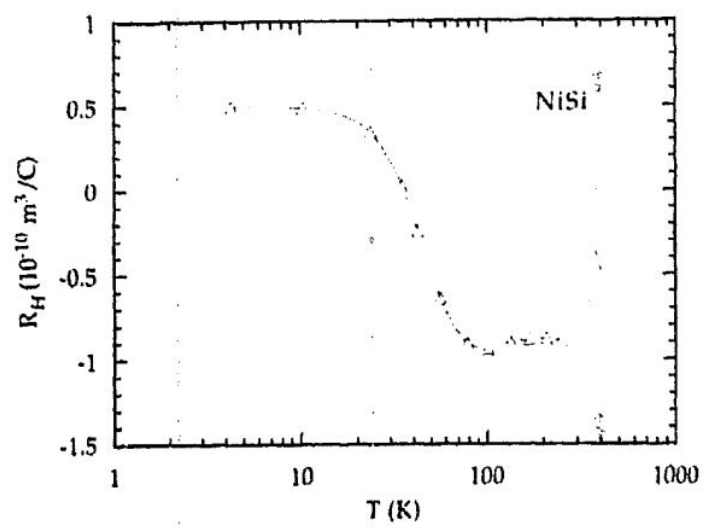

Figure 1.2: Hall measurement of $\mathrm{NiSi}$, where we see a change in the nature of charge carriers at $\sim 40 \mathrm{~K}$. Reprinted with permission from B. Meyer et al. [13]. Copyright 2019 by the Elsevier.

Detailed investigation of magnetic properties in poly-crystalline NiSi using the complementary techniques of ac susceptibility, electrical, neutron scattering and dc susceptibility measurements reveal unexpected new properties that are never observed before. One of the key observation is the presence of anomalous frequency dependent diamagnetic signal. This system shows very peculiar property where dynamic correlation, which shows one-to-one correspondence to the superconducting transition, despite the absence of static ordering. Random phase approximation (RPA) explains this dynamic behavior in NiSi, where the magnetic Ni atoms are assumed to be randomly oriented and interact isotropically with their nearest neighbor only. By using this approximation, which is given by

$$
\chi^{\prime \prime}(Q, \omega)=\Sigma_{ \pm} \frac{\omega \chi_{0} \Gamma_{Q^{ \pm}}}{\Gamma_{Q^{ \pm}}^{2}+\omega^{2}}
$$

where

$$
\Gamma_{Q^{ \pm}}=\Gamma\left[1 \mp \chi_{0} J_{1}\right]
$$

we get the information about the strength of the interaction given by $J_{1}$, the time dependence of the interaction $\Gamma_{Q}$ and the length of the correlation $\chi_{0}$. In this method, 
electrons are assumed to respond to an external perturbing potential which oscillates with single frequency $\omega$. The contribution to the perturbation from total electron potential is averaged out and only the potential from wave vector $\vec{k}$ contributes. [15]

\subsection{Presence or absence of magnetic correlation in ruthenates}

Chemically doping $\mathrm{CaRuO}_{3}$ with $\mathrm{Co}$ to create artificial disorder tends to show similar behavior where the static ordering is missing but the system shows dynamic correlation which have one to one correspondence with interesting physical and electrical property. The parent compound $\mathrm{CaRuO}_{3}$ is itself a metallic perovskite that is paramagnetic in nature.[16] $\mathrm{CaRuO}_{3}$ is also shown to have two non-fermi liquid states.[17] The system exhibits strong magnetic fluctuations.[18] It has also been observed that the material has cooperative quantum spin fluctuations.[19] 


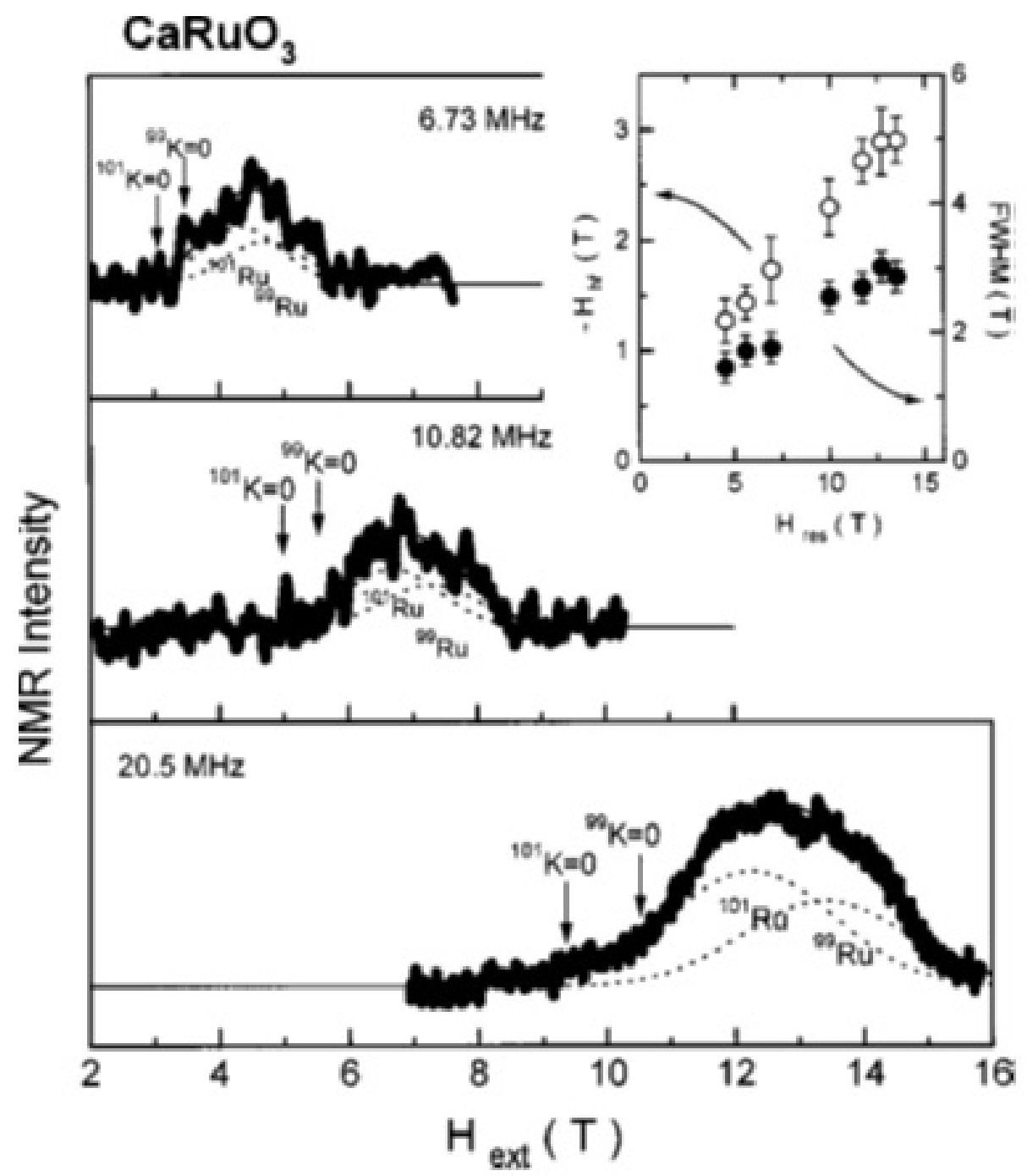

Figure 1.3: Ru-NMR spectra at different frequencies taken at $4.2 \mathrm{~K}$. The magnitude of $H_{h f}$ and FWHM increases as $H_{\text {res }}$ increases. This study shows that there are strong magnetic fluctuations present in the system. Reprinted with permission from H. Mukunda et al. [18]. Copyright 2019 by the American Physical Society.

The quantum spin fluctuation is key to the understanding of the microscopic mechanism behind the novel quantum critical phenomenon or the quantum spin liquid state.[20, 21, 22] The quantum spin liquid state arises from the phase coherent quantum fluctuation of entangled spin-1/2 with net spin of 0 and $1 \mu B$. [23] Depending on the nature of the entanglement, whether it is an ordered partitioning of nearest neighbor spins or the superposition of an infinite partitioning of nearest as well as 
further away spins that are simultaneously pointing along different directions, the collective ensemble of dimers can be characterized as the valence bond solid (VBS) or the valence bond liquid (VBL), respectively.[24, 25, 26] Since the moment is in continuous quantum fluctuation mode, the singlet state is either excited to the triplet state or the valence bond breaks and a continuum excitation due to the fluctuation of individual spin yields a liquid-like gapless state.[24, 27] An inherent frustration in the disorder free triangular lattice plays an important role in the realization of these novel effects.

However, creating a disorder free magnetic lattice is a difficult task to achieve.[28, 29] Taking an altogether different route, we have explored the quantum mechanical properties of spin-1/2 in a disorder induced tailored frustrated system. We create the disorder by doping the parent compound $\mathrm{CaRuO}_{3}$ with $\mathrm{Co}$ ions. A quenched disorder prohibits the propagation of long-range static or dynamic order, hence automatically creates frustration. We have studied in detail the nature of dynamic magnetic correlation in Co doped $\mathrm{CaRuO}_{3}$. The dynamic correlation at low temperature of Co doped $\mathrm{CaRuO}_{3}$ are explained by a singlet-to-triplet excitations of spins that involve nearest and next nearest neighbor interaction. To get this relation, we used the equation

$$
I(Q)=|F(Q)|^{2} \Sigma m_{i}^{2}\left[1-\sin \left(Q d_{i} /\left(Q d_{i}\right)\right)\right]
$$

where $m_{i}^{2}$ is the squared moment per formula unit and $d_{i}$ is the distance between nearest and next nearest neighbor. [30, 31, 19] As the temperature is increased, the system tends to continuum of excitation involving interaction beyond nearest neighbor. The details of the experiments and analysis for $\mathrm{Co}$ doped $\mathrm{CaRuO}_{3}$ are presented in chapter 3 . 


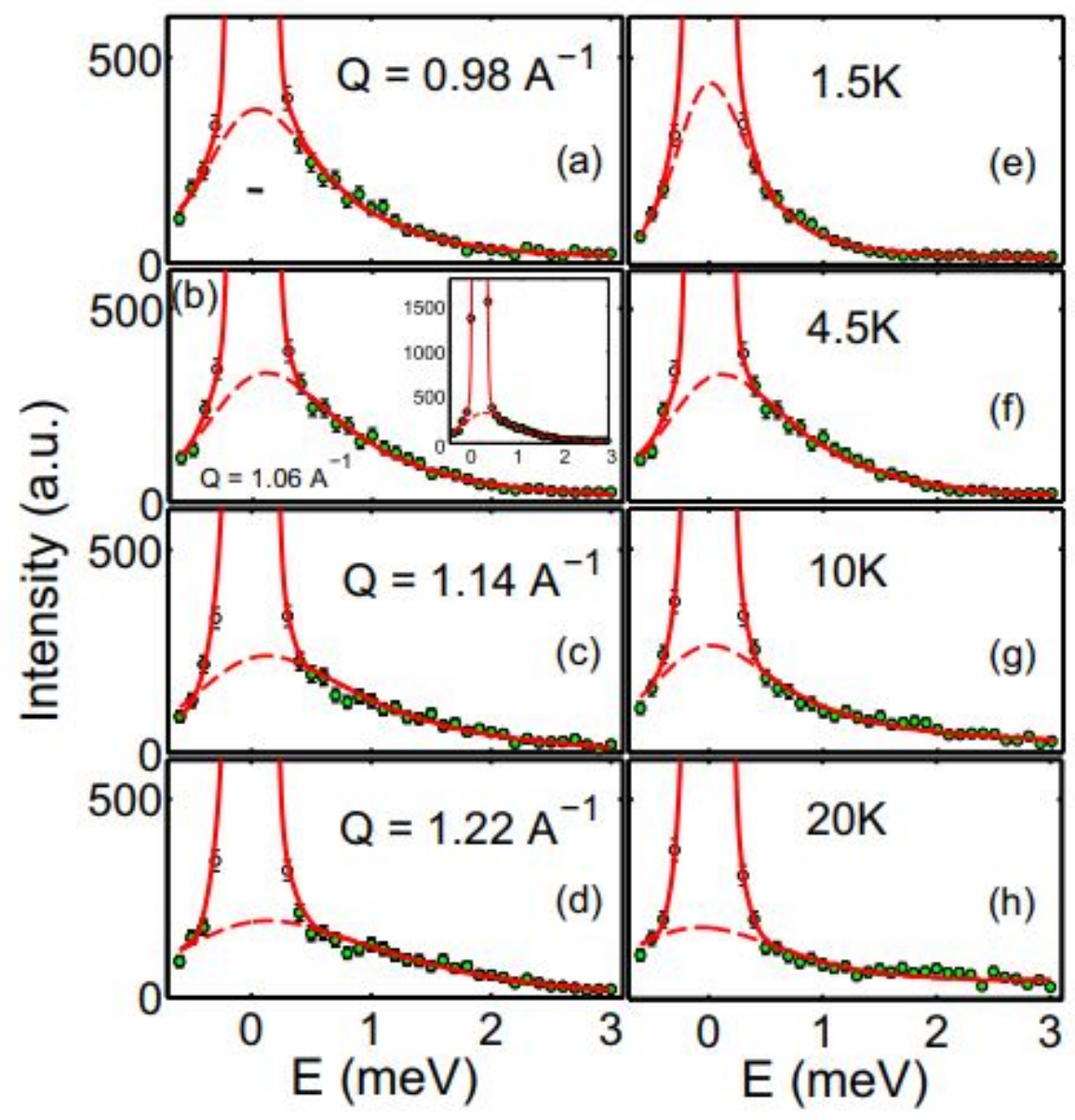

Figure 1.4: Inelastic neutron scattering measurements, carried out on SPINS for $\mathrm{CaRuO}_{3}$, as a function of energy. (a)(d) Characteristic energy scans at constant $\mathrm{Q}$ at $\mathrm{T}=4.5 \mathrm{~K}$ are plotted in these figures. The solid line represents the complete fitting profile of the background corrected experimental data due to paramagnetic fluctuation model, convoluted with the instrument resolution and consists of an elastic line (Gaussian) and a quasielastic function multiplied by the detailed balance factor. The quasielastic data (dashed lines) are well described by a Lorentzian-squared line shape, emphasizing the cooperative paramagnetic nature of Ru4+ spins fluctuation in the system. As the absolute $Q$ increases, the peaks become broader and weaker, suggesting a magnetic nature of fluctuation. (e)(h) Energy scans at constant temperatures at $\mathrm{Q}=1.06 \AA^{-1}$ are plotted. The inelastic spectra become stronger as the measurement temperature is reduced. The error bar in all figures represents one standard deviation. 


\subsection{Static magnetic correlation in $\mathrm{EuMn}_{2} \mathrm{As}_{2}$}

We have explored $\mathrm{EuMn}_{2} \mathrm{As}_{2}$ which is a 122-pnictide magnet where strong static correlation is present. Several such pnictides have previously been studied to understand the magnetism and its interplay with superconductivity. Many pnictide materials, such as $\mathrm{BaFe}_{2} \mathrm{As}_{2}, \mathrm{EuFe}_{2} \mathrm{As}_{2}$, have shown unconventional superconductivity where the underlying magnetism is argued to play an important role in the Cooper pair formation[32, 33, 34, 35, 36, 37]. Magnetism and superconductivity often seem to compete, however, in some materials these two states seem to coexist.[38, 39] Magnetism and superconductivity also seem to have interplay in various strongly correlated materials. [40] Thus we know that electronic interactions in strongly correlated system tend to alter the physical and magnetic properties and give rise to several phases of matter. [41, 42, 43]

In addition to the interplay between magnetism and superconductivity, pnictides are also at the forefront of the exploration of magnetism, such as valence fluctuation and itinerant magnetism $[44,45,46,47,48,46]$. Many of these compounds manifest

intriguing physical and magnetic properties due to the competing interplay between $4 \mathrm{f}$ and $3 \mathrm{~d}$ magnetism. Often enough, these materials exhibit multiple magnetic-ordered regimes that are intricately related to the underlying lattice structure[49] . Thus, exploring the magnetic ordering in the system can provide important insight into the properties of the material. The other reason to study $\mathrm{EuMn}_{2} \mathrm{As}_{2}$ is that it is an anomaly in the family of 122-pnictides. While most of the 122-pnictides crystallize in tetragonal structure, this compound tends to crystallize in trigonal structure.[50] 


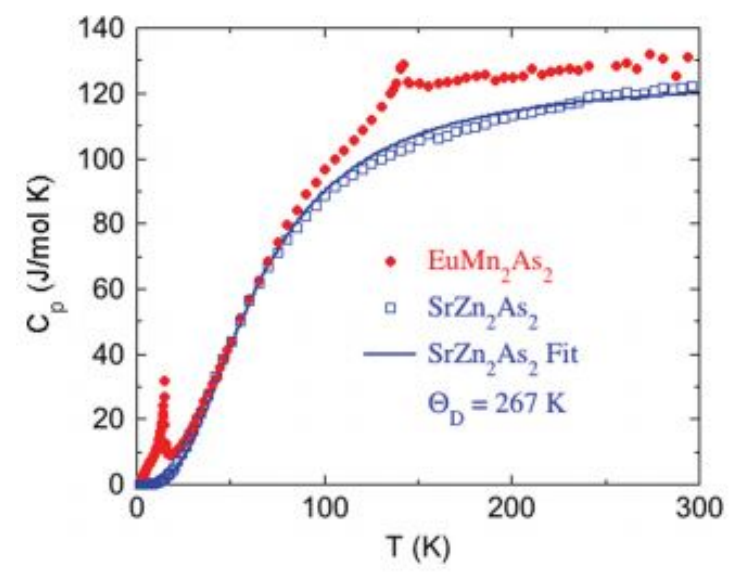

Figure 1.5: Heat Capacity measurement data for $\mathrm{EuMn}_{2} \mathrm{As}_{2}$ shown by the red dots. Two transitions at $\sim 14 \mathrm{~K}$ and $142 \mathrm{~K}$ are observed. Reprinted with permission from V.K. Anand et. al [51]. Copyright 2019 by the American Physical Society.

Previously electrical, magnetic and heat capacity measurements have been performed where two magnetic transition has been reported [51], however, the correlation of magnetic moments is not well understood. We have performed detailed neutron scattering measurement to understand this correlation. In chapter 4, I go through the details of the study.

\subsection{Magnetic phase transition in two-dimensional permalloy honeycomb lattice}

After studying the static and dynamic correlation in bulk materials, we have used several experimental techinques such as magnetic susceptibility measurements to explore the nature of magnetic phase transition in 2D system. We have done non-linear susceptibility scaling analysis to explore how the phase change occurs in artificial honeycomb lattice. Honeycomb lattice structures have generated significant research interest in recent times, primarily motivated by unusual electronic and magnetic properties, as found in graphene, silicene, $\mathrm{MoS}_{2}$, and artificial magnetic honeycomb lattices, respectively.[52, 53, 54, 55] 
Artificial magnetic honeycomb lattices, initially conceived to explore the statistical properties of spin ice systems in two-dimension,[56] have raised many distinct issues and developed into their own field of study. At the core of them lies the local moment arrangements of two-in and one-out (or vice-versa) or all-in or all-out configurations on a given vertex of the lattice that gives rise to a highly degenerate ground state.[55] Here, two-in and one-out refer to two moments, aligned along the length of the honeycomb element, one points to the vertex and the other points away from the vertex. Similarly, the all-in configuration indicates that all moments are pointing to the vertex of the lattice as shown in Fig. 5.1. The ease in tunability of the structure allows us to study a plethora of magnetic phenomena, such as spin ice, spin liquid, and topological magnetic properties, in a disorder free environment.[57]

The system is explained by the Hamiltonian given by,

$$
H=-J \sum_{\langle i j\rangle} \sigma_{i} \sigma_{j}\left(\widehat{e}_{i} \cdot \widehat{e}_{j}\right)+\frac{D r_{n n}^{3}}{2} \sum_{i \neq j} \sigma_{i} \sigma_{j} \frac{\left(\widehat{e}_{i} \cdot \widehat{e}_{j}\right)-3\left(\widehat{e}_{i} \cdot \widehat{r}_{i j}\right)\left(\widehat{e}_{j} \cdot \widehat{r}_{i j}\right)}{\left|r_{i}-r_{j}\right|^{3}}
$$

The first term is the exchange interaction where $J$ is the strength of the interaction whereas $D=\left(\frac{\mu_{0}}{4 \pi}\right) \frac{\mu^{2}}{r_{n n}^{3}}$ is the characteristic strength of dipolar coupling. [58]

Recent theoretical calculations predict the presence of a rich phase diagram of emergent spin correlated phases in an artificial magnetic honeycomb lattice that would be difficult to achieve in a naturally occurring bulk material. It includes the temperature dependent evolution of spin ice order to the spin liquid state, followed by an entropy-driven magnetic charge-ordered state as a function of reducing temperature. $[55,57]$ At much lower temperature, the system is predicted to develop a spin solid order, consisting of alternating arrangements of vortex loops of opposite chiralities.[59,60] Since the vortex magnetization profile implies zero net magnetic moment, the spin order in a honeycomb lattice is expected to exhibit zero magnetization and entropy state. The transition to this novel spin solid state is theoretically 
predicted to be thermodynamic. However, after doing detailed analysis on the magnetic susceptibility measurement, we have observed that the transition to spin solid state is not thermodynamic in this system. We present details on this analysis in chapter 5 .

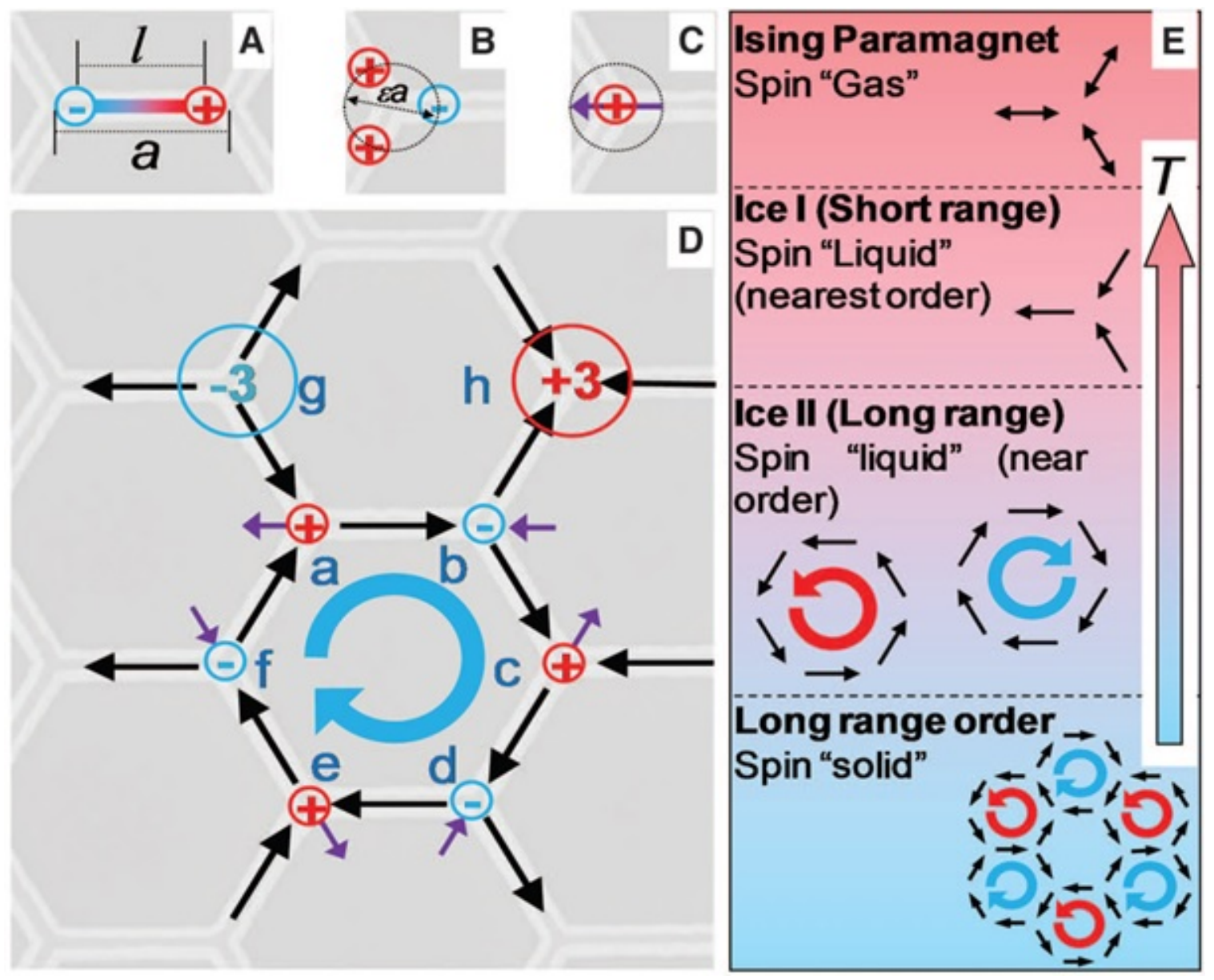

Figure 1.6: (A) a single bar element as a dumbbell, (B) the uneven charge distribution around the vertex, $(\mathrm{C})$ the vertex dipole moment, and (D) the possible vertex configurations. The right side (E) shows the four distinct phases that are predicted to be accessible in the system as temperature decreases. Reprinted with permission from Branford et al. [57]. Copyright 2019 by the The American Association for the Advancement of Science. 


\section{Chapter 2}

\section{Study of Magnetism in NiSi}

Discovery of superconductivity in iron pnictides and chalcogenides (eg. FeSe, NaFeAs, LiFeAs etc.) have generated a lot of research in the exploration of superconductivity in nonmetal-based solid-state materials $[61,62,63]$. The experimental investigations, connecting magnetic ion vacancies to the underlying magnetism and the superconducting state, in alkali-metal intercalated iron selenides provide a new perspective to the current understanding [64]. As silicides are close neighbor to the pnictides and chalcogenides, they provide a plethora of possibility to explore new and interesting physics.For example, monosilicides $\mathrm{MSi}$, where $\mathrm{M}$ is a magnetic ion with $3 \mathrm{~d}$ electronic configuration ( $\mathrm{Mn}, \mathrm{Fe}, \mathrm{Ni}, \mathrm{Cr}$, etc.), provide a rich avenue to study novel magnetism of fundamental importance, such as skyrmion lattice $(\mathrm{MnSi})$, unconventional magnetic insulator $(\mathrm{FeSi})$, and spin singlet state $(\mathrm{CoSi})[7,8,9,10]$. In many cases, however, the noncentrosymmetric cubic structure plays key role. In addition to the novelty in the fundamental properties, some of these materials are also used extensively for technological applications [65]. For instance, NiSi, with FeAs-type orthorhombic crystal structure, is widely used in the microelectronics industry to create contact for the field effect transistor or the nanoelectronics devices [11, 12]. Both electron and hole carriers, deriving from large Fermi surfaces ${ }_{16}$ participate in the electrical conduction in 
NiSi $[13,14]$. Despite the enormous technological significance of NiSi metal, its magnetic properties are largely unexplored due to the apparent nonmagnetic ground state [66]. Detailed investigation of the magnetic properties in polycrystalline NiSi using the complementary techniques of ac susceptibility, electrical, neutron scattering, and dc susceptibility measurements reveals unexpected new properties. We show that NiSi exhibits a minority superconducting phase, which coexists with the majority metallic phase below $T<100 K$.

\subsection{Sample Fabrication}

The polycrystalline samples of NiSi were synthesized by conventional solid-state reaction method using ultrapure ingredients of Ni (99.996 purity) and Si (99.999 purity) powders. Starting materials were mixed in stoichiometric composition, pelletized and sintered at $970^{\circ} \mathrm{C}$ for $24 \mathrm{~h}$ in a sealed quartz tube, and evacuated to the pressure of $10^{-4}$ torr. The sample was cooled slowly to room temperature in another $8 \mathrm{~h}$. The sample obtained was shiny metallic pellet as shown in Fig. 2.1

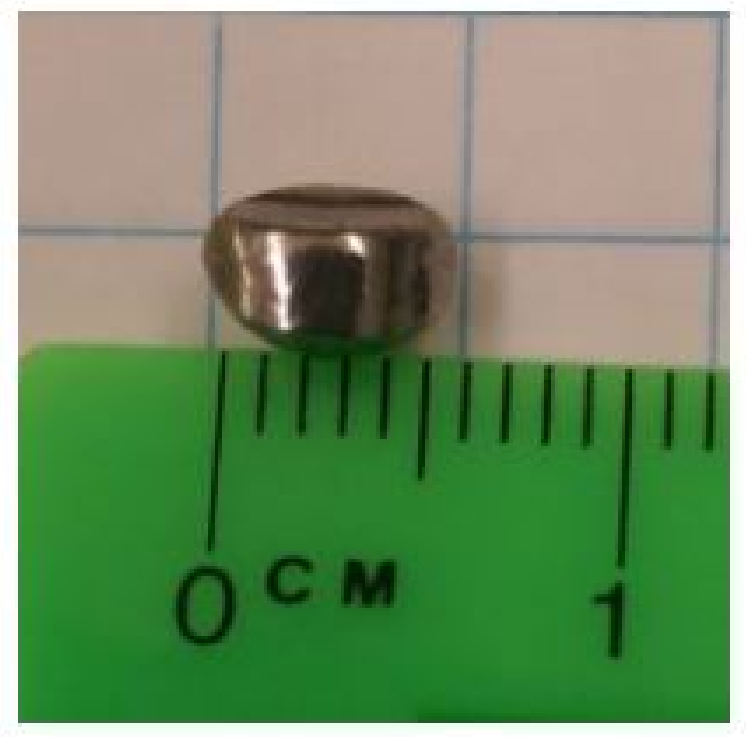

Figure 2.1: Sample obtained after sintering under vacuum. 


\subsection{Structural Characterization}

X-Ray Diffraction measurements were performed using Simens D500 Diffractometer, that used copper target with $K_{\alpha}$ with wavelength $1.54 \AA$. X-Ray diffraction is the elastic scattering of X-Ray photons by atoms in a periodic lattice. During the scattering, the X-Ray that are in phase get constructively interfered whereas the ones that are out of phase destructively interfere. One can derive the diffraction of X-rays by crystal planes using Bragg's law. Which is:

$$
n \lambda=2 d \sin \theta
$$

where $\mathrm{n}$ is an integer, $\lambda$ is the wavelength of the $\mathrm{X}$-ray, $\mathrm{d}$ is the characteristic spacing between the crystal planes of given sample and $\theta$ is the angle between the incident beam and the normal to the reflecting lattice plane. We can measure the angles, $\theta$, under which the constructively interfering X-rays leave the crystal. From this, the inter planar spacing, d, can be determined.

The shiny sample exhibited pure NiSi phase. The x-ray diffraction (XRD) data was refined using the Rietveld powder diffraction refinement. As shown in Fig.3.1, all peaks in the x-ray diffraction pattern fit to the NiSi phase, thus confirming the single phase of the sample. The refined structure was identified with the Pnma orthorhombic

structure of NiSi, with lattice parameters of $\mathrm{a}=5.186 \AA$, $\mathrm{b}=3.331 \AA$, and $\mathrm{c}=5.625 \AA$, also consistent with other reports on the synthesis of high-quality samples of NiSi $[13]$. 


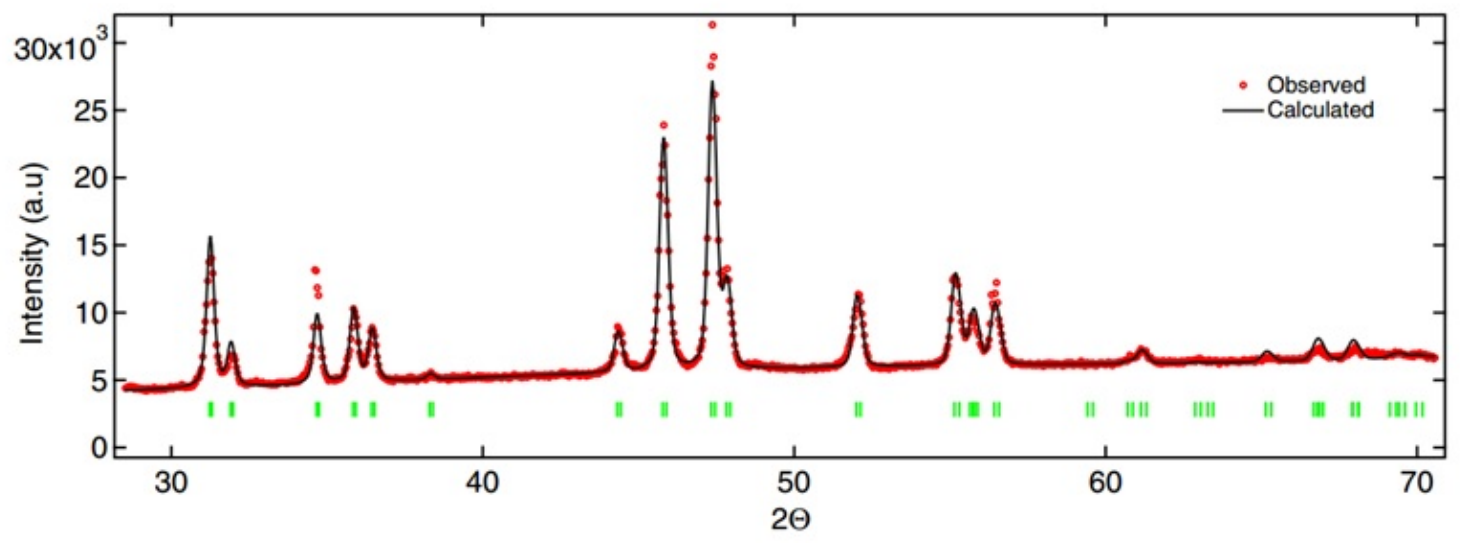

Figure 2.2: X-ray diffraction pattern of NiSi. Powder x-ray diffraction spectra of NiSi powder used in this study. The powder diffraction data is refined using FULLPROF suite for Rietveld analysis. The high purity of the sample is evident from the XRD data, where every single peak is identified to the orthorhombic structure (crystallographic group Pnma) of NiSi.

\subsection{Heat Capacity Measurements}

Heat capacity measurement was performed using Quantum Design Physical Property Measurement System(QD PPMS). The QD PPMS uses Two Tau method to get the heat capacity of the system. This method removes any error due to the contact between the platform and the sample, as shown in Fig. 2.3. A simple model, assumes good thermal contact between the sample and the sample platform whereas in Two Tau model it considers a poor thermal contact. In a simple model, the temperature $\mathrm{T}$ of the platform as a function of time obeys the equation:

$$
C_{\text {total }} \frac{d T}{d t}=-K_{w}\left(T-T_{b}\right)+P(t)
$$

where $C_{\text {total }}$ is the total heat capacity of the sample and sample platform; $K_{w}$ is the thermal conductance of the supporting wires; $T_{b}$ is the temperature of the thermal bath or the sample puck frame; and $P(t)$ is the power applied by the heater. The heater power $P(t)$ is equal to $P_{0}$ during the heating portion of the measurement and equal to zero during the cooling portion. The solution of this equation is given by 
the exponential function with time constant $\tau$ equal to $C_{\text {total }} / K[67]$.

In Two-Tau model, the system considers poor thermal contact between sample and platform. The model simulates the effect of heat flowing between the sample platform and sample and the effect of heat flowing between the sample platform and puck. It is expressed as:

$$
\begin{gathered}
C_{\text {platform }} \frac{d T_{p}}{d t}=P(t)-K_{w}\left(T_{p}(t)-T_{b}\right)+K_{g}\left(T_{s}(t)-T_{p}(t)\right) \\
C_{\text {sample }} \frac{d T_{s}}{d t}=-K_{g}\left(T_{s}(t)-T_{p}(t)\right)
\end{gathered}
$$

where $C_{\text {platform }}$ is the heat capacity of the sample platform, $C_{\text {sample }}$ is the heat capacity of the sample, and $K_{g}$ is the thermal conductance between the two due to the grease. $T_{p}(t)$ and $T_{s}(t)$ are the temperatures of platform and sample respectively[67].

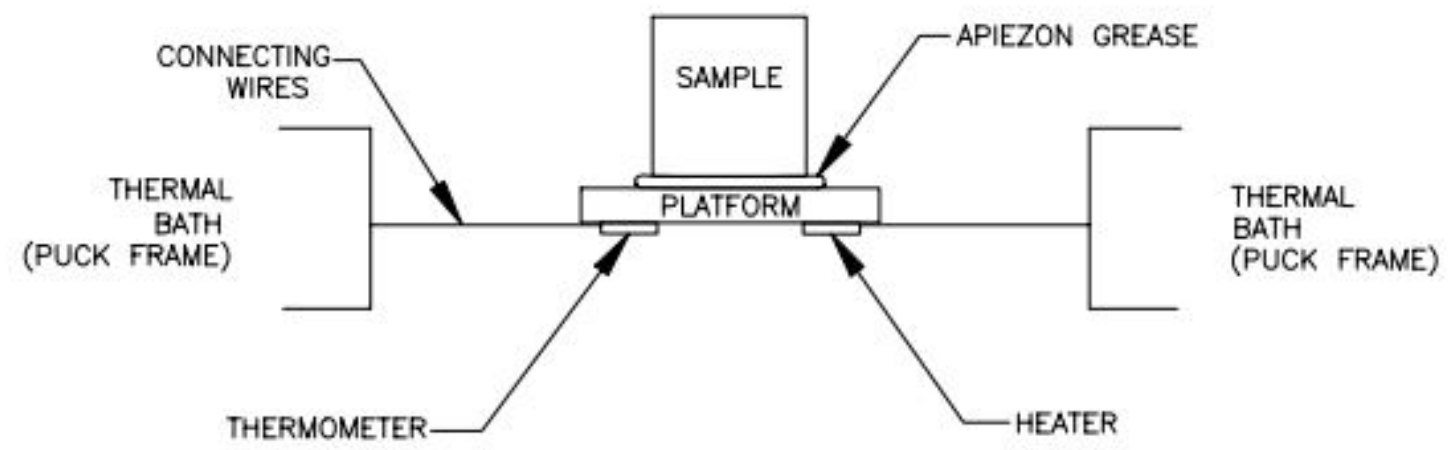

Figure 2.3: Figure shows the schematics of Heat Capacity measurement (Figure taken from QD PPMS heat capacity manual)

Heat capacity measurement gives information about the phonon and electron contribution to the heat capacity. It is given by the equation:

$$
C_{\text {metal }}={ }_{20} \gamma T+\beta T^{3}
$$


Where, $\gamma$ gives the information about the electron contribution and $\beta$ gives information about the phonon contribution. Phonon refers to the quantized lattice vibrations. No transition was observed in the heat capacity measurement performed in NiSi sample. The values for $\gamma=1.6 \mathrm{~mJ} \mathrm{~mol}^{-1} \mathrm{~K}^{-2}$ and $\beta=0.031 \mathrm{~mJ} \mathrm{~mol}^{-1} \mathrm{~K}^{-4}$ are close to the values of $\gamma=1.73 \mathrm{~mJ} \mathrm{~mol}^{-1} \mathrm{~K}^{-2}$ and $\beta=0.0317 \mathrm{~mJ} \mathrm{~mol}^{-1} \mathrm{~K}^{-4}$, reported in previous literature.[13] As seen on Fig. 2.4, the metallic behavior dominates the heat capacity.

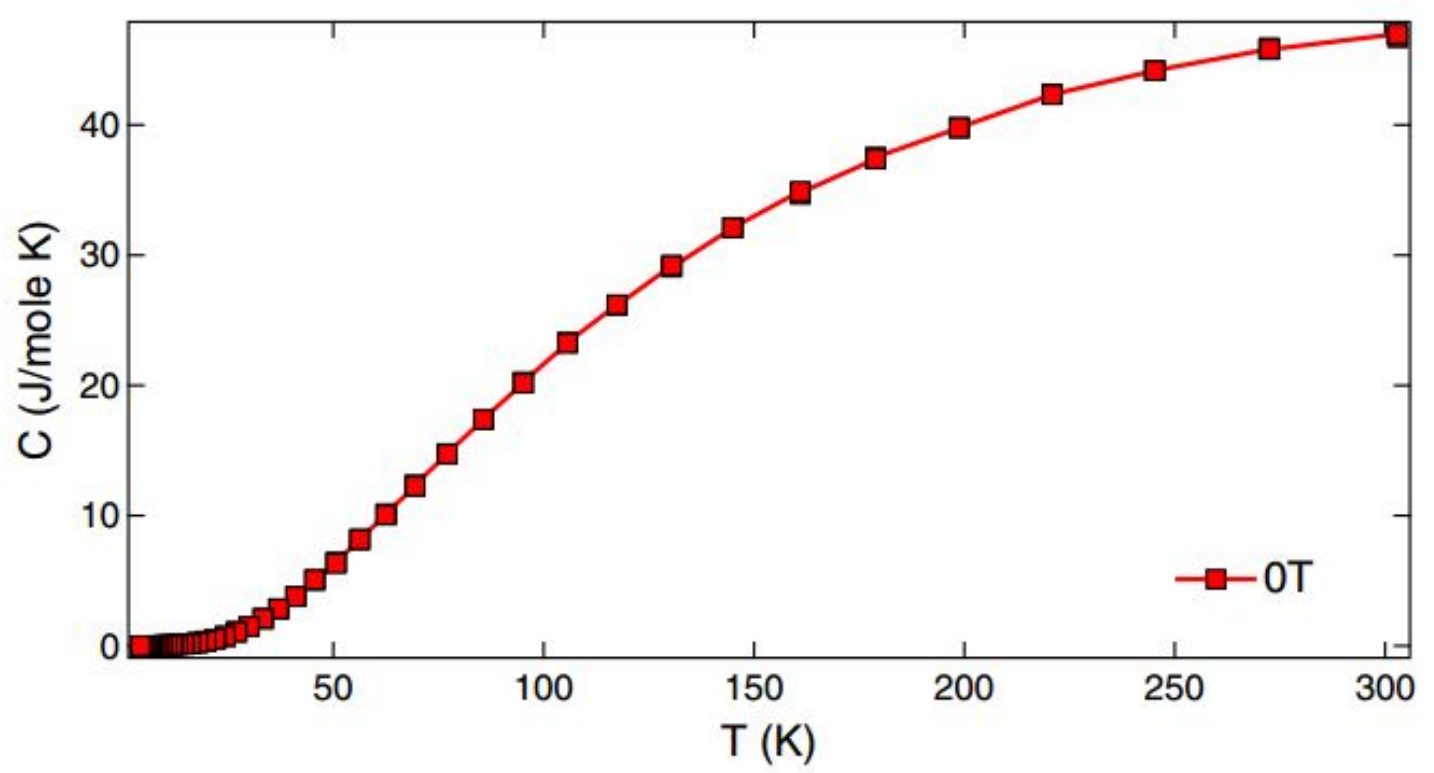

Figure 2.4: Figure shows heat capacity of NiSi taken using QD PPMS 


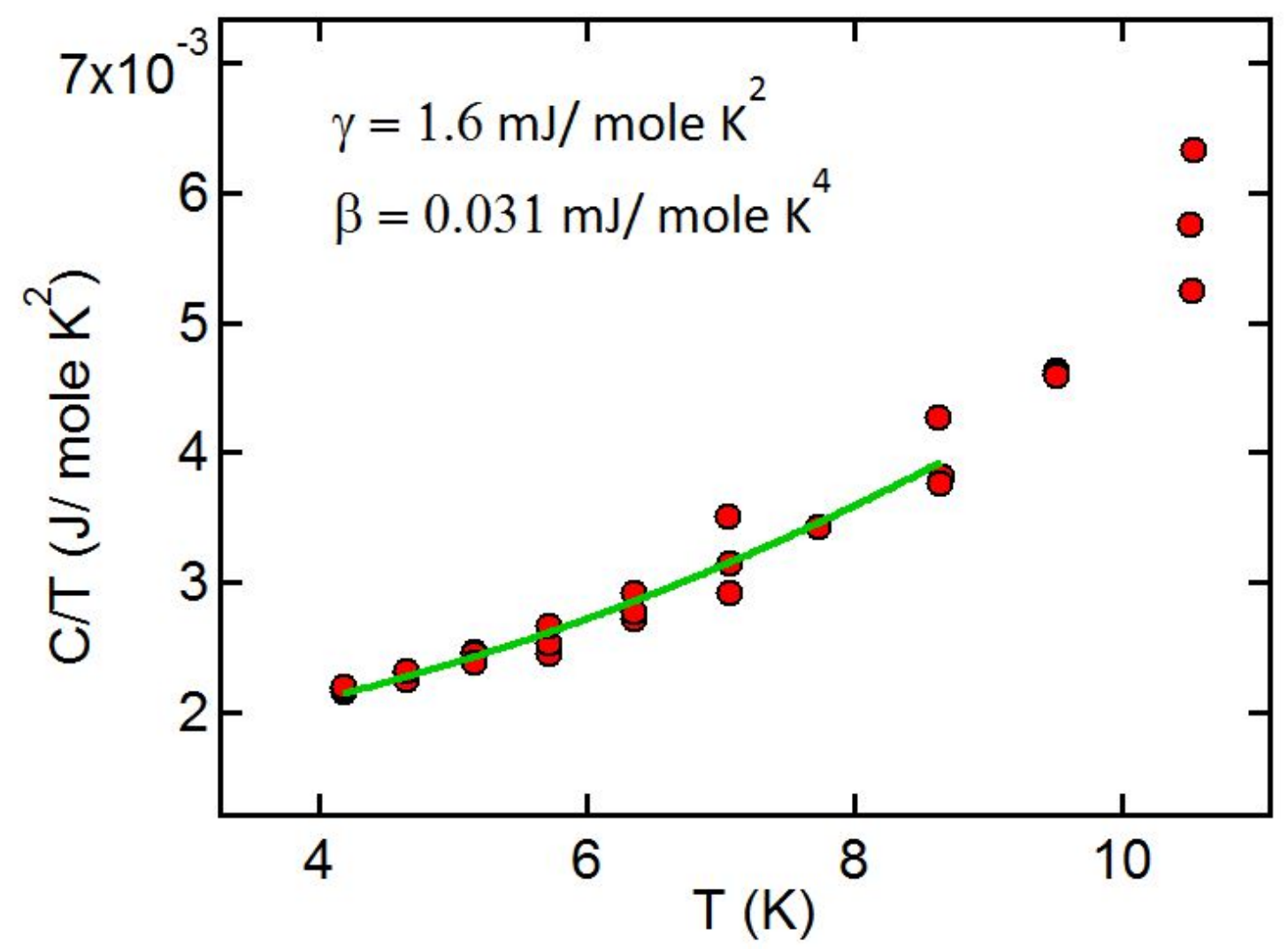

Figure 2.5: Figure shows the fitting done on heat capacity data taken at low temperatures. The data was fitted with equation $C / T=\gamma+\beta T^{2}$, the value of $\gamma$ and $\beta$ are similar to the ones observed in previous literature. [13] Note: the three points per temperature is because of the nature of the measurement. The system takes three sets of measurements around the selected temperature. 


\subsection{Electrical Measurements}

A four-probe technique was employed to measure the electrical resistance of NiSi. For the four-probe measurements, equidistant contacts were made on a $5 \mathrm{~mm}$ size sample. The measurement done on the sample showed metallic behavior upto low temperature of $2 \mathrm{~K}$ with very low resistivity. However, a small kink was observed around $\sim 75 \mathrm{~K}$ in the resistivity data at $0 \mathrm{~T}$. The kink type feature disappeared as measurement was performed by applying magnetic field. In Fig. 2.7 we show the resistivity data at 0 T. We observe metallic behavior with very small resistivity whereas a small cusp like feature is observed around $\sim 75 \mathrm{~K}$. In Fig. 2.8 we show resistivity vs temperature at several fields. We observe that the cusp like feature disappears with the application of field. Thus we think there is some kind of transition that is magnetic in nature around this temperature.

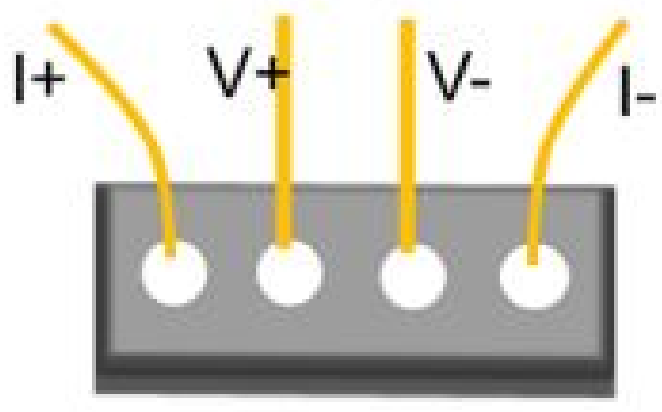

Figure 2.6: Figure shows the schematics of 4 Probe measurement. I is the current applied at the outer leads and $\mathrm{V}$ is the voltage measured at inner leads. 


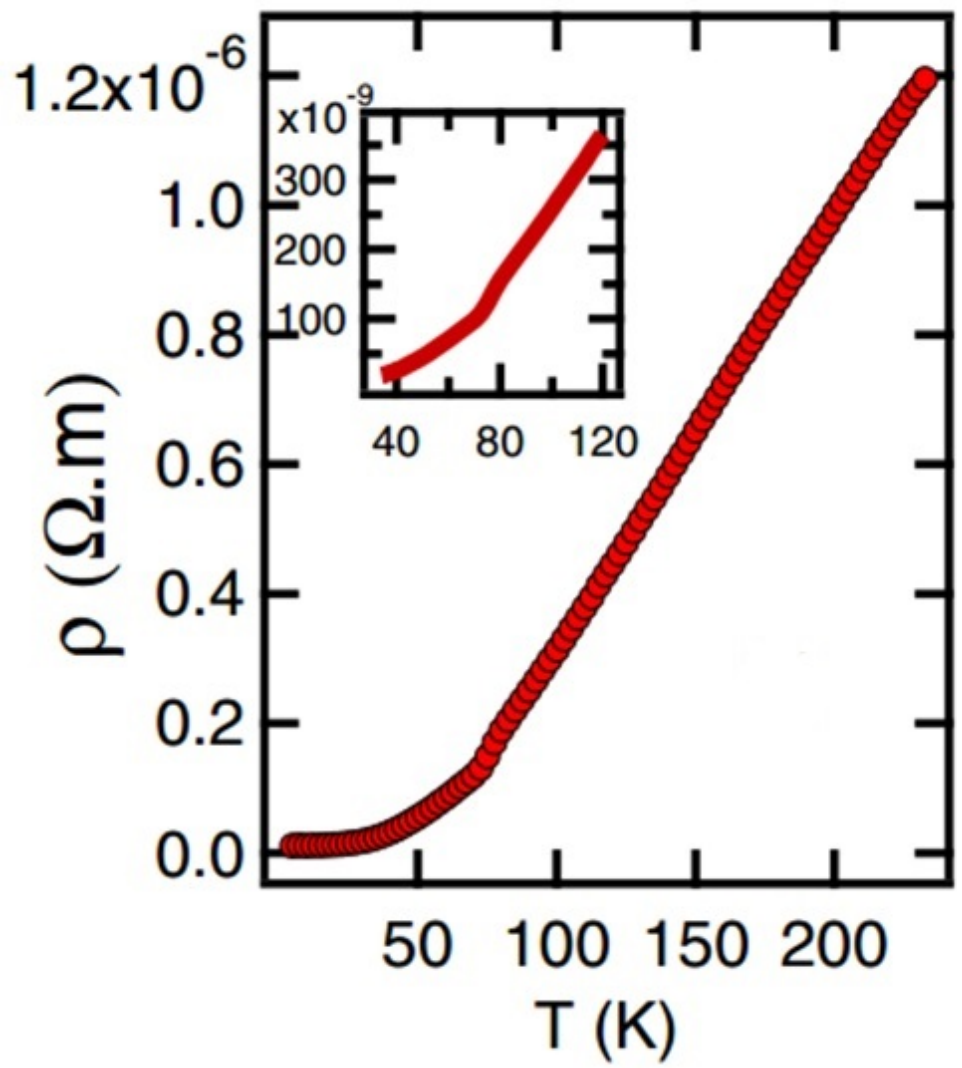

Figure 2.7: Very low electrical resistivity, consistent with previous reports on a highquality sample of NiSi, is detected across the entire measurement range of electrical resistivity. The inset highlights a small sharp drop in resistivity at $T \sim 75 \mathrm{~K}$. 


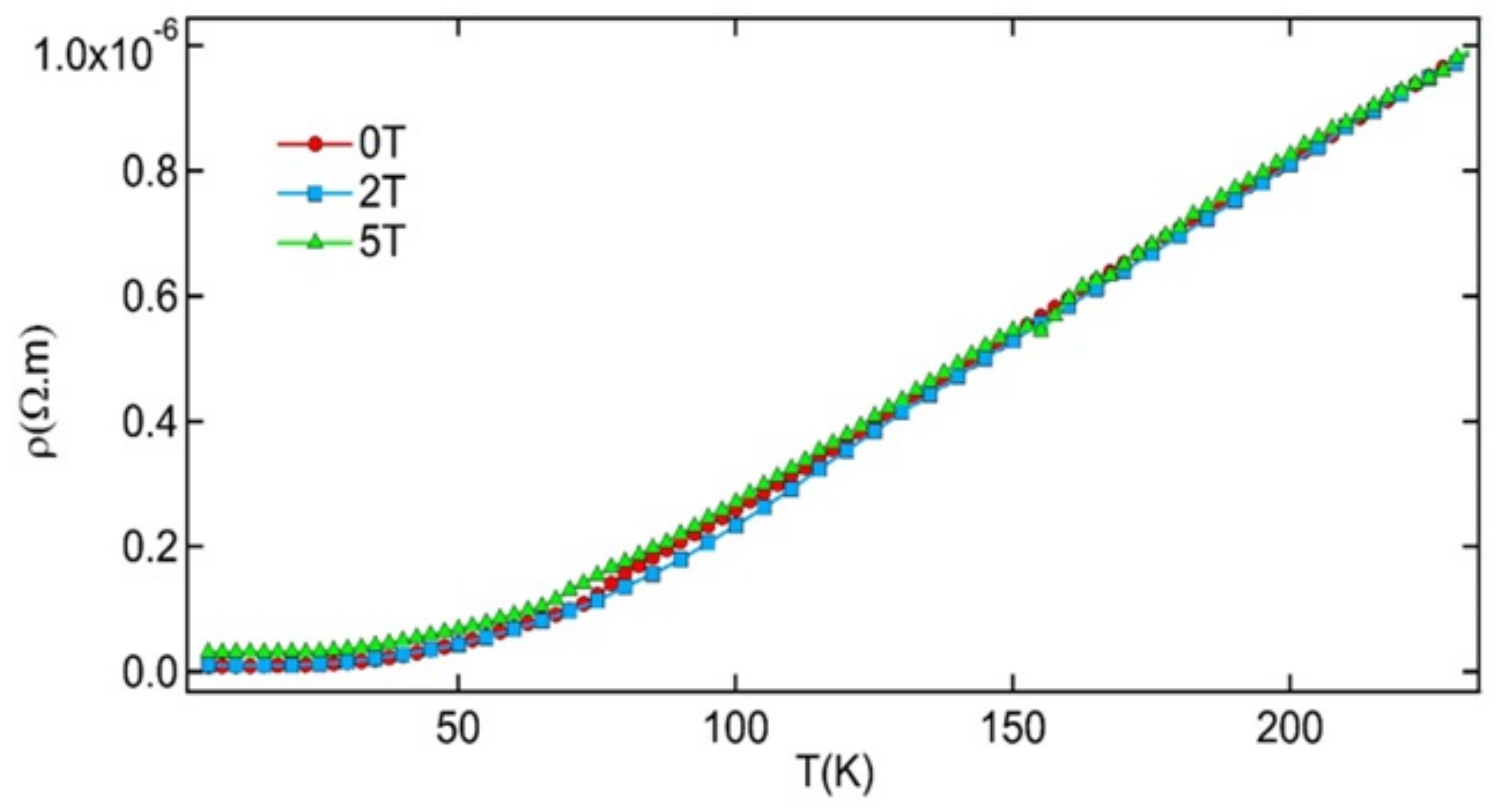

Figure 2.8: The sharp drop observed at $0 \mathrm{~T}$ data disappears as field is applied. 


\subsection{Magnetization Measurements}

\section{AC Susceptibility Measurements}

AC Magnetization measurements were done using Quantum Design Physical Property Measurement System(QD PPMS). The AC measurements are done by applying a small AC field on top of a DC field. This causes a time-dependent moment in the sample. The field of the moment then induces a current in the pick-up coils of the QD PPMS and the measurement is made. At low frequency of the AC field the magnetization curve of the material is very similar to that of a DC measurement. When the AC field is small, the induced AC moment is

$$
M_{A C}=(d M / d H) \cdot H_{A C} \sin (\omega t)
$$

where $H_{A C}$ is the amplitude of the driving field, $\omega$ is the driving frequency and $\chi=d M / d H$ is the slope of $M(H)$ curve.

At higher frequencies, the AC magnetization is different than the DC magnetization curve. This is due to time dependent dynamic effects in the sample. In AC measurements with higher frequencies, the magnetization of the sample may lag behind the applied magnetic field. The AC susceptibility measurement therefore has two quantities: the magnitude of the susceptibility $\chi$ and the phase shift, $\varphi$. The $\varphi$ is considered relative to the applied AC signal. The AC susceptibility can be considered as having a real in-phase component $\chi^{\prime}$ and out-of-phase imaginary component $\chi$ ". These can be represented as

$$
\chi^{\prime}=\chi \cos \varphi
$$

and

$$
\chi "=\chi \sin \varphi
$$

where $\chi=\sqrt{\chi^{\prime 2}+\chi^{\prime \prime 2}}$ and $\varphi=\underset{26}{\arctan }\left(\chi^{\prime \prime} / \chi^{\prime}\right)$. The $\chi^{\prime}$ gives the magnetic suscep- 
tibility of the sample whereas the $\chi$ " gives information about the dissipative process in the sample.

To further understand the magnetic property, both static and dynamic, we study the AC susceptibility of the system. Quantum Design Physical Properties Measurement System with a temperature range of $2-300 K$ was used to perform AC susceptibility measurements. AC susceptibility data was taken as a function of temperature for the ac frequency range of $10-10^{4} \mathrm{~Hz}$. Measurements were done on applied magnetic field of 10 Oe and 1 Oe.

The net ac susceptibility $\chi(T)$ is written as $\chi(T)=\chi^{\prime}(T)+i \chi^{\prime \prime}(T)$, where the real part $\left(\chi^{\prime}\right)$ represents static magnetic behavior and the imaginary part $\left(\chi^{\prime \prime}\right)$ provides information about the energy loss due to damping in a system. It is immediately noticed that as the temperature is reduced, both static $\left(\chi^{\prime}\right)$ and dynamic $\left(\chi^{\prime \prime}\right)$ susceptibilities develop highly unusual frequency dependencies below $T \sim 100 K$. While $\chi^{\prime}$ becomes more negative and tends to saturate at low temperature, $\chi$ " becomes more positive and tends to form a peak-type structure at low temperature. This is more evident in Fig. 2.10, where the measurements of ac susceptibilities in 1 Oe ac magnetic field exhibiting sharp peaks in $\chi "$ are observed. Such frequency dependence of ac susceptibility is the hallmark of a superconductor $[66,68]$. This type of behavior has been observed in samples with pronounced granularity. The mechanism for the behavior is explained by the grains becoming superconducting first and the shielding to the applied magnetic filed being provided by the intra grain currents. As the grains and the sample as a whole are at different states, the damping is enhanced at the critical temperature. The damping reduces as the temperature is decreased as more and more grains become superconducting. On further decreasing the temperature, the sample as a whole becomes superconducting, thus the damping becomes smaller.[66] 


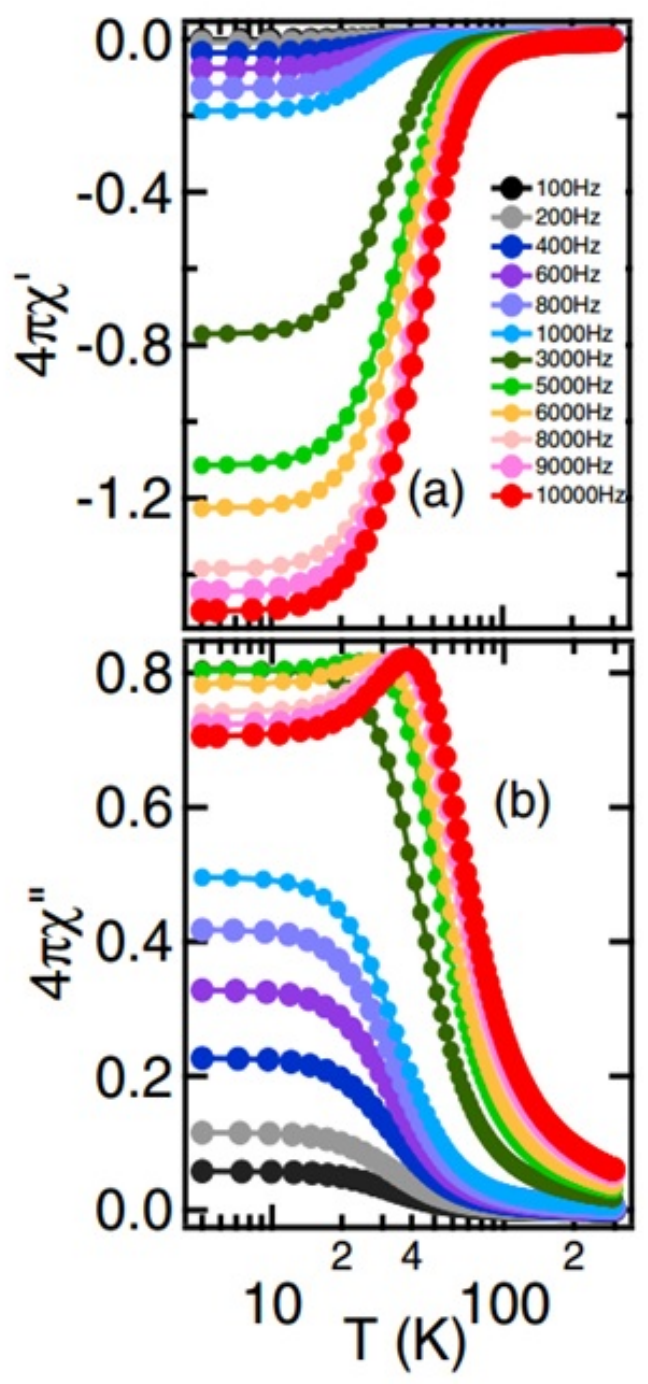

Figure 2.9: (a) and (b) The ac susceptibility measurement as a function of temperature of NiSi.The ac frequency varies from 10 to $10_{4} \mathrm{~Hz}$. NiSi exhibits diamagnetic behavior throughout the measurement temperature range. In a remarkable observation, the static susceptibility $\chi^{\prime}$ is found to develop frequency dependence below $T \sim 100$ $\mathrm{K}$. The diamagnetic response becomes stronger as ac frequency increases. For the same measurement range of temperature and frequency, bulk dynamic susceptibility $\chi$ " exhibits a peak-type positive response. The peak-type feature moves to higher temperature and becomes stronger as the ac frequency increases. A superconductor usually exhibits these behaviors. 


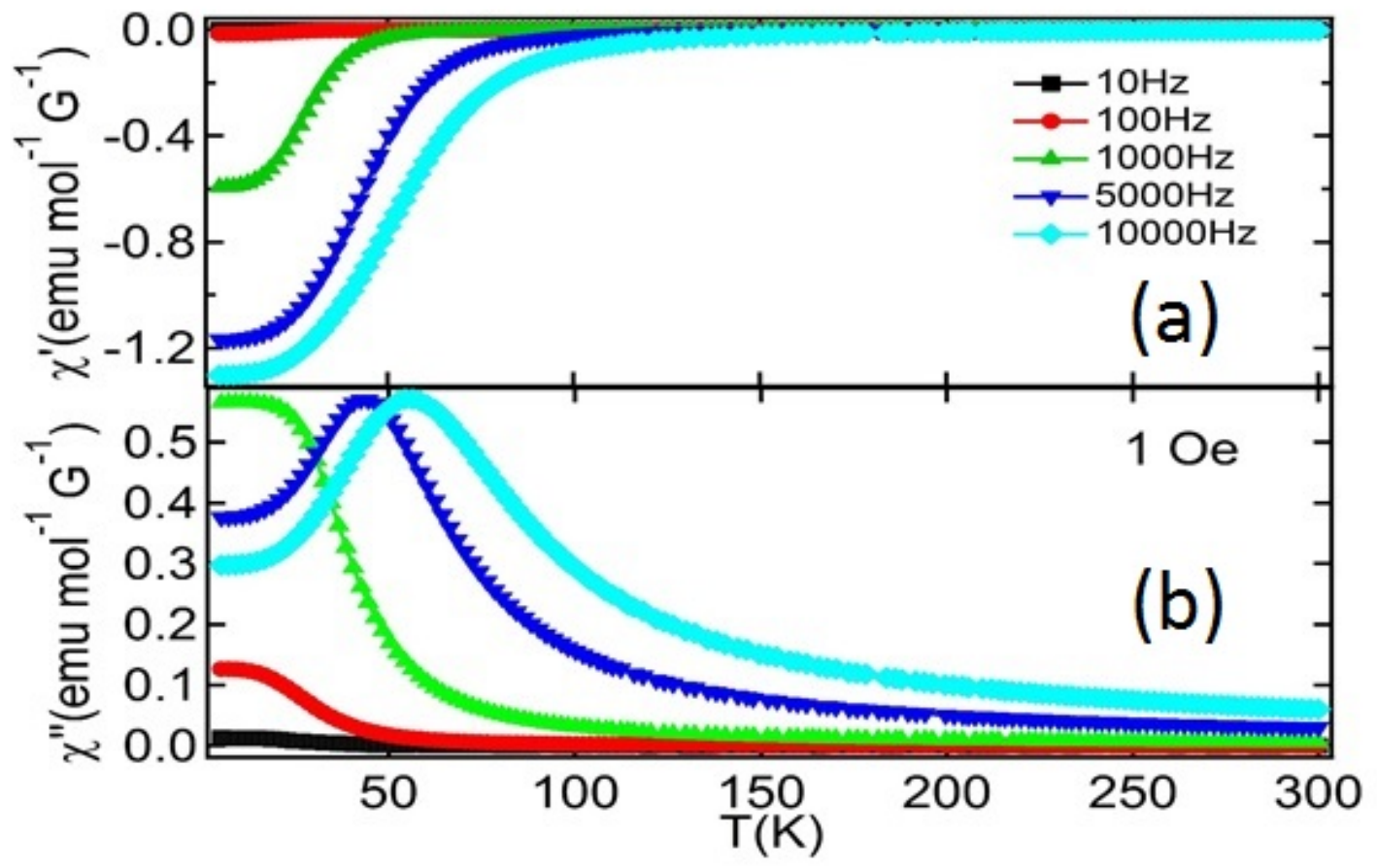

Figure 2.10: (a) and (b) The ac susceptibility measurement as a function of temperature of NiSi at 1 Oe.The ac frequency varies from 10 to $10^{4} \mathrm{~Hz}$. The peak at bulk dynamic susceptibility $\chi "$ is enhanced as the applied field is decreased. 


\section{Susceptibility Measurements}

One of the hallmark of a superconductor is the Meissner Effect. It is expressed as perfect diamagnetism in magnetic measurements. Meissner effect is responsible for the shielding of applied magnetic field in a superconducting sample. Whenever a sample is placed in an externally applied magnetic field, it will induce a current. The magnetic field produced by this induced current will oppose the applied magnetic field. This is explained by the Lenze's law. In a normal metallic sample, the induced current along with the induced field will dissipate due to the resistance of the sample. However, in a superconductor, the induced current does not dissipate as the resistance is zero. This allows the induced current to flow infinitely and thus repel the applied magnetic field, giving rise to the diamagnetism observed in superconductors. To test this effect, we performed DC magnetization measurements in our sample.

Quantum Design SQUID magnetometer with temperature range of $2 \mathrm{~K}-300 \mathrm{~K}$ was used to perform DC susceptibility measurements. The diamagnetic nature of NiSi was confirmed by DC magnetization measurements. In addition to the diamagnetic behavior, a peak-type feature at $T \simeq 55 \mathrm{~K}$ is observed in $\mathrm{DC}$ susceptibility. The peak is followed by the strong enhancement in the diamagnetic signal in both the field cooled (FC) and the zero field cooled (ZFC) curves. FC and ZFC curves exhibit different responses to the diamagnetic enhancement and are thus highly irreversible. Moreover, the strength of the diamagnetic signal becomes weaker as the applied field is increased. In general, a superconducting system exhibits a similar diamagnetic response to magnetic field application. However, the susceptibility of NiSi at $T=5$ $\mathrm{K}$ is much smaller compared to the perfect diamagnetic response of a superconductor, i.e., $4 \pi \chi=1$. Which means that the superconducting phase is in very small amount in our sample. 


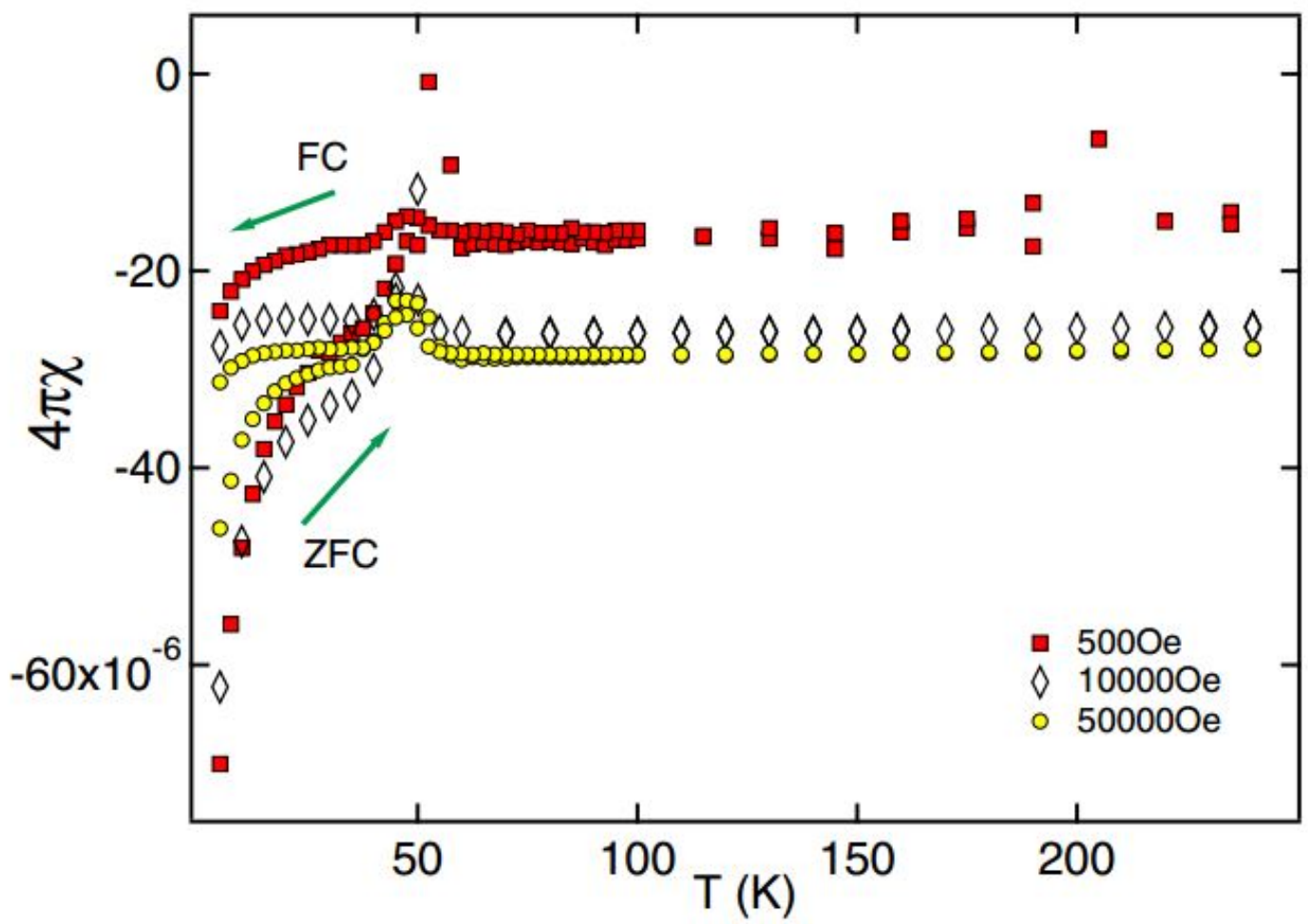

Figure 2.11: DC susceptibility measurements of NiSi. Characteristic plots of dc susceptibility as a function of temperature at a few different applied magnetic field values. The diamagnetic nature of nickel silicide is confirmed using dc susceptibility measurements. In addition to that, two distinct features are observed in dc susceptibility data that are generally observed in a superconductor: strong enhancement in the diamagnetic values of ZFC (Zero Field Cooled) and FC (Field Cooled) below $T \simeq 55 \mathrm{~K}$, and irreversibility in the $\mathrm{ZFC}$ and $\mathrm{FC}$ responses to field application. It is also noticed that as the applied field strength increases, the irreversibility becomes weaker. 


\subsubsection{Test for Normal Skin Effect}

First we consider the most common mechanism which could be giving rise to the peculiar frequency and temperature dependence in the bulk $\mathrm{NiSi}$, the normal skin effect in a metal. Skin effect refers to the behavior of conductors in AC current or field. When an AC current is applied to a conductor, the magnetic field around the axis of the conductor produces a variation in the current density. The current tends to flow more densely on the outermost part. This effect is more stronger for higher frequency. Similarly, as we apply AC field is applied on the sample, we will observe the same effect. As the AC field is applied, it will induce alternating current in the sample, this will in turn induce magnetic field that opposes the applied field. Since the effect is frequency dependent, we observe this as frequency dependent diamagnetism.

The effect is most pronounced when the skin depth, $\delta$, is maximum, or, when $\delta \sim R$ for a finite sample of size R[69]. It results in maximum damping loss, hence the $\chi$ " becomes maximum. The corresponding $\chi^{\prime}$, given by

$$
4 \pi \chi^{\prime}=(\delta / 2 R) \tanh (2 R / \delta)-1
$$

where:

$$
\delta=\frac{c}{2 \pi} \sqrt{\frac{\rho}{\mu f}}
$$

Here, $\mathrm{R}$ is the grain size, $\delta$ is the skin depth, $\mu$ being the permeability of $\mathrm{NiSi}, \mathrm{f}$ is the frequency, and $\rho$ is the resistivity. At the maximum skin depth becomes negligible. This is opposite to our observations in 2.9(a) and 2.9(b). In NiSi, both quantities $\chi^{\prime}$ and $\chi$ " maximize at the same frequency. Also, the skin effect resistance, derived from $\chi^{\prime}$, does not match the experimental data as shown in 2.12 . 


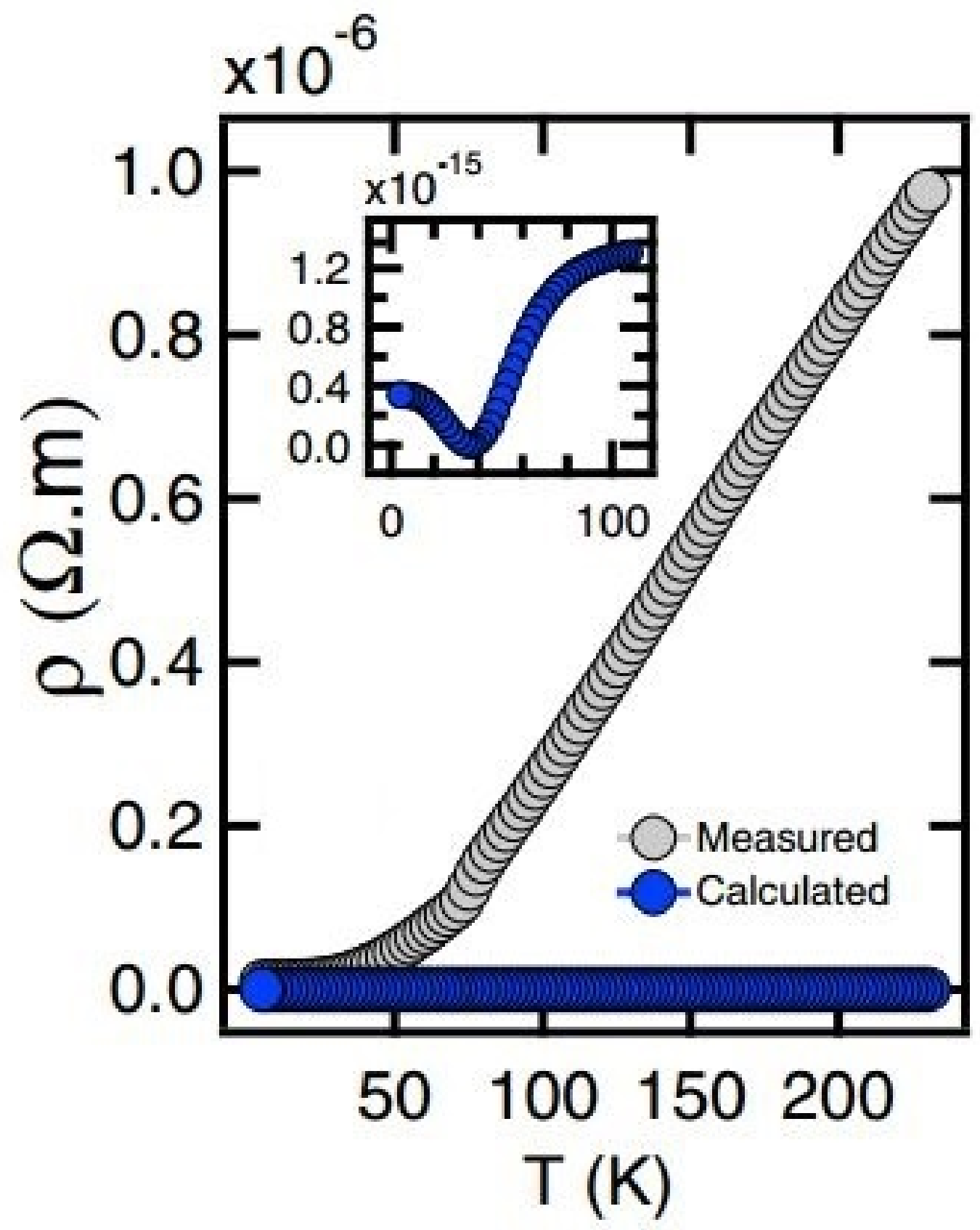

Figure 2.12: Electrical resistance due to the normal skin effect, as derived from the static susceptibility at $\gamma=10 \mathrm{KHz}$, is plotted with experimental resistance data in light gray. Clearly, the experimental data is not described by the normal skin effect, thus ruling out this possibility. 
An alternative scenario involves the intergranular interaction between penetrating field flux. Although this behavior is much more pronounced in a polycrystalline high temperature superconductor, it can also be applicable in a metal as long as the ac measurement frequency is very low and the penetrating flux in different grains of the sample behave as if the thermally activated vortex jumps between favorable metastable states of the vortex lattice (in a high temperature superconductor). The electrodynamics of a high- $\mathrm{T}_{c}$ superconductor is same as the electrodynamics of a normal metal in thermally activated flux flow regime, i.e., where the Ohms law is still valid. Such a scenario leads to a distinct loss in $\chi "$ as well as an enhancement in the diamagnetic $\chi^{\prime}$. Under this formulation, the quantities $\chi^{\prime}$ and $\chi^{\prime \prime}$ are given by [70]

$$
\chi^{\prime}=\left(\frac{\omega}{\left(\pi \mu_{0} H\right)} \int_{0}^{2 \pi / \omega}\langle B\rangle \cos (\omega t) d t\right)-1
$$

and

$$
\chi^{\prime \prime}=\left(\frac{\omega}{\left(\pi \mu_{0} H\right)} \int_{0}^{2 \pi / \omega}\langle B\rangle \sin (\omega t) d t\right)
$$

Where, $\omega$ is the frequency of the ac field, $\mu_{0}$ is the permeability of vacuum, $\mathrm{H}$ is the applied field, $\mathrm{B}$ is the resistance. The calculated plots for $\chi^{\prime}$ and $\chi$ ", using the experimental electrical resistance of NiSi, are shown in Fig. 2.13. While the dynamic susceptibility $\chi "$ is found to increase with the applied ac frequency, the static susceptibility remains negligible. This is very different from the experimental observations. 


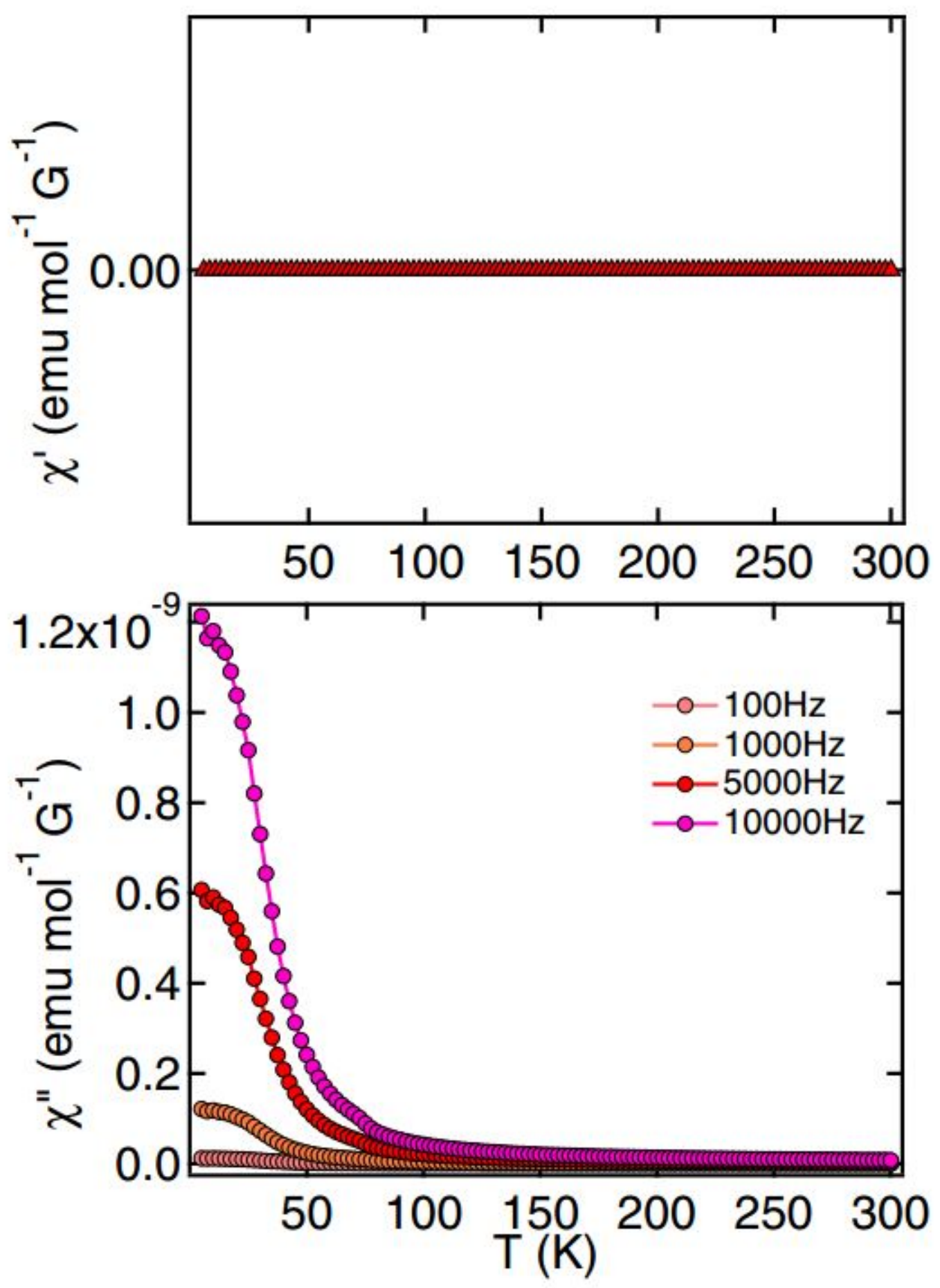

Figure 2.13: Plots of $\chi^{\prime}$ and $\chi^{\prime \prime}$ as deduced from the electrical resistance of NiSi. The calculated ac susceptibilities for a few different ac frequencies using the inter granular interaction between the penetrating flux in different grains. Clearly, it does not explain the experimental data. 
Measurements were also performed on NiSi, mixed with nonmagnetic insulator boron carbide, to further test the presence of normal skin effect. For this purpose, NiSi powder was mixed with a small amount, $15 \%$, of insulating and nonmagnetic $B_{4} C_{3}$ powder and measured both the ac susceptibility and the electrical resistance. While the sample was still highly conducting, the diamagnetism completely disappears, see Fig. 2.14. If it was indeed the normal skin effect, the frequency-dependent response should have persisted, as the high conductivity due to the flow of current in the skin of the material is still preserved. These results further rule out the normal skin effect in the system.

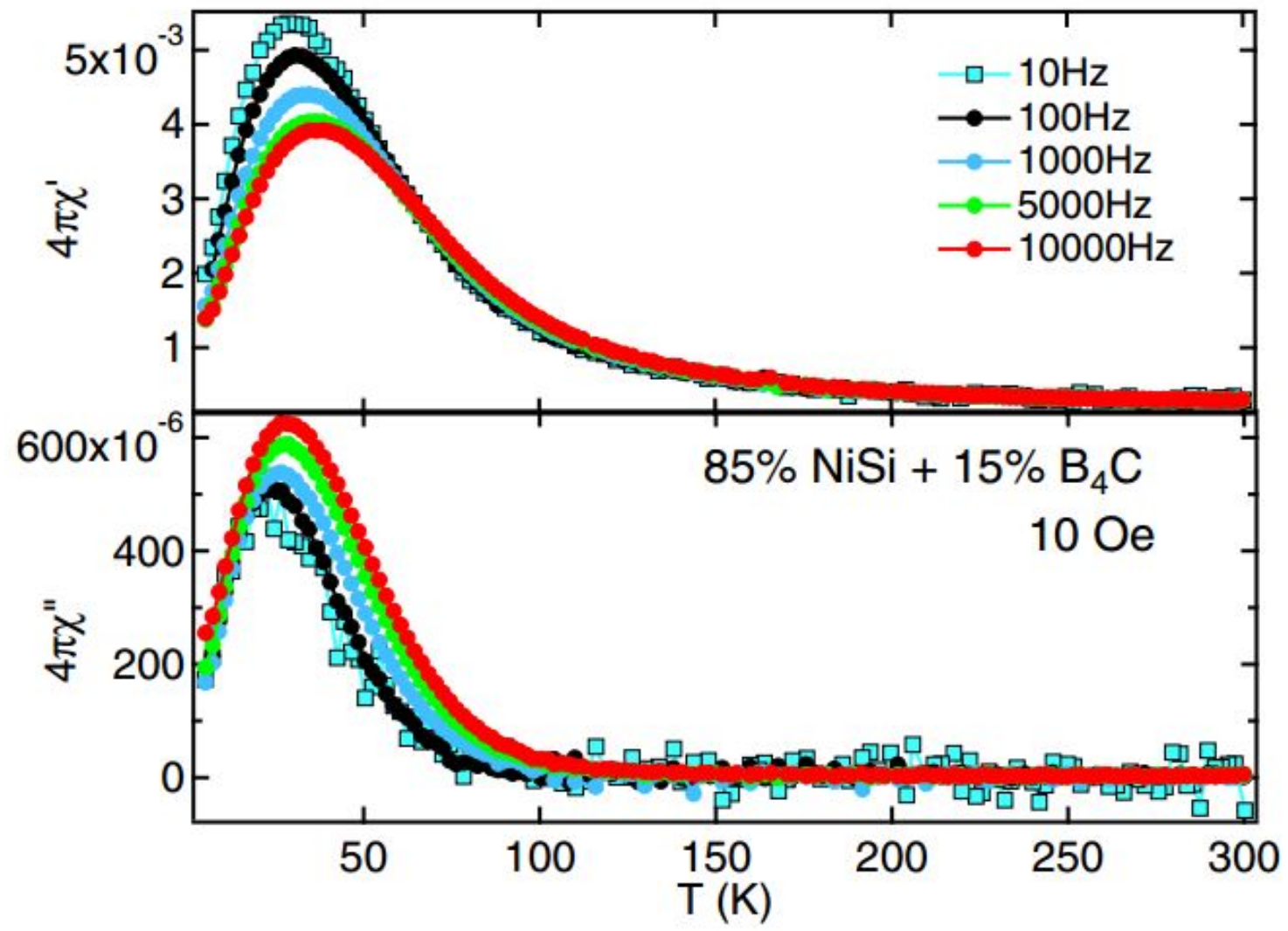

Figure 2.14: AC susceptibility measurements of NiSi mixed with $B_{4} C_{3}$ powder. The ac excitation field was $\mathrm{H}_{a c}=10$ Oe. NiSi powder was mixed with a small amount $(\simeq 15 \%)$ of insulating and nonmagnetic $B_{4} C_{3}$ powder to test the presence of skin effect. As shown here, the diamagnetism completely disappears (a) even though the sample was highly conducting, thus ruling out skin effect in the system. 


\subsubsection{Neutron Scattering Measurement}

Neutrons interact with matter through short range nuclear force and dipole dipole interaction with unpaired electrons. Since the neutron does not have any electric charge, neutrons can penetrate deep into the sample. As neutrons have magnetic moments, they interact with the magnetic moment of the sample. Because of these characteristics, neutron scattering is an excellent technique to probe the static and dynamic magnetic properties in detail.

In neutron scattering measurements, we are measuring the number of neutrons scattered per second into a particular solid angle at particular energy. As seen in Fig. 2.15 :

$$
\begin{gathered}
\Phi=\text { number of incident neutrons per } \mathrm{cm}^{2} \text { per second } \\
\sigma=\text { total number of neutrons scattered per second } / \Phi \\
\frac{d \sigma}{d \Omega}=\frac{\text { number of neutrons scattered per second into } d \Omega}{\Phi d \Omega} \\
\frac{d^{2} \sigma}{d \Omega d E}=\frac{\text { number of neutrons scattered per second into } d \Omega \text { and } d E}{\Phi d \Omega d E}
\end{gathered}
$$

We perform two different kind of neutron scattering measurement. Elastic neutron scattering is where the final energy of the neutrons is the same as the initial energy. In inelastic measurement the final energy of the neutrons is different than the initial energy. The elastic measurement gives us information about the static magnetic properties of the material whereas, the inelastic measurement gives us information about the dynamic property of the material. 


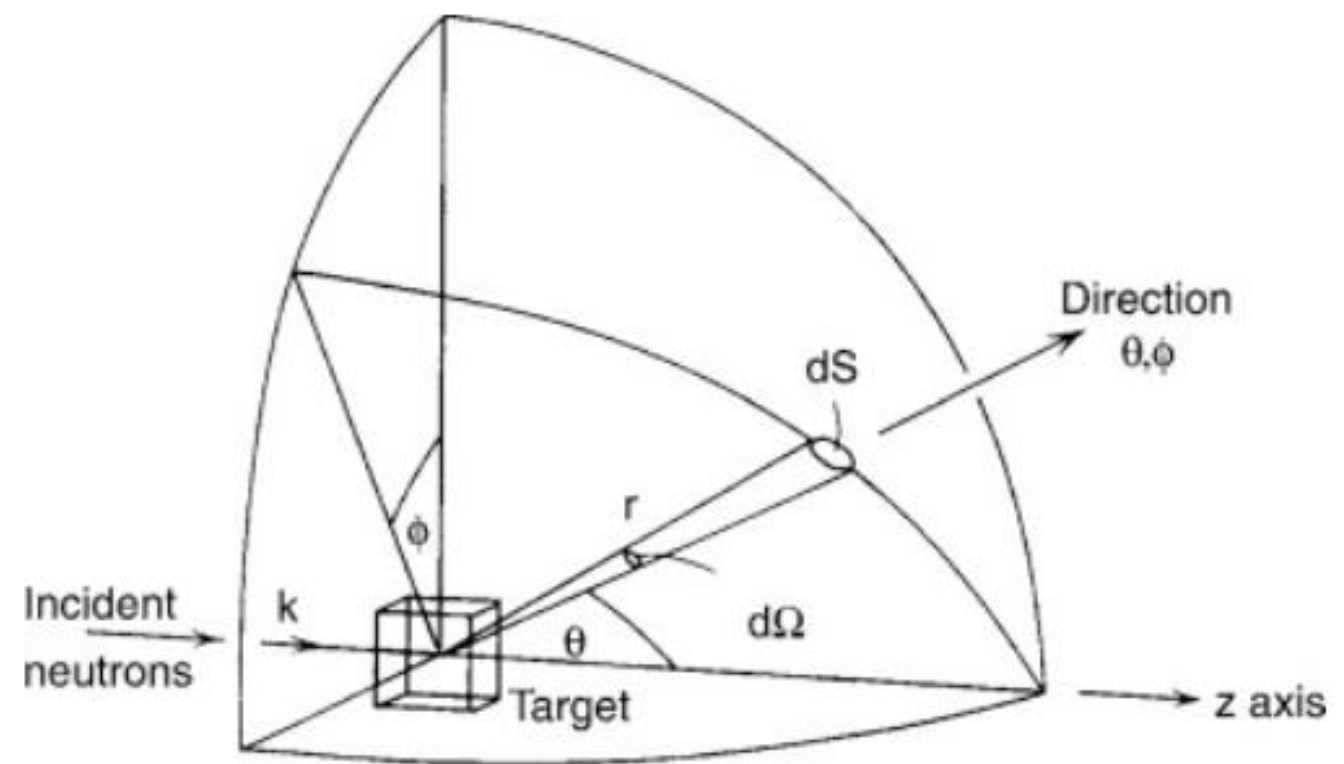

Figure 2.15: The figure shows the schematic of neutron scattering measurement. Here, $\Phi$ is the number of incident neutrons per $\mathrm{cm}^{2}$ per second, $\sigma$ is the total number of neutrons scattered per second $/ \Phi$. Reprinted with permission from $T$. Chatterji et. al [71]. Copyright 2019 by the Elsevier. 


\section{Elastic Neutron Scattering Measurements}

The diamagnetic response in NiSi that is frequency and temperature dependent is perplexing. Detailed neutron scattering measurement were performed in the system to gain insight into this anomalous behavior. The elastic measurement, which is used to detect any magnetic order in a material, show neither additional peaks beyond lattice peaks nor any temperature-dependent enhancement in the lattice peaks at low temperature (Fig. 2.16). We expect temperature dependent enhancement of structural peaks if we have ferromagnetic ordering in the sample as the magnetic ordering of the moments will contribute to the scattering. Similarly, an anti-ferromagnetic ordering will give peaks at different position than the structural peaks as the magnetic unit cell for the antiferromangetic configuration will be double that of the structural unit cell. Since none of the characteristics of magnetic ordering is present, we can rule out the possibility of any anti-ferromagnetic or ferromagnetic ordering in NiSi. 


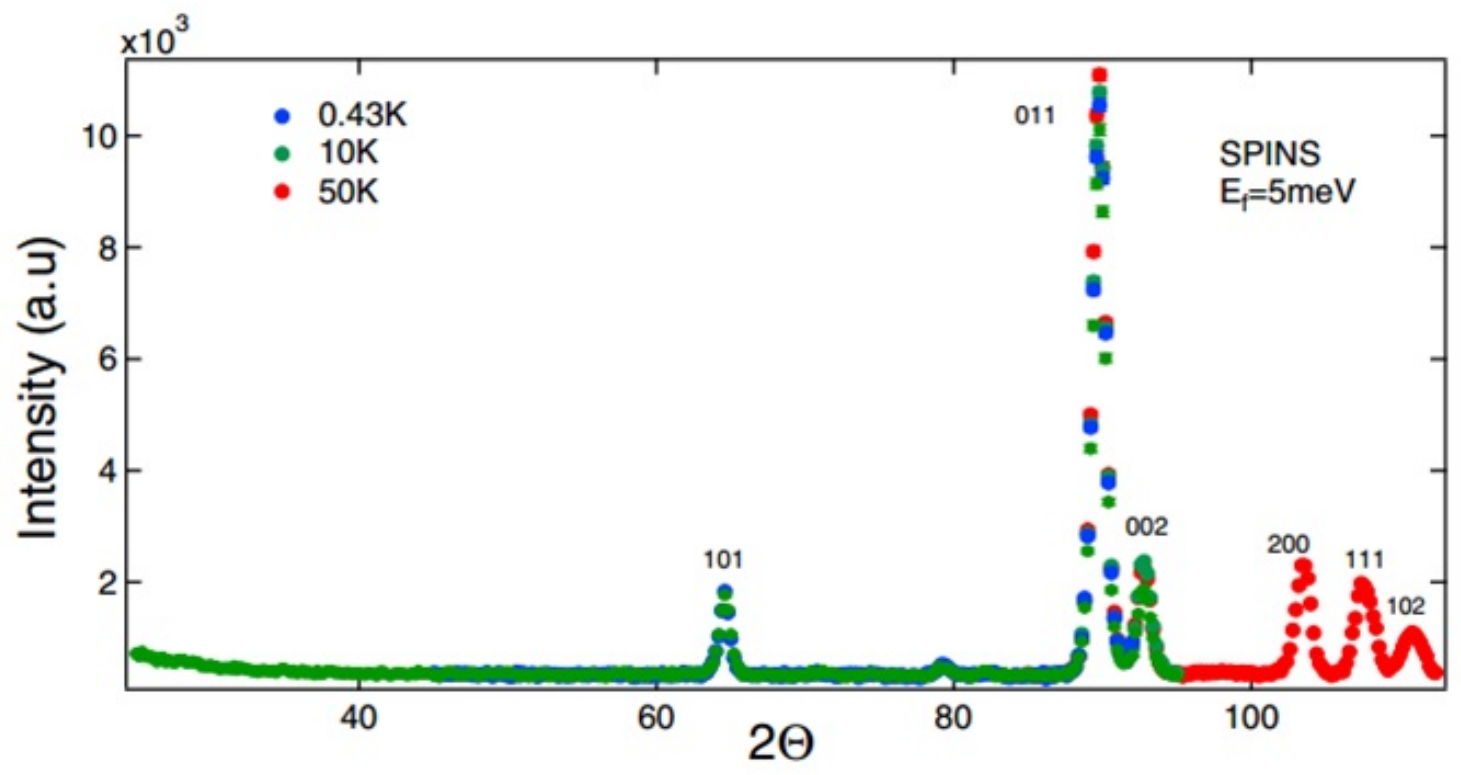

Figure 2.16: Elastic neutron scattering measurements on different compositions. High-resolution elastic neutron scattering measurements on powder NiSi were performed on the SPINS cold triple axis spectrometer at the NIST Center for Neutron Research. Observed sharp peaks in this figure are attributed to the nuclear structure factor. No additional peak, indicating antiferromagnetic order, is observed. Also, the difference between the elastic scans data at low temperature and high temperature is found to be statistically insignificant. Together, it rules out the presence of any magnetic order in NiSi. The error bar represents one standard deviation in the experimental data. 


\section{Inelastic Neutron Scattering Measurements}

A broad but well-defined peak centered at $E=1.75 \mathrm{meV}$ appears at low temperature in the background corrected inelastic neutron data. Inelastic neutron scattering measurements were performed on a $3.1 \mathrm{~g}$ pristine powder of NiSi on SPINS cold triple-axis spectrometer at the NIST Center for Neutron Research with a fixed final neutron en-

ergy of $5 \mathrm{meV}$, at which the spectrometers resolution (FWHM) was determined to be $\sim 0.28 \mathrm{meV} . \chi^{\prime \prime}(\mathrm{Q}, \mathrm{E})$ is derived from inelastic neutron data, using the following relation:

$$
S(Q, \omega)=\gamma_{0}^{2}\left(\frac{k_{i}}{k_{f}}\right) f(Q)^{2} \frac{1}{1-e^{\frac{-h \omega}{k_{B} T}}} \frac{\chi^{\prime \prime}(Q, \omega)}{\pi}
$$

where $\gamma_{0}^{2}=0.073 / \mu_{B}^{2}, k_{i}$ and $k_{f}$ represent initial and final neutron wave vectors, and $f(Q)$ is the form factor of magnetic ions (in this case $\mathrm{Ni}$ ion). Inelastic data were normalized by utilizing the incoherent scattering from a vanadium sample of known mass (also used to estimate the resolution of the instrument). Following the normalization procedure by $\mathrm{Xu}$ et al. [72], we write the normalized dynamic susceptibility as

$$
\chi "=\frac{\pi}{2} \mu_{B^{2}}\left(1-e^{\frac{-h \omega}{k_{B} T}}\right) S(Q, \omega)
$$

where $S(Q, \omega)$ can be written as

$$
S(Q, \omega)=\frac{13.77\left(\text { barns }^{-1}\right) \mu_{B}^{2} I(Q, E)}{\left|F(Q)^{2}\right| e^{-2 W} k_{i} R_{0}}
$$

The energy integrated incoherent scattering intensity

$$
\int I(Q, E) d E=\frac{N}{4 \pi} \Sigma_{j} \sigma_{j}^{i n c} e^{-2 W} k_{i} R_{0}
$$


was obtained by performing a $\Delta E=0$ scan at a wave vector $\mathrm{Q}$. Therefore, we have

$$
N k_{i} R_{0}=4 \pi \frac{\int I(Q, E) d E}{\Sigma_{j} \sigma_{j}^{i n c} e^{-2 W}}
$$

The variation of the Debye-Waller factor was neglected in the calculation. Vanadium sample with net mass of $0.0373 \mathrm{~mol}$ was used to estimate the total number of unit cells $(\mathrm{N})$ in $N_{\text {vanadium }} k_{f} R_{0}$. The incoherent inelastic scan on the vanadium sample was performed under the same instrument configuration as used in experiments on NiSi. In Fig 2.17, we show the normalized data for $T \sim 1.5 K$.

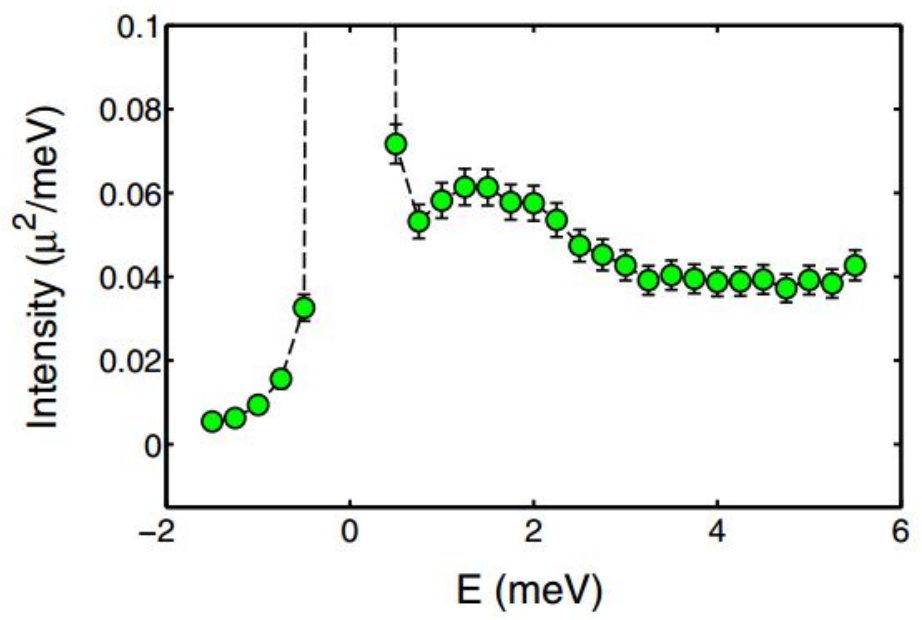

Figure 2.17: Full inelastic neutron scattering measurement data at $\mathrm{T}=1.5 \mathrm{~K}$. Full inelastic scan at $Q=1.03 \AA^{1}$ at $\mathrm{T}=1.5 \mathrm{~K}$ on NiSi is plotted here. The experimental data is background corrected, thermally balanced, and normalized with respect to the vanadium scattering of known mass. 


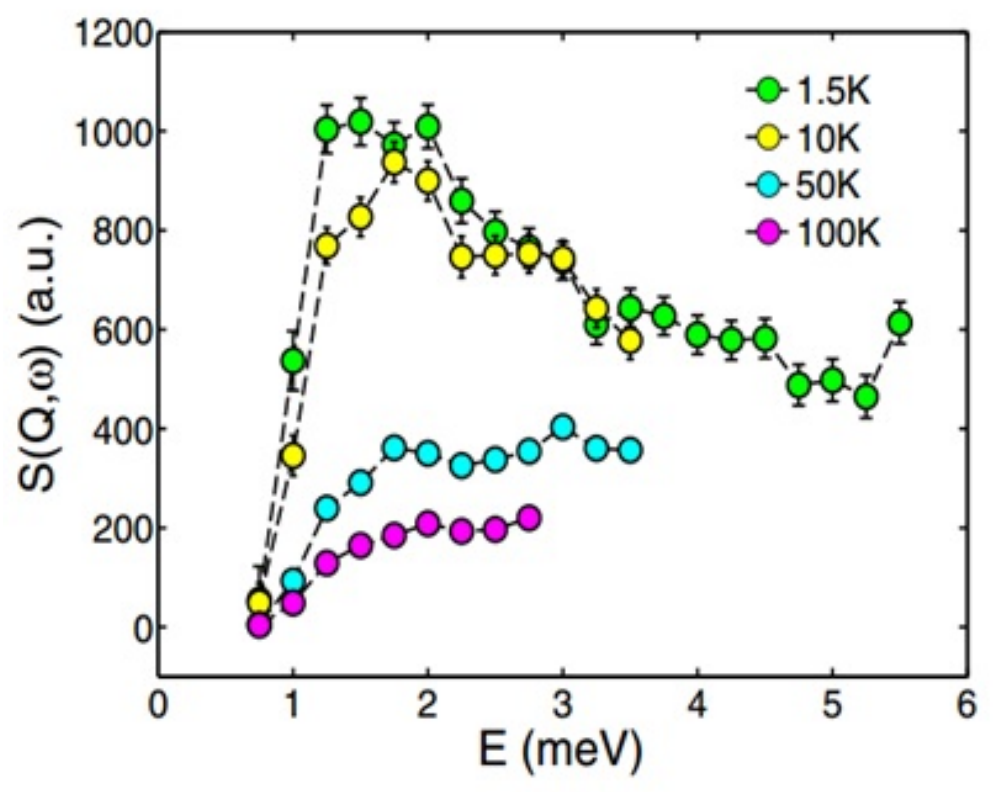

Figure 2.18: Inlelastic neutron scattering measurements on NiSi at different temperatures. Thermally balanced inelastic spectra at a fixed $Q=1.03 \AA$ at different temperatures are shown in this figure. For this purpose, the background corrected raw data is divided by the factor $\left[1-\exp \left(-E / k_{B} T\right)\right]^{-1}$. Inelastic measurements indicate the development of a gapped excitation centered at $E \simeq 1.75 \mathrm{meV}$. The error bar represents one standard deviation in the experimental data.

Figure 2.19 shows the dynamic susceptibility, $\chi "(Q, E)$ as a function of neutron energy transfer $E=E_{i}-E_{f}$ at different temperatures. The wave vector is $Q=$ 1.03 $\AA^{\circ}$. An inelastic peak in the dynamic susceptibility is observed that is centered at $E \simeq 1.75 \mathrm{meV}$, at the fixed wave vector. We observe a temperature dependence in the peak intensity. As the temperature increases, the peak intensity decreases. The temperature dependence of the dynamic susceptibility suggests magnetic nature of the dynamic properties.

The strong dynamic behavior in NiSi is present without any magnetic order. This kind of behavior is usually found in quantum magnets, for example a quantum spin liquid of a spin $-1 / 2$ system, or an unconventional superconductor of magnetic nature. $[29,73,38]$ In the latter case, an inelastic ${ }_{43}$ eak depicts a gapped magnetic resonance 
that exhibits a one-to-one correspondence with the superconducting order parameter. We have further analyzed $\chi^{\prime \prime}(Q, E)$ data to obtain more information about the system. An inelastic peak in $\chi^{\prime \prime}(Q, E)$ is fitted using a random phase approximation (RPA) model. The RPA has be previously used to successfully describe inelastic phenomena in Ni-based systems. It is also more accurate in describing the low temperature behavior. For the fitting, we make two assumptions: the only appreciable interaction is the nearest-neighbor interaction between $\mathrm{Ni}$ ions, $J_{1}$, and second, the interaction is isotropic in nature. Under these approximations, $\chi "(Q, E)$ is given by

$$
\chi^{\prime \prime}(Q, \omega)=\Sigma_{ \pm} \frac{\omega \chi_{0} \Gamma_{Q^{ \pm}}}{\Gamma_{Q^{ \pm}}^{2}+\omega^{2}}
$$

where

$$
\Gamma_{Q^{ \pm}}=\Gamma\left[1 \mp \chi_{0} J_{1}\right]
$$

Though it might not be the best possible model to describe the dynamic property of NiSi, RPA's simple relation clearly fits the inelastic peak $\chi^{\prime \prime}(Q, E)$ very well. The fitted value of $J_{1}=0.93 \mathrm{meV} / \mu_{B}^{2}$ at $T=1.5 \mathrm{~K}$ indicates a modest exchange interaction between $\mathrm{Ni}$ ions. The values of $\Gamma$ and $\chi_{0}$ are plotted as a function of temperature in $(2.20)$, respectively. The dynamic correlation of $\mathrm{Ni}-\mathrm{Ni}$ ions are given by $\chi_{0}$. The correleation decreases sginificantly as the temperature approaches $\sim 100 \mathrm{~K}$. While at the same time, $\Gamma$, representating the full width at half maximum of dynamic correlation or the inverse of relaxation time $\tau$, increases as a function of temperature. This means that the magnetic fluctuation has a shorter relaxation time at higher temperature. The magnetic nature of the dynamic susceptibility is further verified from the $\mathrm{Q}$ dependent nature of the peak. At $Q=1.45 A^{-1}$, a significant reduction in peak intensity is observed. 


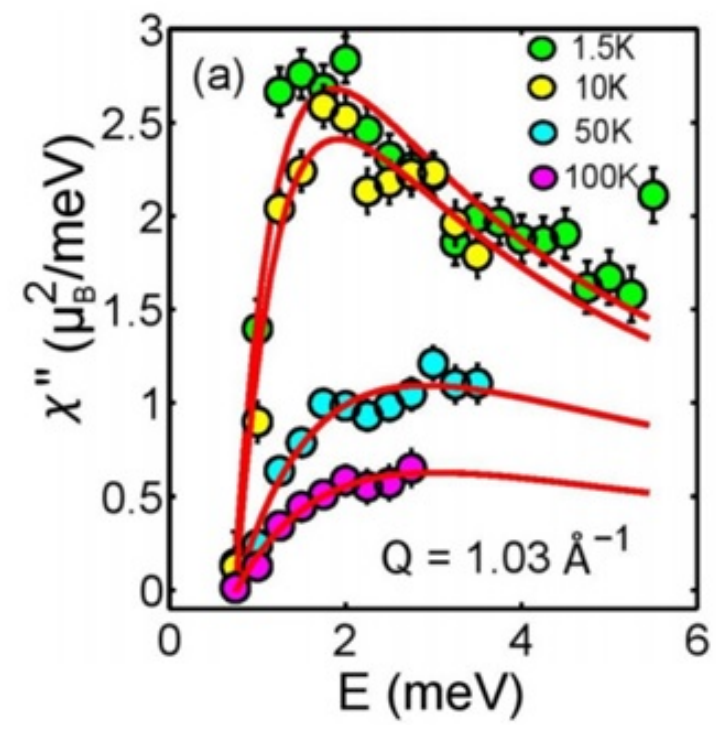

Figure 2.19: $\chi^{\prime \prime}(Q, E)$ is deduced from inelastic neutron scattering data as a function of energy transfer, $E=E i-E f$. Inelastic data were background corrected and normalized with respect to the incoherent scattering from a vanadium sample of known mass. The error bar represents one standard deviation in the experimental data. $\chi^{\prime \prime}(Q, E) v s E$ at a few different temperatures at $Q=1.03 \AA^{-1}$ are shown in this plot. Experimental data are well described by RPA analysis. The peak in $\chi "(Q, E)$ is centered at $E=1.75 \mathrm{meV}$ and is temperature dependent. 

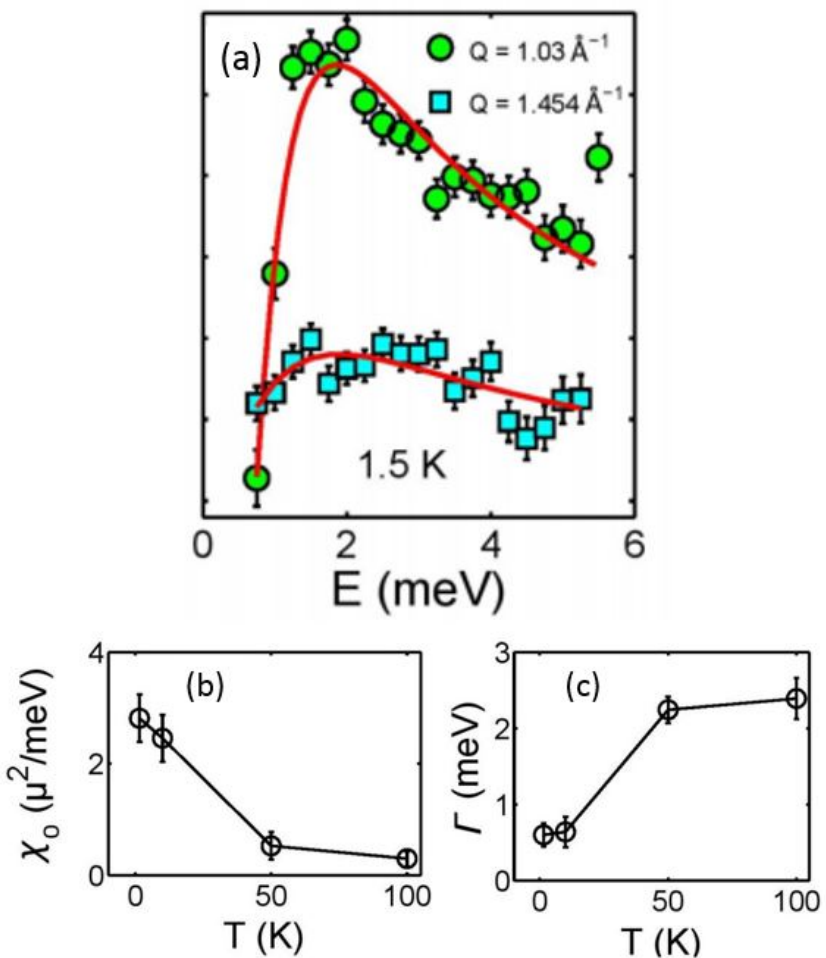

Figure 2.20: (a) $\chi$ " $(Q, E)$ vs $E$ at two different wave vectors of $Q=1.03$ and $1.45 \AA^{-1}$ at $T=1.5 \mathrm{~K}$ are shown in this figure. The peak intensity follows the Ni form factor. Along with the temperature dependence in Fig.2.19, it indicates magnetic nature of the excitation. The peak in $\chi$ " is centered at the same energy at both wave vectors. The inset shows the isotropic nature of the nearest-neighbor exchange interaction. (c) and (d) Obtained values of fitting parameters $\chi_{0}$ and $\Gamma$ (full width at half maximum) are plotted as a function of temperature. $\chi_{0}$ decreases as the measurement temperature increases and becomes very small at $T \simeq 100 K$. 


\subsection{Results and Discussion}

The peculier anomalous observation made in NiSi has not been previously reported on any other available literature. Generally, a metal tend to behave like a paramagnet or a diamagnet in an applied field, depending on the parallel or antiparallel alignment of electrons moment to the applied field, respectively $[74,75,76]$. The quantum-mechanical treatment of the metals bulk properties forbids any frequency or temperature dependence of the diamagnetic response in an ac magnetic field [74, 77]. It can be argued that the underlying Fermi surface in NiSi evolves as a function of temperature in applied field. This can cause a temperature-dependent diamagnetism. However, such reconstruction requires a large magnetic field [78]. We note that both anomalies are produced at very small magnetic fields (a few Oe to a few hundred Oe), not sufficient to induce the evolution of the Fermi surface as observed in the de Haasvan Alphen effect measurements. Moreover, the ac frequency dependence of the bulk susceptibility and the irreversibility in the ZFC and FC responses in NiSi sets it apart from a high magnetic field induced behavior in a metallic magnet. Another effect that can cause diamagnetism in a metal is the skin effect. However, the analytical calculation as well as the measurement with non magnetic epoxy $\mathrm{B}_{4} \mathrm{C}_{3}$ clearly rules out the role of skin effect in the diamagnetic properties of NiSi.

The minority superconducting phase, coexisting with the majority metallic phase in $\mathrm{NiSi}$, is apparent from detailed experimental observations. For instance, electrical measurements on NiSi depict a small downward cusp at $T \simeq 75 \mathrm{~K}$ in otherwise metallic characteristic as a function of temperature. This temperature is similar to the onset temperature of simultaneous maximization of the diamagnetic static susceptibility and the peak-type feature in the dynamic susceptibility in the ac susceptibility measurement, suggesting the presence of a superconducting phase in the system. Also, NiSi exhibits significant diamagnetic response in dc susceptibility measurements below $T \simeq 55 K$. It is also found that the irreversibility between $\mathrm{ZFC}$ and $\mathrm{FC}$ curves 
is much stronger at low field, compared to high field. However, the net susceptibility at low temperature $\left(4 \pi \chi \simeq 10^{-4}\right)$ is much less than that of a perfect superconductor $(4 \pi \chi=-1)$, indicating the minority character of the superconducting phase. We note that the diamagnetic irreversibility between ZFC and FC curves follows a peak-type feature at $T \simeq 55 \mathrm{~K}$. While its origin is not yet understood, it may be arising due to the competing effects between the weak paramagnetic nature of the bulk and the minority-superconducting phase that does not percolate to bulk. These arguments infer that NiSi apparently represents a new intermediate regime between a metal and a superconductor, which shares its traits with both states. 


\section{Chapter 3}

\section{Quantum Magnetic Properties in Co doped $\mathrm{CaRuO}_{3}$}

Geometrically frustrated lattice in spin- $1 / 2$ magnet tend to give rise to Quantum magnetic properties like quantum spin liquid or solid. We have found that controlled chemical disorder created by substitution of $\mathrm{Ru}$ ions by Co-ions, in perovskite $\mathrm{CaRuO}_{3}$ creates a random configuration of artificial spin- $1 / 2$ that form dimer pairs between the nearest and further away ions. The dimers of artificial spin-1/2, due to localization of Co impurities, exhibit singlet-to-triplet excitation at low temperature without any ordered spin correlation. The localized gapped excitation evolves into a gapless quasi-continuum as dimer pairs break and create freely fluctuating spins at high temperature.

\subsection{Sample Preparation}

The samples of $\mathrm{Ca}\left(\mathrm{Co}_{x} \mathrm{Ru}_{1-x}\right) \mathrm{O}_{3}$ were synthesized by conventional solid state reaction method using ultrapure ingredients of $\mathrm{CoO}, \mathrm{RuO}_{2}$, and $\mathrm{CaCO}_{3}$. Starting materials were mixed in stoichiometric composition, pelletized, and sintered at $950^{\circ}$ for 3 days 
in oxygen-rich environment. The sample was furnace cooled. After that the samples were grinded, pelletized, and sintered at $1000^{\circ}$ for another 3 days. Samples were intentionally synthesized at slightly lower temperature and for longer duration to preserve the oxygen stoichiometry.

\subsection{Sample Characterization}

Samples were characterized using Siemens D500 powder X-ray diffractometer (XRD), confirming the single phase of material. The X-ray diffraction data were analyzed using the FullProf suite for the Reitveld refinement, confirming the high quality single phase of materials. Every single peak of the XRD pattern is identified with the orthorhombic structure of $\mathrm{Ca}\left(\mathrm{Co}_{x} \mathrm{Ru}_{1-x}\right) \mathrm{O}_{3}$. 


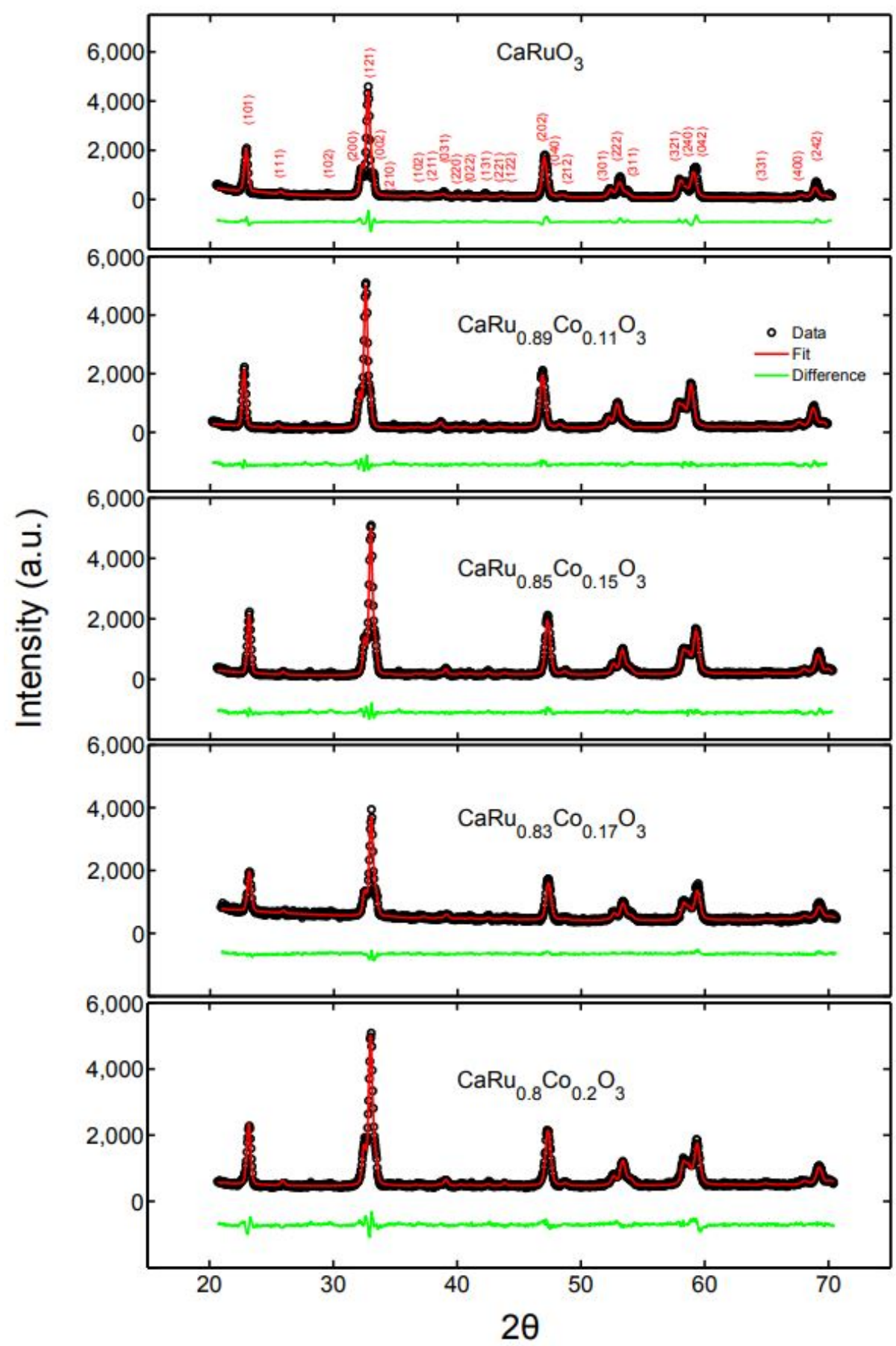

Figure 3.1: XRD profiles of $\mathrm{Ca}\left(\mathrm{Co}_{x} \mathrm{Ru}_{1-x}\right) \mathrm{O}_{3}$. Powder Xray diffraction spectra of $\mathrm{Ca}\left(\mathrm{Co}_{x} \mathrm{Ru}_{1-x}\right) \mathrm{O}_{3}$ powder, for various substitution coefficient $\mathrm{x}$, used in this study. The powder diffraction data is refined using FullProf suite for the Rietveld analysis. The high purity of the sample is evident from the XRD data, where every single peak is identified with the orthorhombic structure of $\mathrm{CaRuO}_{3}$. 


\subsection{Electrical Measurements}

We performed electrical measurements for different sample with various Co substitutions using four probe measurement technique. The samples showed metal to insulator transition as we increased the Co substitution. The metal-insulator transition continued until $\mathrm{x}=0.15$. The electrical resistance was found to obey the Arrhenius-type activation in compounds with $\mathrm{x}>0.15$. Thus, confirming the insulating characteristic of the system where the underlying physics is appropriately described by the Heisenberg formulation. [79, 19]

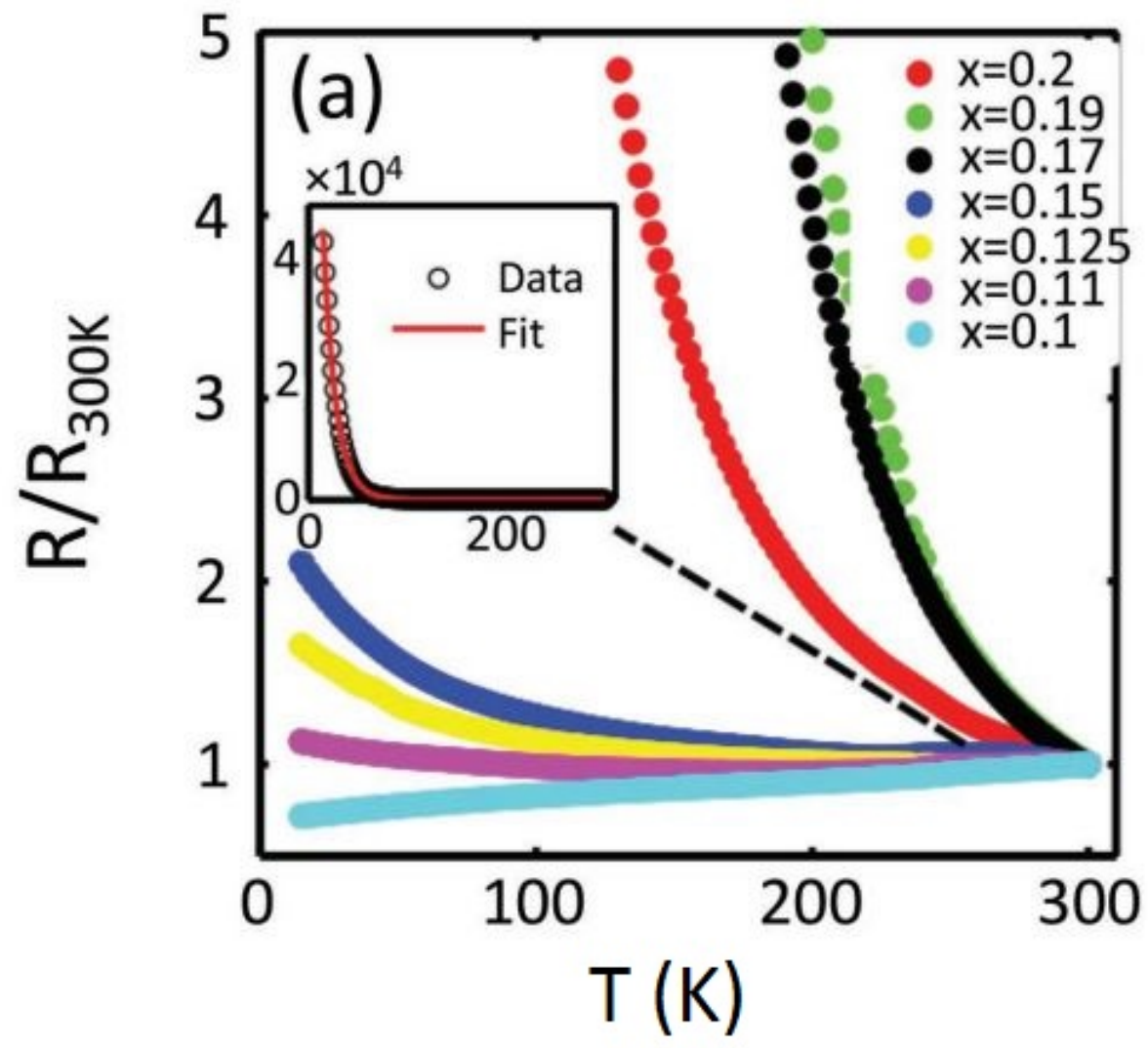

Figure 3.2: Plot of electrical resistivity versus temperature for various $\mathrm{x}$. As $\mathrm{x}$ increases, a transition from metal to insulator phase is clearly observed. At x $>0.15$, the system enters to the activated insulating regime. Inset shows the Arrhenius formulation-type activation in $\mathrm{x}=0.19$. 


\subsection{Magnetization Measurements}

The magnetization measurements were performed using QDPPMS with various Co substituted samples. The measurement were performed at frequency range of 500$10^{4} \mathrm{~Hz}$. The measurements reveal strong frequency dependent dynamic susceptibility in Co substituted $\mathrm{CaRuO}_{3}$. A small cusp is observed to develop at $\mathrm{T} \simeq 70 \mathrm{~K}$ in $\chi^{\prime}$ as $\mathrm{x}$ increases. However, the overall increase in $\chi^{\prime}$ at low temperature is marginal compared to the value at $\mathrm{T}=300 \mathrm{~K}$. Thus, the system exhibits weak paramagnetic character at low temperature. Unlike $\chi^{\prime}, \chi^{\prime \prime}$ exhibits considerable reversal in the temperature dependence across the critical substitution $\mathrm{x}=0.15$. At substitution of $\mathrm{x}<0.15$ we get a strong frequency dependence for high frequency at low temperature. However, as we increase the doping concentration to be $\mathrm{x} \geq 0.17$, we get stronger frequency dependence at high temperature. For example, in $\mathrm{x}=0.17$, $\chi$ " is much stronger at room temperature compared to the almost negligible magnitude at low temperature. The completely surprising observation in $\chi$ " at high $\mathrm{x}$ can be ascribed to two possibilities: first, the fluctuation at high temperature is Q-independent, which coalesce to finite wave vectors in the momentumenergy (QE) space as temperature is reduced. At high temperature, those localized excitation melt in the broader realm of the QE space. Such a scenario will give rise to the continuum spectrum in QE space at high temperature. Alternatively, the system fluctuates at much faster rate at low temperature and, therefore, cannot be detected by the ac susceptibility probe. 

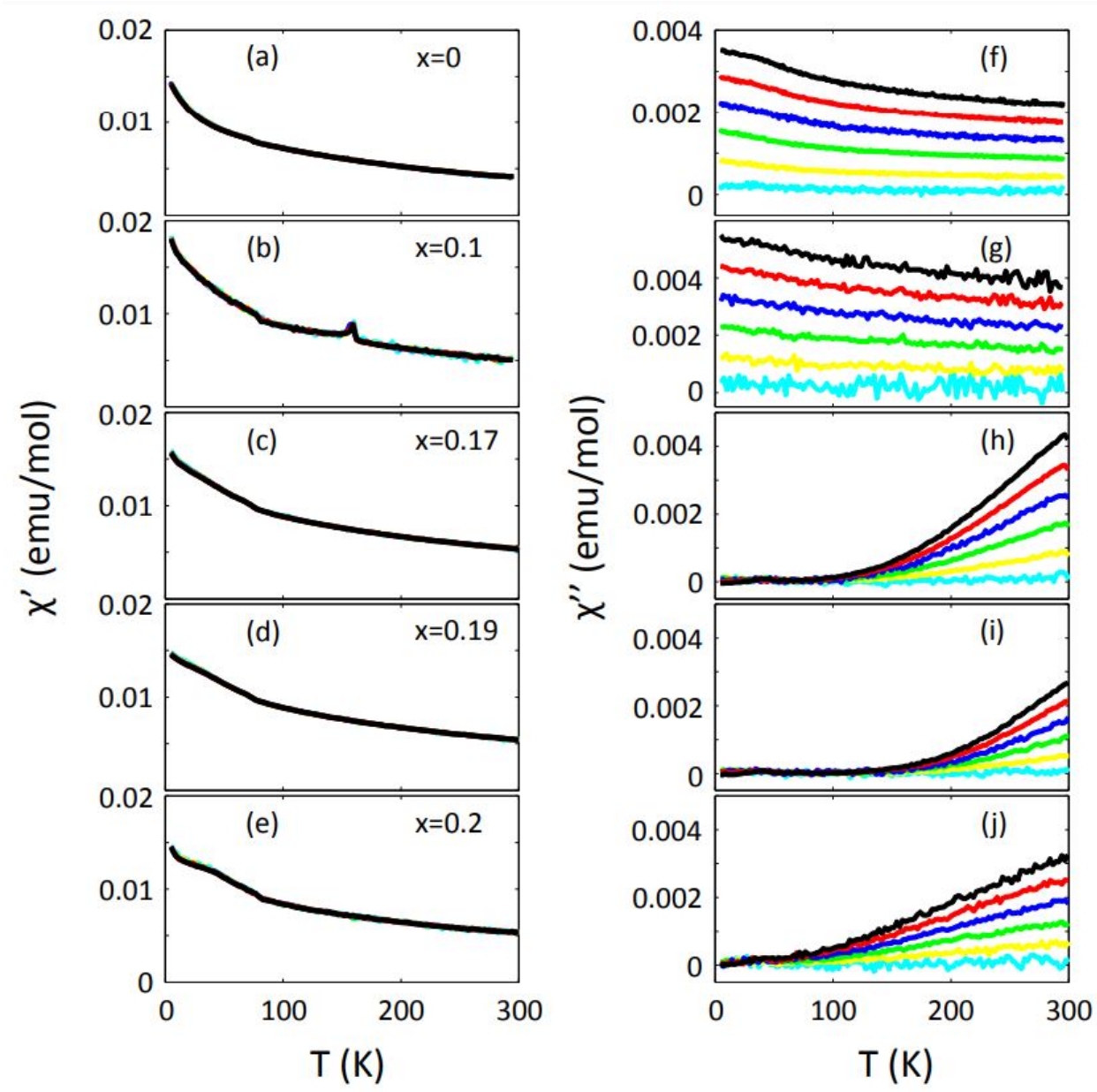

Figure 3.3: The static and dynamic susceptibilities as a function of temperature at different frequencies between $500-10^{4} \mathrm{~Hz}$, as obtained from the ac susceptibility measurements, for many substitution coefficients. While the static susceptibilities are ac frequency independent, the dynamic susceptibilities exhibit strong frequency dependences. Most notably, the $\chi$ " exhibits a complete reversal in the fluctuation spectrum as a function of frequency across the critical doping $\mathrm{x}=0.15 . \quad \chi$ " is much stronger at higher temperatures in $\mathrm{x}>0.15$. 


\subsection{Neutron Scattering Measurements}

\subsubsection{Elastic Neutron Scattering Measurements}

$2 \Theta$-scans were carried out on a thermal triple-axis spectrometer TRIAX at the Missouri University and on a cold triple axis spectrometer, SPINS, at the NIST Center for Neutron Research. Measurements on the thermal spectrometer was performed with a fixed final energy of $14.7 \mathrm{meV}$ and the collimation setting of $60-$ $P G$ filter monochromator - PG (pyrolitic graphite)filter - 80 - sample - $40-$ $P G$ filter - PG analyzer 80 - detector. Measurements on SPINS were performed at the fixed final energy of $\mathrm{Ef}=5 \mathrm{meV}$ with the collimators sequence of $P G$ (mono) Be Filter - Sample - BeO filter - 80 - flat analyzer - Detector. No evidence of magnetic order is found in compounds with $\mathrm{x}>0.15$. 

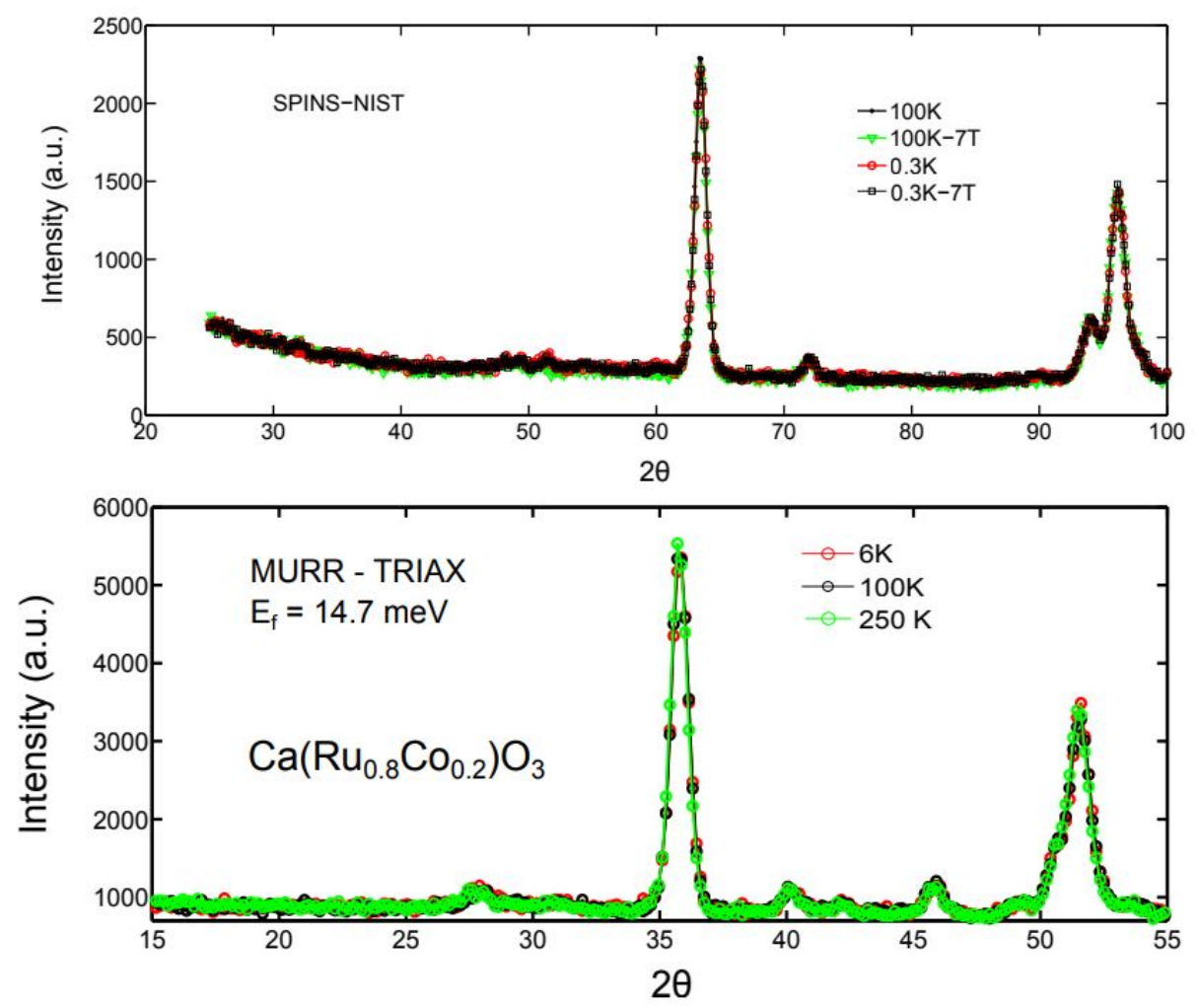

Figure 3.4: Top panel- Elastic measurements performed on the substitution coefficient $\mathrm{x}=0.17$ on a cold spectrometer SPINS using high resolution collimation and filters, with fixed final energy of $\mathrm{E}_{f}=5 \mathrm{meV}$, to a low temperature of $\mathrm{T}=0.3 \mathrm{~K}$ and in applied field of $\mathrm{H}=7 \mathrm{~T}$. Elastic neutron scattering pattern at various temperatures and fields are presented in this figure. Clearly, no extra peak, beyond the lattice peaks, is detected. Also, no enhancement in the lattice peaks or a diffuse background associated to the elastic peak are observed at low temperature. Together, they rule out any magnetic order in the system. Bottom panel- Elastic measurements performed on a thermal triple axis spectrometer TRIAX (at MURR) on the substitution coefficient $\mathrm{x}=0.2$ further confirms the absence of magnetic order in $\mathrm{Ca}\left(\mathrm{Co}_{x} \mathrm{Ru}_{1-x}\right) \mathrm{O}_{3}$. 


\subsubsection{Inelastic Neutron Scattering Measurements}

The temperature and magnetic field dependences of the dynamic properties were investigated in various compositions of $\mathrm{Ca}\left(\mathrm{Co}_{x} \mathrm{Ru}_{1-x}\right) \mathrm{O}_{3}$ using inelastic neutron scattering measurements on the SPINS cold triple-axis spectrometer and MACS spectrometer at the NIST Center for Neutron Research. The spectrometers configuration for inelastic measurements on SPINS was PG(mono $)-80$ - Sample - BeOfilter radialcollimator - 10 blades focused analyzer - Detector. The spectrometers resolution was determined using an empty vanadium can scan. At $\mathrm{E}_{f}=5 \mathrm{meV}$, the spectrometers resolution was determined to be $\simeq 0.28 \mathrm{meV}$. The spectrometers configuration on MACS was $P G($ mono $)-80$ - Sample-Be filter-radial collimatorfocused analyzer - 20detectors. Measurements were performed for the fixed final energy of $\mathrm{E}_{f}=5 \mathrm{meV}$, at which the spectrometers resolution was determined to be $\simeq 0.32 \mathrm{meV}$. In Fig.3.7, we plot the raw data at two characteristic measurement temperatures of $\mathrm{T}=30 \mathrm{~K}$ and $80 \mathrm{~K}$ at two magnetic fields of $\mathrm{H}=0 \mathrm{~T}$ and $\mathrm{H}=$ 9 T, the background data and the background corrected data. Fig.3.5 depicts measurements on SPINS in both zero and applied field of $\mathrm{H}=10 \mathrm{~T}$ at two characteristic temperatures of $\mathrm{T}=5 \mathrm{~K}$ and $100 \mathrm{~K}$. 


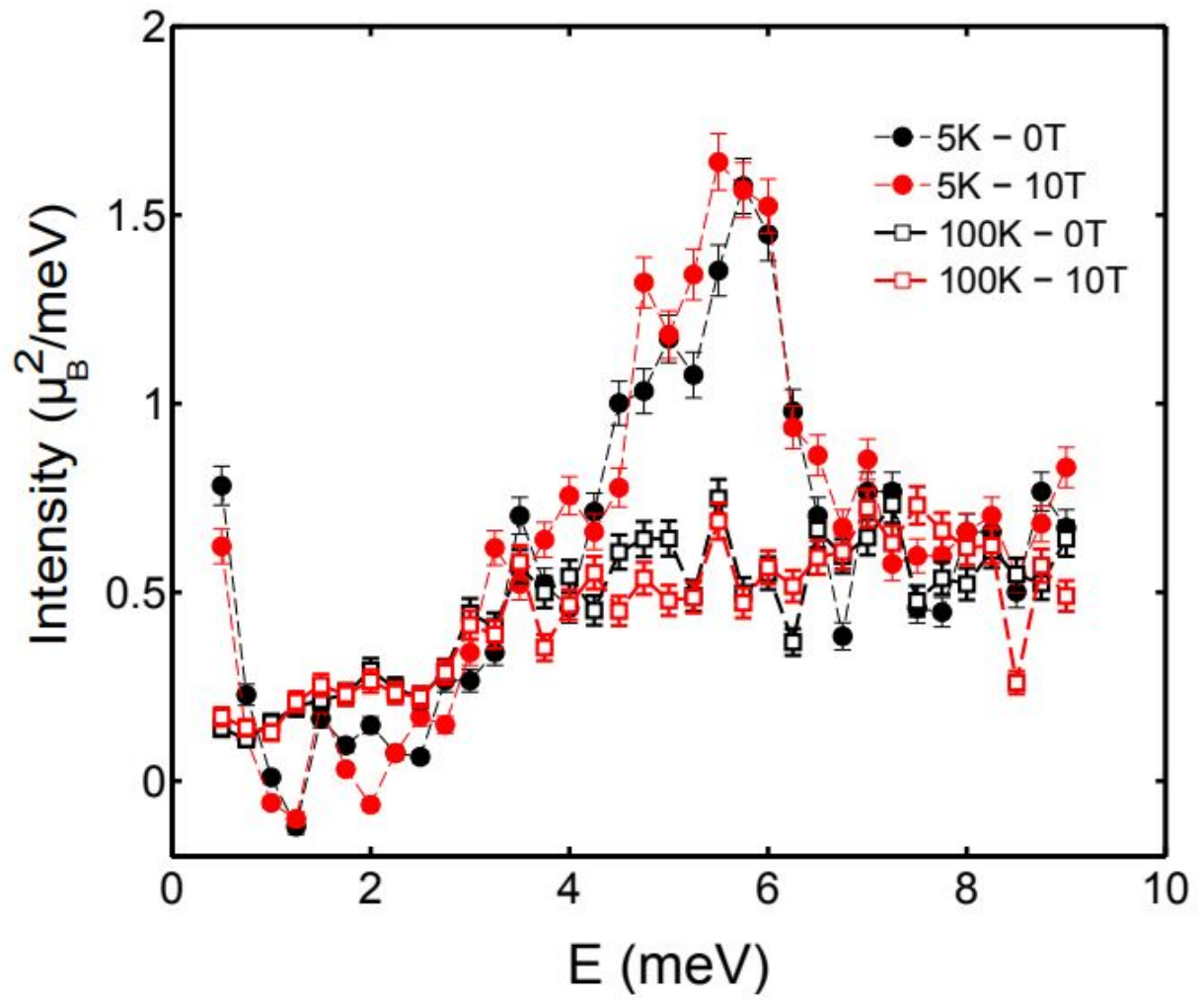

Figure 3.5: Background corrected inelastic data in both zero and applied field of $\mathrm{H}$ $=10 \mathrm{~T}$ at $\mathrm{T}=5 \mathrm{~K}$ and $100 \mathrm{~K}$. Clearly, no field dependence is observed at any temperature. Measurements were performed on the SPINS cold triple axis spectrometer. 


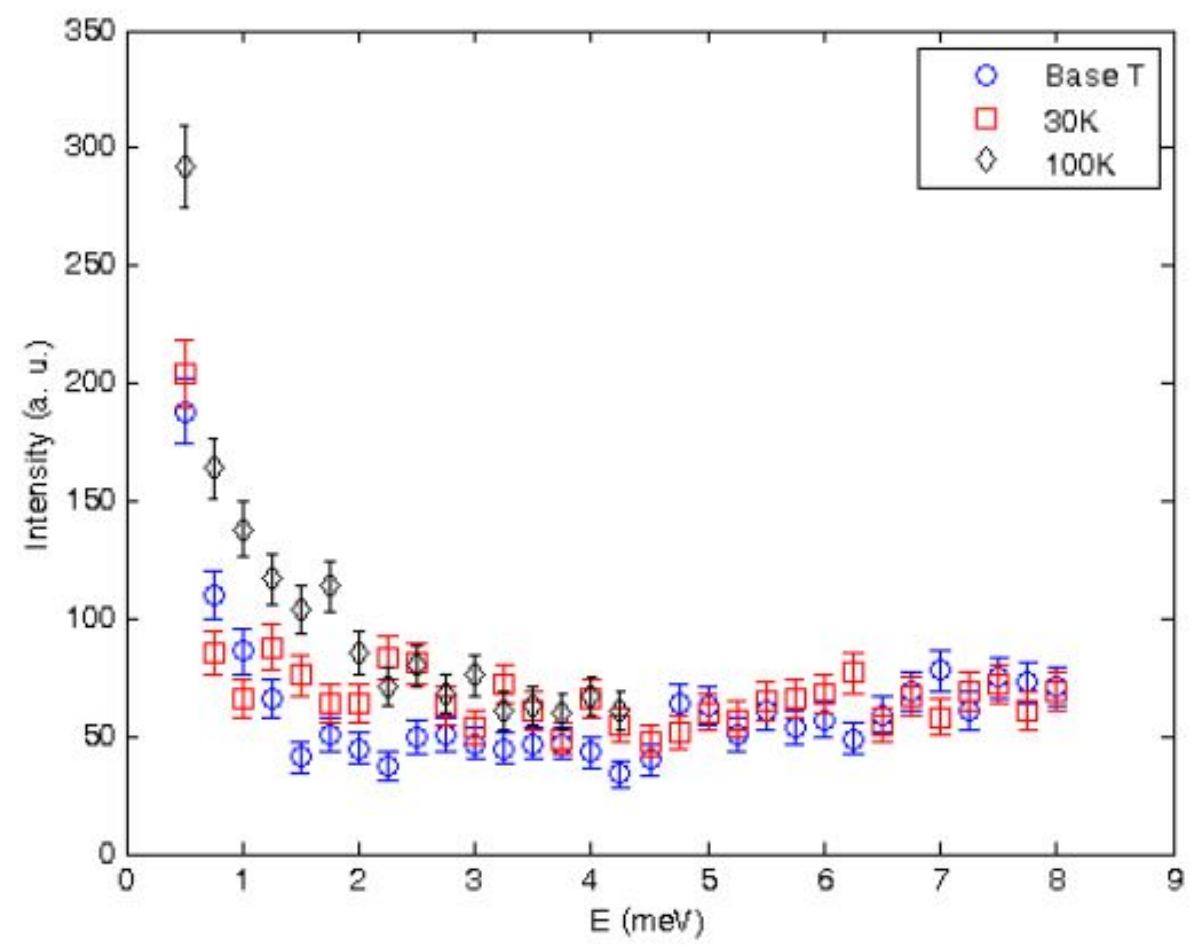

Figure 3.6: Inelastic measurement data on $\mathrm{CaRuO}_{3}$. Measurements were performed at $\mathrm{T}=1.5 \mathrm{~K}$ (base temperature), $30 \mathrm{~K}$ and $100 \mathrm{~K}$. Unlike $\mathrm{Ca}\left(\mathrm{Co}_{x} \mathrm{Ru}_{1-x}\right) \mathrm{O}_{3}$, no excitation at finite energies are observed at any temperature in the stoichiometric compound $(\mathrm{x}=0)$. 


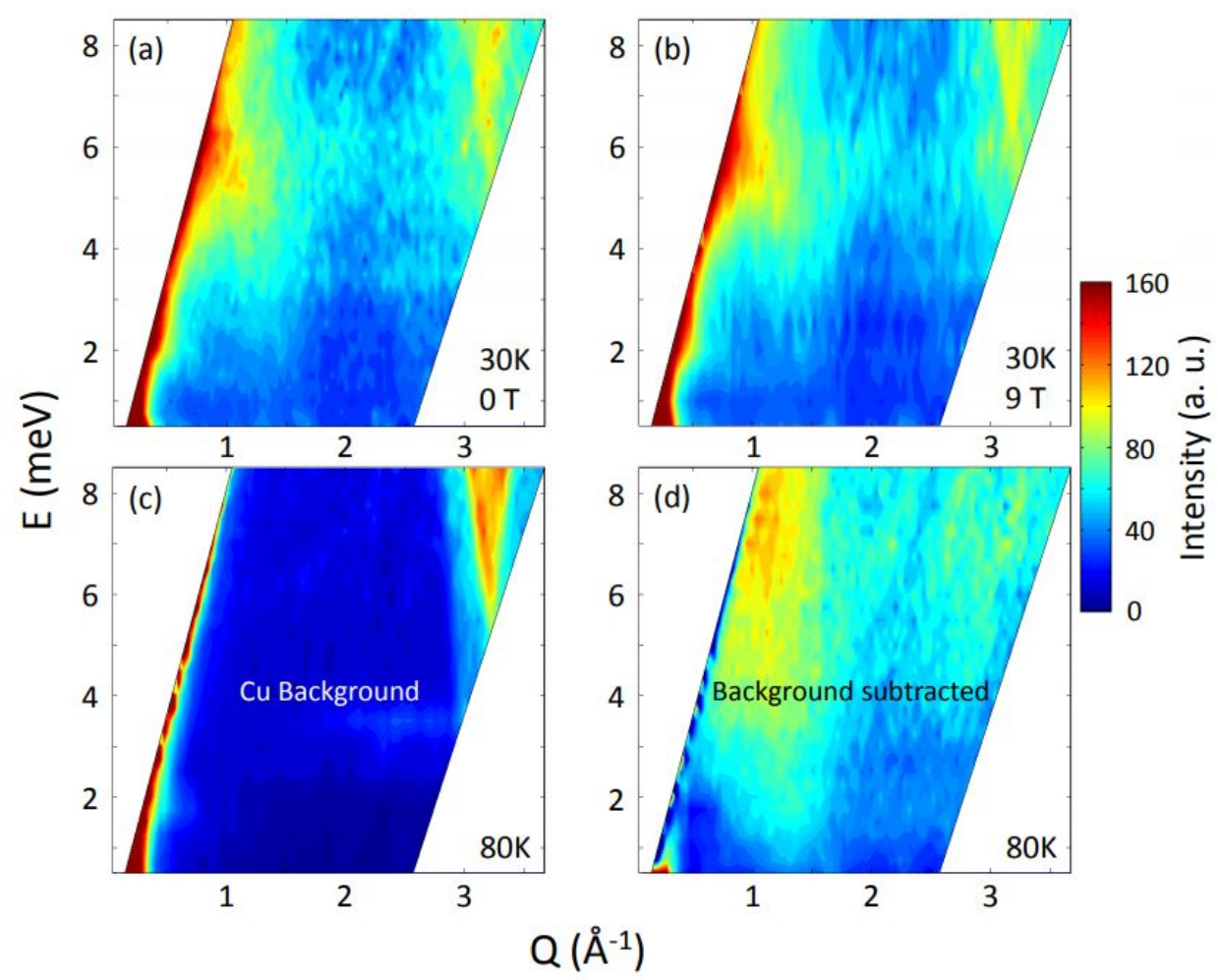

Figure 3.7: Color map of spin fluctuation in energy momentum space, as obtained from the MACS measurements. (a) and (b) represents raw data at $\mathrm{T}=30 \mathrm{~K}$ at $\mathrm{H}$ $=0 \mathrm{~T}$ and $9 \mathrm{~T}$, respectively. (c) $\mathrm{Cu}$ background data at $\mathrm{T}=80 \mathrm{~K}$. Sharp scattering at high Q is due to copper can. (d) Background corrected and thermally normalized data at $\mathrm{T}=80 \mathrm{~K}$.

Two types of trends are immediately observed in the Q-E maps as shown in Fig. 3.8: first, at low temperature of $\mathrm{T}=80 \mathrm{mK}$, a well-defined gapped excitation at $\mathrm{E}=5.9 \mathrm{meV}$ develops at $\mathrm{Q}=1 \AA^{-1}$. The dynamic structure factor is much broader than the instrument resolution in both momentum and energy space (Figure $3.8 \mathrm{a}-\mathrm{c})$. Also observable is a much weaker excitation at $\mathrm{Q}=2 \AA^{-1}$ at the same energy, which follows Co form factor. Given the fact that no magnetic order was detected in any of these materials, the observation of localized excitation is completely surprising. The excitation is non-dispersive in nature. No such localized excitation was observed in $\mathrm{CaRuO}_{3}$ or $\mathrm{Ca}\left(\mathrm{Co}_{x} \mathrm{Ru}_{1-x}\right) \mathrm{O}_{3}$ with low substitution coefficient (see Figure 3.6). [11] It further suggests that the gapped excitation arises due to the 
$\mathrm{Co}^{4+}-\mathrm{Co}^{4+}$ interaction. As temperature increases, the excitation gradually weakens before becoming indistinguishable from the remnant background.
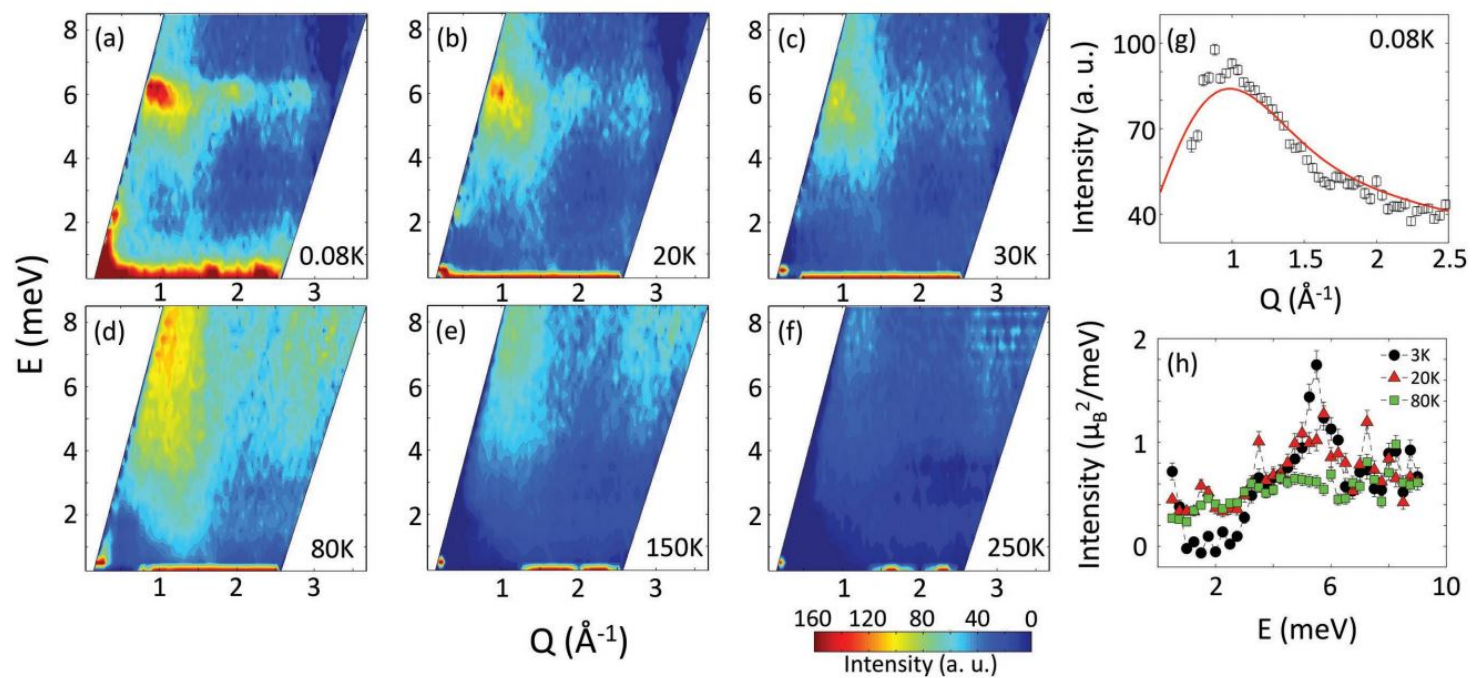

Figure 3.8: Localized excitation from dimerdimer interaction to quasi-continuum spectrum. a-c) Energymomentum (QE) map, depicting localized excitation in $\mathrm{Ca}\left(\mathrm{Co}_{0.17} \mathrm{Ru}_{0.83}\right) \mathrm{O}_{3}$, at different temperatures, ranging from $\mathrm{T}=80 \mathrm{mK}$ to $30 \mathrm{~K}$, are plotted. Experimental data are background corrected and thermally balanced by multiplying with the Bose factor, $\left(1-\exp ^{(-E / k B T)}\right)$. A well-defined excitation is found to be centered at $\mathrm{E}=5.9 \mathrm{meV}$ and $\mathrm{Q}=1$ and $2 \AA^{-1}$, albeit weakly. It follows the form factor of Co. The excitation becomes weaker as temperature increases. $d-f$ ) As temperature increases further, the excitation reappears. However, it is much more broader in both momentum and energy, reminiscent of a quasi-continuum behavior. At $\mathrm{T}=80 \mathrm{~K}$, the quasi-continuum fills the majority of the $\mathrm{Q}-\mathrm{E}$ space, which persists to an unusually high temperature of $\mathrm{T}=250 \mathrm{~K}$. g) At $\mathrm{T}=80 \mathrm{mK}$, the averaged intensity versus $\mathrm{Q}$ is well described by a singlet-to-triplet transition due to the dimer-dimer interaction between nearest and further away spins. (h) Measurements on SPINS at $\mathrm{Q}=1.15 \AA^{-1}$ provide more quantitative perspective. 
The collective gapped excitation at low temperature (Figure3.8 a-c), centered at $\mathrm{E}=5.9 \mathrm{meV}$, is comparable to the mean field exchange constant of $\mathrm{Co}-\mathrm{Co}$ interaction, $\mathrm{J}_{C o-C o} \simeq 6 \mathrm{meV}$. The broad width of the inelastic peak suggests a continuum of excitation, involving exchange constants beyond nearest neighbors. To understand the origin of this collective excitation, we plot the averaged intensity of the inelastic spectra between $\mathrm{E}=4.5$ and $7.5 \mathrm{meV}$ as a function of the wave-vector $\mathrm{Q}$ at $\mathrm{T}=80 \mathrm{mK}$. As shown in Figure $3.8 \mathrm{~g}$, the experimental results are well described by the singletto-triplet excitation of spin dimer, involving nearest and the next nearest neighbor interactions $[30,31]$ :

$$
I(Q)=|F(Q)|^{2} \Sigma m_{i}^{2}\left[1-\sin \left(Q d_{i}\right) /\left(Q d_{i}\right)\right]
$$

where $m_{i}^{2}$ is the squared moment per formula unit and $d_{i}$ is distance between nearest or next nearest neighbor. Simple inclusion of the nearest neighbor interaction does not fit the data well.[31, 80] The estimated values of $m_{i}^{2}$ and $d_{i}$ at $\mathrm{T}=80 \mathrm{mK}$ are: $m_{1}^{2}=0.367 \mu B^{2}, d_{1}=3.85 \stackrel{\circ}{A}, \mathrm{~m}_{2}^{2}=0.15 \mu B^{2}$ and $\mathrm{d}_{2}=5.4 \stackrel{\circ}{A}$. The near neighbor distances, $\mathrm{d}_{1}$ and $\mathrm{d}_{2}$, are remarkably close to the lattice values. The estimated dimer moments are also consistent with similar observations in the chemically ordered spin1/2 systems, such as the Kagome structure.[29] As temperature increases, the dynamic structure factor gradually decreases and disappears above $\mathrm{T}>30 \mathrm{~K}$.

Second, the excitation spectra re-emerges near the same wavevectors but centered at nominally higher energy $\mathrm{E} \simeq 7.8 \mathrm{meV}$, as temperature is further increased to $\mathrm{T}$ $=80 \mathrm{~K}$ (Figure $3.8 \mathrm{~d}-\mathrm{f}$ ). Most importantly, the high temperature excitations are gapless and occupy a significant portion of the QE space. The broad continuous tail in both energy and the momentum space is reminiscent of the quasi-continuum spectrum, observed in geometrically frustrated lattice of spin-1/2. [28, 81] The quasicontinuum excitation at high temperature complements the ac dynamic susceptibility 
measurements in $\mathrm{x}>0.15$ (Figure 3.3). As we cross into the Heisenberg regime, i.e., $\mathrm{x}>0.15$, the dynamic susceptibility pattern changes significantly. The fluctuation is now confined to higher temperature only (Figure 3.3). It confirms the gapped nature of the excitation at low temperature and the continuum spectrum at higher temperature. Although, the spectral weight is distributed in the large region of $\mathrm{QE}$ space, the remnants of localized excitation at $\mathrm{Q}=1$ and $2 \stackrel{\circ}{A}^{-1}$ can still be seen, albeit much weaker, at higher temperature. It further suggests that the quasi-continuum is arising due to the breaking of the dimer pairs. These excitation persist to very high temperature, $\mathrm{T} \simeq 250 \mathrm{~K}$, with reduced scattering intensity. This process becomes complicated due to the presence of spin-orbital coupling in the host matrix of $\mathrm{CaRuO}_{3}$ .[82] Perhaps, this can be one of the reasons that the quasi-continuum persists to such a high temperature.[83] Because of their distinct temperature dependencies, these phenomena are considered magnetic. [81, 84]

The experimental results, manifested by the MACS data in Figure 3.8 af, are confirmed by independent measurement on cold spectrometer SPINS. A set of twodimensional inelastic scans at $\mathrm{Q}=1.15 \AA^{-1}$ at different temperatures are plotted in Figure 3.8h. The Q-value for the SPINS measurements were intentionally selected to avoid the inelastic peak position and thus, obtain a more realistic estimation of the spectral weight distribution to the background as functions of temperature and energy. Measurement at low temperature, $\mathrm{T}=3 \mathrm{~K}$, confirms the gapped nature of the localized excitation. As temperature increases, the localized excitation gradually melts and the spectral weight is transferred to low energy. It is as if the dimer pairs break and the freely fluctuating spins occupy the low energy spectrum between the singlet ground state and the triplet excited state in a continuous fashion, thus making it a gapless continuum. At higher temperature, $\mathrm{T} \geq 80 \mathrm{~K}$, the spectral weight also relocates to higher energies. This observation is consistent with the neutron data in Figure3.8 $\mathrm{d}-\mathrm{f}$, where significant scattering is observed at $\mathrm{T} \simeq 80 \mathrm{~K}$. Above $\mathrm{T}>80$ 
$\mathrm{K}$ (also comparable to the peak value of the broad fluctuation spectrum, $7.8 \mathrm{meV}$ ), thermal fluctuation starts dominating the spin fluctuations. At $\mathrm{T}=250 \mathrm{~K}$, it becomes very weak. Measurements in applied magnetic field of $\mathrm{H}=10 \mathrm{~T}$ did not reveal any field dependence of the inelastic properties of the system (see Figure 3.5).

\subsection{Heat Capacity Measurements}

We have performed heat capacity measurements on the substitution coefficient $\mathrm{x}=$ 0.17 sample in applied fields of $\mathrm{H}=0$ and $5 \mathrm{~T}$ utilizing the QD PPMS with a base temperature of $\mathrm{T}=1.8 \mathrm{~K}$. The background corrected data are shown in Figure 3.9. In the heat capacity plots, no sharp cusp indicating transition to a magnetic ordered state is observed. Also noticeable is the absence of magnetic field dependence of the heat capacity up to $\mathrm{H}=5 \mathrm{~T}$. Given the fact that the magnetic gap arises at $\mathrm{E}=5.9$ $\mathrm{meV}$, which is equivalent to $\mathrm{H} \simeq 40 \mathrm{~T}$ for spin- $1 / 2$, it is no surprise that heat capacity is independent of field up to $\mathrm{H}=5 \mathrm{~T}$. Also, the lack of any upturn in the heat capacity data at low temperature suggest the absence of the nuclear Schottky-type effect or the cluster spin glass behavior. 


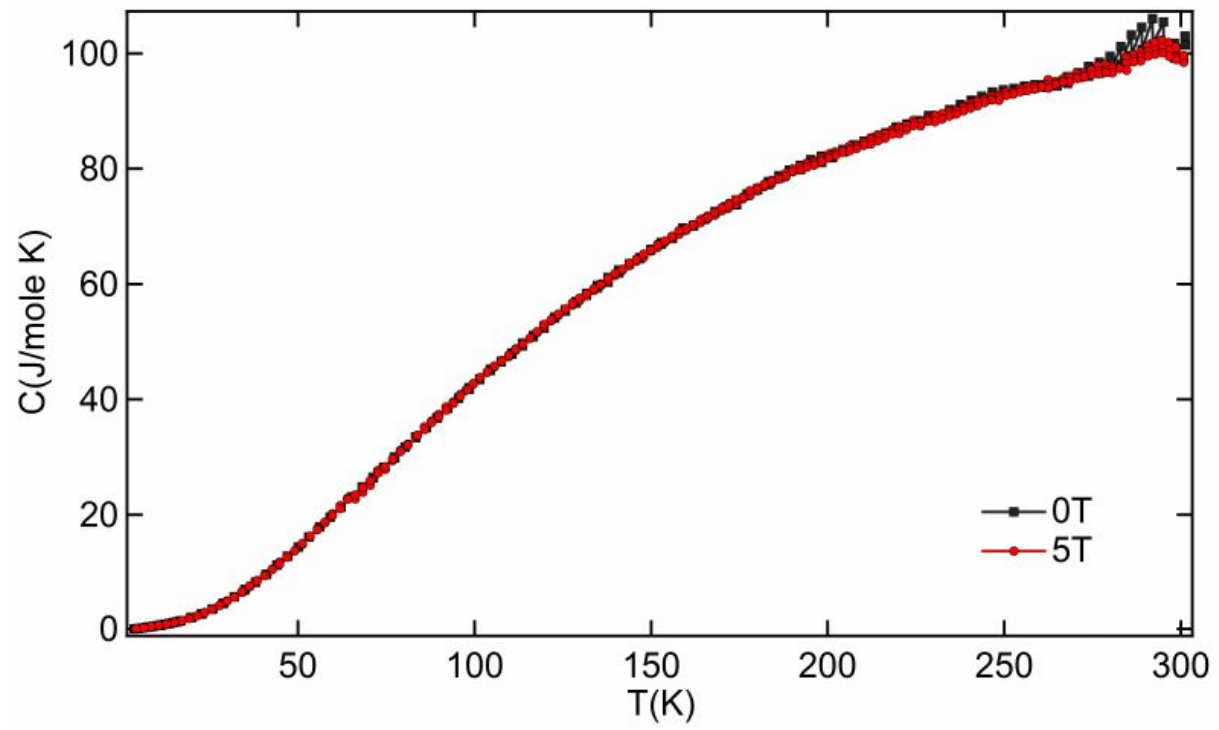

Figure 3.9: Heat capacity data of $\mathrm{Ca}\left(\mathrm{Co}_{0.17} \mathrm{Ru}_{0.83}\right) \mathrm{O}_{3}$. Heat capacity measurements performed on substitution coefficient $\mathrm{x}=0.17$ in applied magnetic field using a $\mathrm{QD}$ PPMS with a base temperature of $\mathrm{T}=1.8 \mathrm{~K}$. No signature of a transition to the ordered magnetic state is observed in the heat capacity data. 


\subsection{Results and Discussion}

Our experimental results provide new perspective to the study of quantum magnetism in spin-1/2 system for three reasons: first, the absence of any type of magnetic order rules out the ordered dimer arrangement of artificial spin- $1 / 2$, ultimately responsible for the VBS state. Similarly, the disorder in the lattice would prohibit coherent spin fluctuation, hence, the VBL state. However, the dimer formation in the insulating composition of $\mathrm{Ca}\left(\mathrm{Co}_{x} \mathrm{Ru}_{1-x}\right) \mathrm{O}_{3}$ involves simultaneous superposition of nearest neighbor and further separated artificial spin-1/2, pointing in random directions, by virtue of disorder. The dimerdimer interaction exhibits gapped singlet-to-triplet transition. Second, the break away spins of dimer pairs at high temperature create freely fluctuating fractionalized spins that occupy large cross section of the energy-momentum space. We also note that the fluctuation spectrum persists to much lower energy, as found in the ac susceptibility measurements where the dynamic susceptibility exhibits stronger frequency-dependence at higher temperature. Thus, the localized gapped excitation evolves into a gapless quasi-continuum spectrum as a function of temperature. $[81,20,85]$ These two phenomena combined with the absence of magnetic order in $\mathrm{Ca}\left(\mathrm{Co}_{x} \mathrm{Ru}_{1-x}\right) \mathrm{O}_{3}$, where $0.15<\mathrm{x}<0.2$, qualify to be a new quantum magnetic state, which depicts remarkable similarity to the resonant valence bond state. Additionally, this quasi-continuum spectrum persists to very high temperature, $\mathrm{T} \simeq 250 \mathrm{~K}$. The persistence of quasi-continuum to such an unusually high temperature extends the investigation to the semiclassical regime, which is a new frontier in the study of the quantum-mechanical properties in magnetic systems. Third and most important, we successfully demonstrate that a combination of disorder and the non-frustrated lattice can provide a new platform for future researches on quantum magnetism via the creation of local artificial spin-1/2. 


\section{Chapter 4}

\section{Spin Correlation in $\mathrm{EuMn}_{2} \mathrm{As}_{2}$}

122 pnictides have been explored for investigation of the interplay and coexistance of long-range magnetic order and superconductivity.[32, 33, 34, 35] One of the compunds that has been explored is $\mathrm{EuFe}_{2} \mathrm{As}_{2}$, which through substitutions at $\mathrm{Eu}, \mathrm{Fe}$ or As site and application of pressure has been seen to supress AFM ordering and give way to superconductivity that coexists with ordered $E u^{2+}$ moments. Similar to $\mathrm{EuFe}_{2} \mathrm{As}_{2}$, we have explored the 122-type pnictide $\mathrm{EuMn}_{2} \mathrm{As}_{2}$. Instead of haveing tetragonal structure like other 122 pnictides, $\mathrm{EuMn}_{2} \mathrm{As}_{2}$ crystallizes in trigonal $\mathrm{CaAl}_{2} \mathrm{Si}_{2}$-type structure (Space group P3m1).[50] The compound is shown to have 2 different magnetic transition. One transition at $14 \mathrm{~K}$ is associated with the $\mathrm{Eu}$ ordering. The other transition at 135 K. [51] However, the underlying reason for these transition has not been completely explored. Here, we performed extensive neutron scattering measurements to show the nature of the transition and have modeled the neutron scattering data to describe the nature of magnetic moment ordering. 


\subsection{Structure of $\mathrm{EuMn}_{2} \mathrm{As}_{2}$}

122-pnictides usually crystalize in $\mathrm{ThCr}_{2} \mathrm{Si}_{2}$-type tetragonal structure [8]. EuMn $\mathrm{As}_{2}$ is an anomaly to this hierarchical crystal symmetry. It crystallizes in trigonal $\mathrm{CaAl}_{2} \mathrm{Si}_{2}{ }^{-}$ type structure in the space group P-3m1 with lattice constants of $\mathrm{a}=\mathrm{b}=4.23 \AA$ and $\mathrm{c}=7.263 \AA[86,50]$. Careful analysis of the lattice structure reveals an interesting combination of magnetic ion arrangements in $\mathrm{EuMn}_{2} \mathrm{As}_{2}$, see Fig. 4.1, where Eu ions, $\mathrm{S}=7 / 2$, occupy the trigonal sites that are at the center of honeycomb subcell of $\mathrm{Mn}$ ions with $\mathrm{S}=5 / 2[51]$.

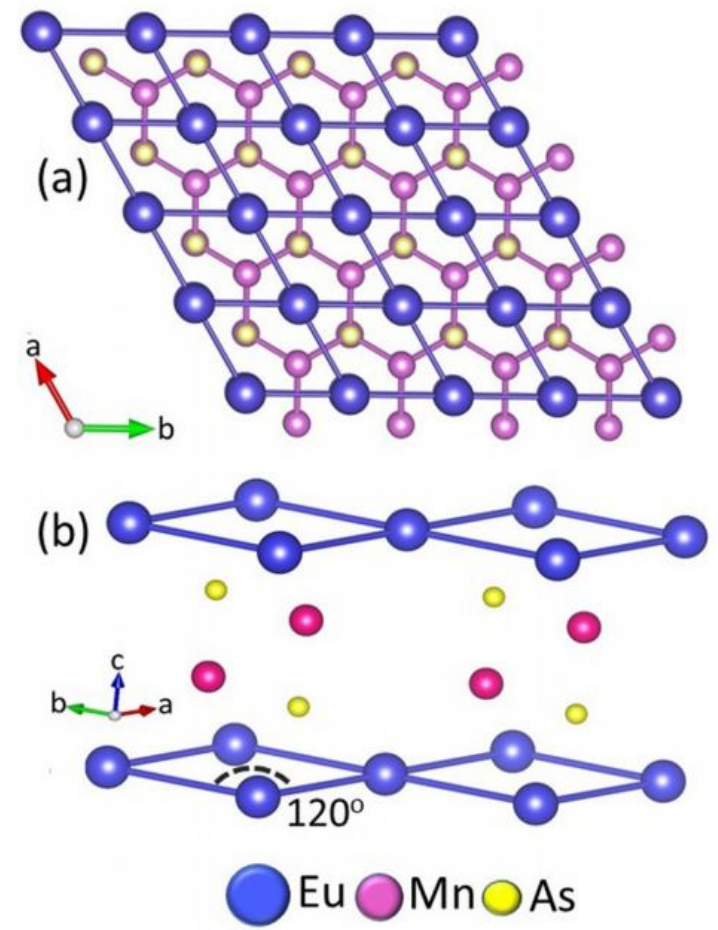

Figure 4.1: Crystal structure of $\mathrm{EuMn}_{2} \mathrm{As}_{2}$. (a) Mn ions arrange themselves in a twodimensional honeycomb pattern [51]. Blue, purple, and yellow spheres represent Eu, $\mathrm{Mn}$, and As ions. (b) $\mathrm{CaAl}_{2} \mathrm{As}_{2}$-type trigonal crystal structure of EuMn2As2(space group $\mathrm{P} 3 \mathrm{~m} 1)$. Eu ions arrange themselves on the vertices of trigonal lattice. 


\subsection{Neutron Scattering Measurements}

Neutron-scattering measurements were performed on $10 \mathrm{mg}$ flux grown thin rectangular shape single crystal of $E u M n_{2} A s_{2}$, dimension $3 \mathrm{~mm}$ (length) $\times 2 \mathrm{~mm}$ (width) $\times$ $1.25 \mathrm{~mm}$ (thickness), at the thermal Triple Axis Spectrometer, TRIAX, at the University of Missouri Research Reactor (MURR). Elastic measurements were performed at the fixed final energy of $14.7 \mathrm{meV}$ using PG (pyrolitic graphite) monochromator. The measurements on TRIAX employed a flat pyrolytic graphite (PG) analyzer with collimator sequence of PG filter-60 -60 -Sample-40 - PG filter-40 . Single crystal sample was mounted at the end of the cold finger of a closed cycle refrigerator with a base temperature of $T \sim 5 K$. Measurements were performed with the crystal oriented in the (HOL) scattering plane. Here $\mathrm{H}$ and $\mathrm{L}$ represent reciprocal lattice units of $4 \pi / \sqrt{3} a$ and $2 \pi / c$, respectively.

\subsubsection{Elastic Neutron Scattering Measurements}

Single-crystal measurements allow for a detailed examination of the intensities and magnetic scattering pattern, which reveals the nature of spin correlations that are not possible to obtain from magnetic and thermodynamic measurements. Elastic scans were obtained along both $\mathrm{H}$ and L-crystallographic directions, as shown schematically in Fig. 4.2(a) and Fig. 4.2(b). In Fig. 4.4(a) and Fig. 4.4(b), we plot background corrected elastic representative scans at $\mathrm{T}=6 \mathrm{~K}$ and $300 \mathrm{~K}$ across the [001] nuclear Bragg peak in $\mathrm{H}$ and L directions. As the sample is cooled to low temperature, resolution-limited enhancement of nuclear Bragg peak becomes apparent. Neutron-scattering data is well described by the Gaussian line shape. The additional scattering, which is arising due to magnetic correlation in the sample, indicates the development of commensurate long-range magnetic order in the system. Interestingly, elastic measurement across [001/2] reciprocal lattice vector also shows the develop- 
ment of resolution-limited magnetic peak as temperature is reduced to $\mathrm{T}=6 \mathrm{~K}$, see Fig. 4.4(c) and Fig. 4.4(d). It suggests that the ground-state magnetic configuration is anti-ferromagnetic in nature. Low-temperature elastic measurements depict magnetic Bragg peaks at the positions $\mathrm{h}+\mathrm{k}+\mathrm{l}=(\mathrm{n}+1) / 2$ [shown in 4.2(a) and $4.2(\mathrm{~b})]$.
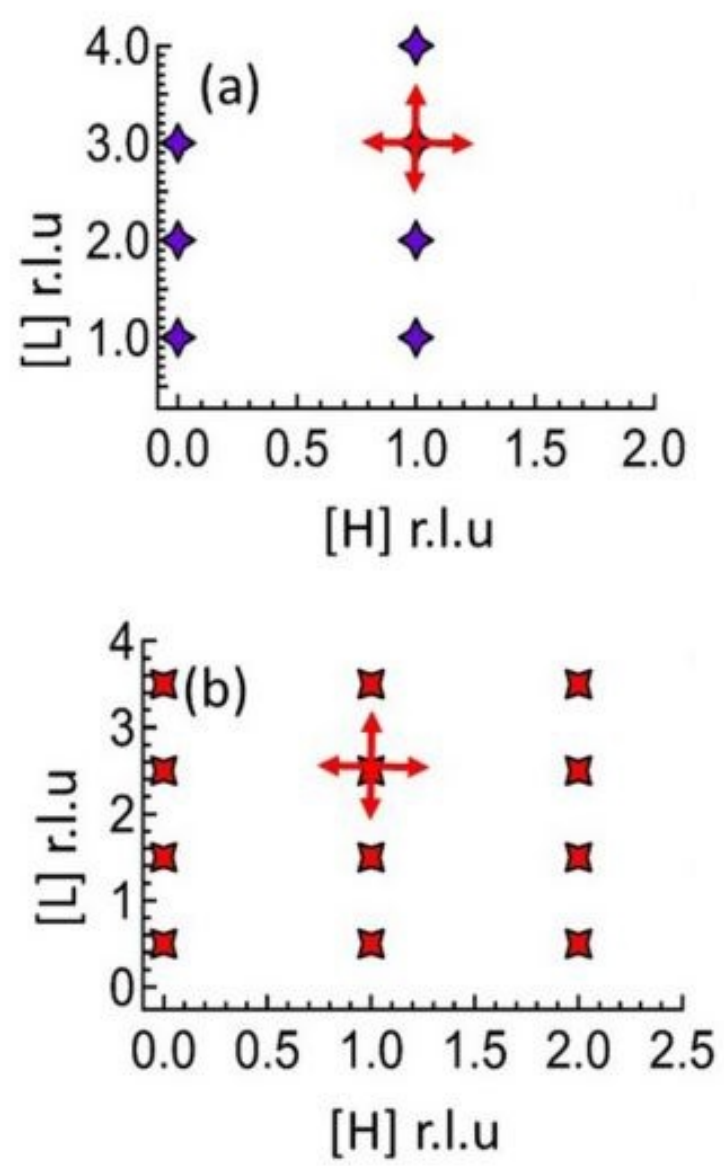

Figure 4.2: (a), (b) Schematic description of elastic scans along H and L directions across several Brillouin zones. 


\subsubsection{Absorption Correction for $\mathrm{Eu}$}

Since $\mathrm{Eu}$ is a strong absorber of neutron, absorption corrections were made to the elastic neutron data. The absorption correction was performed by multiplying the experimental data with the inverse of transmission coefficient A, given by $A=\frac{1}{V} \exp (\mu t) d V$, where $\mu$ is neutron absorption coefficient and $\mathrm{t}$ is the thickness of the sample [25]. For $\mathrm{Eu}, \mu$ is $\simeq 140 \mathrm{~cm}^{1}$ at $\mathrm{E}=14.7 \mathrm{meV}$ [87]. $\mathrm{EuMn}_{2} \mathrm{As}_{2}$ single crystal, used in this study, was a very thin sample (thickness $\simeq 1.25 \mathrm{~mm}$ ) with slab geometry. For such a geometry, coefficient A in the case of maximum absorption or negligible transmission is given by

$$
A=\frac{\sin (\theta-\phi)}{\mu\{\sin (\theta-\phi)+\sin (\theta+\phi)\}}
$$

where $\theta$ is the sample angle and $\phi$ is the angle between sample surface and the scattering plane. In a particular situation when the neutron beam is parallel to the scattering plane of the crystal such that the absorption is higher, i.e., $\phi=0$, the coefficient A simplifies to

$$
A=[1-\exp (2 \mu t \cdot \operatorname{cosec} \theta)] / 2 \mu
$$

Absorption correction to the diffraction data did not make any significant difference for such a thin sample. Correction to the estimated intensities due to Eu absorption were found to be within the statistical error margin.

\subsubsection{Order Parameter Measurements}

The observation of long-range antiferromagnetic order at low temperature in $\mathrm{EuMn}_{2} \mathrm{As}_{2}$ is consistent with a previous report, based on magnetic and thermodynamic measurements [51]. However, direct evidence to magnetic transition at $\mathrm{T} \sim 140 \mathrm{~K}$ is lacking. Elastic scans, shown in Fig. 4.4(c)-4.4(f), hint at the existence of two different mag- 
netic correlations; one commensurate with the lattice structure and the other with a magnetic unit cell twice that of the lattice unit cell. To know if the two magnetic correlations manifest different temperature dependencies, we have performed the measurement of order parameters as a function of temperature at magnetic Bragg peaks of [001] and [001/2]. As shown in Fig. 4.3, two entirely different temperature dependencies are manifested by the system. The order parameter data are fitted with the power law equation

$$
I \propto\left(1-T / T_{N}\right)^{2 \beta}
$$

To accurately estimate the magnetic transition temperature and the critical exponents that can provide important information about the nature of the phase transition. While low-temperature transition is found to occur at $\mathrm{T}=14.4 \mathrm{~K}$ and manifest a critical exponent of $2 \beta=0.42$, the higher temperature transition occurs at $\mathrm{T}=135$ $\mathrm{K}$ with a critical exponent of $2 \beta=0.40$. The value of exponent $\beta$ also suggests threedimensional interaction in the ordering regime [88]. Corresponding ordered moments for integer and half-integer diffraction peaks, at $\mathrm{T}=6 \mathrm{~K}$, are determined to be $2.8(3)$ $\mu_{B}$ and 1.6(4) $\mu_{B}$, respectively, which indicates the dominance of Mn-spin at higher temperature and Eu-spin at lower temperature. 

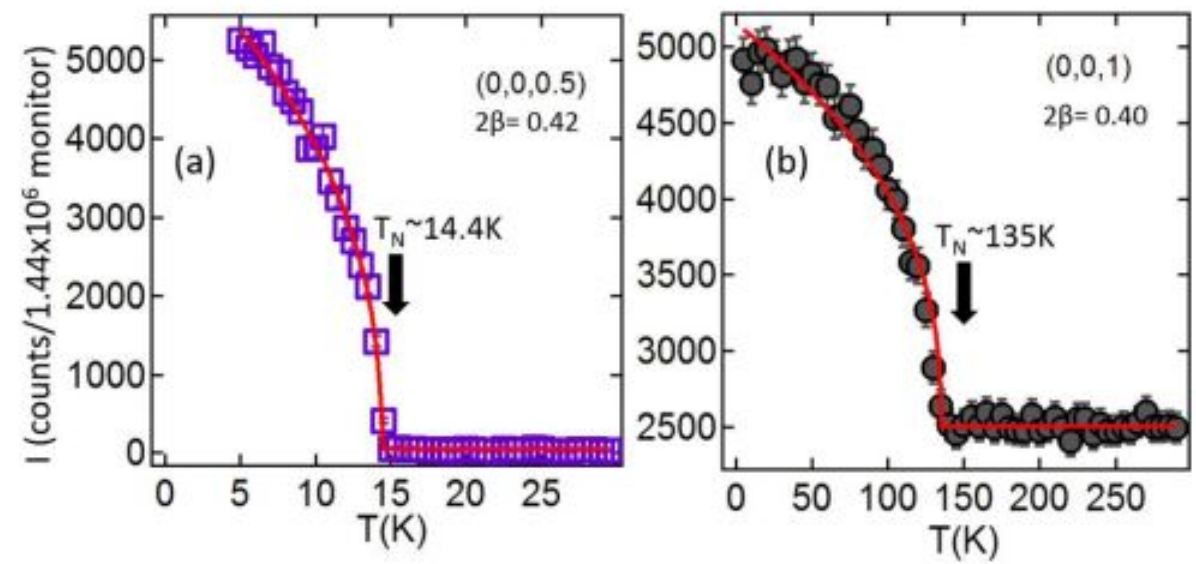

Figure 4.3: (a) Order parameter measurement performed at [001/2] peak position. Experimental data is fitted with a power law (see text for detail) to obtain information about magnetic transition temperature. The system tends to develop magnetic order below $\mathrm{T}=14.38 \mathrm{~K}$, with critical exponent $2 \beta=0.42$. (b) Measurement of order parameter at nuclear peak position [001] reveals an entirely different magnetic ordered regime, as manifested by the transition temperature of $\mathrm{T}=135 \mathrm{~K}$. However, the critical exponent $2 \beta$ is comparable, $\sim 0.4$, to that observed at [001/2]. Error bars represent one standard deviation. 


\subsubsection{Numerical Modeling of Elastic Data}

Clearly, $\mathrm{EuMn}_{2} \mathrm{As}_{2}$ exhibit two different magnetic ordered regimes. The transition at higher temperature seems to be arising due to the long-range correlation of Mnspins, which persists to the lowest measurement temperature. A new magnetic order, however, develops below $\mathrm{T}=14.4 \mathrm{~K}$, which is most likely associated to $\mathrm{Eu}$ spins. Magnetic orders in the two sublattices of $\mathrm{Mn}$ and $\mathrm{Eu}$ ions are, apparently, cooperative in nature. Insights about the nature of spin correlations in Mn and Eu sublattices are gained from numerical modeling of the elastic scattering data. For this purpose, elastic measurement was performed across several Brillouin zones, see 4.5(a). The experimentally observed structure factor, estimated from the Gaussian fit of the elastic data, are compared with the numerically calculated structure factor for model spin configurations, see Tables I and II. Structure factor is calculated using

$$
F_{M}=\Sigma_{j} S_{\perp j} P_{j} e^{i Q r_{j}} e^{W_{j}}
$$

[89], where $S_{\perp}=\hat{Q} \times(S \times \hat{Q})$ is the spin component perpendicular to the $\mathrm{Q}, p=$ $\left(\frac{\gamma r_{0}}{2}\right) g f(Q),\left(\frac{\gamma r_{0}}{2}\right)=0.26951012 \mathrm{~cm}, \mathrm{~g}$ is the Lande splitting factor andwas taken to be

$\mathrm{g}=2, \mathrm{f}(\mathrm{Q})$ is the magnetic form factor, and $e^{W_{j}}$ is the Debye-Waller factor and was taken to be 1 . 

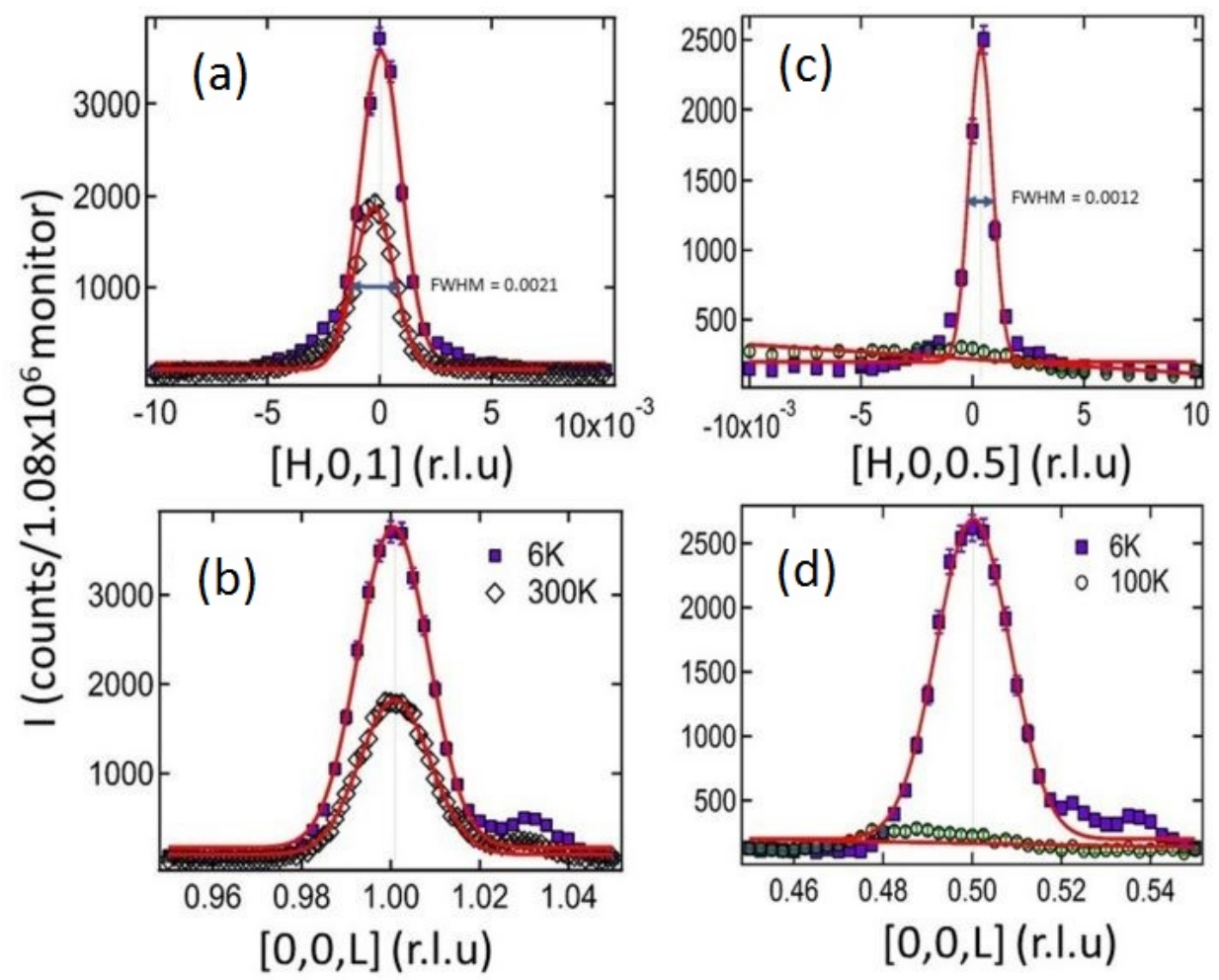

Figure 4.4: (a), (b) Scans along H and L directions across [001] nuclear peak at 6 $\mathrm{K}$ and $300 \mathrm{~K}$, respectively. Each data point is counted for about 90 seconds. Strong enhancement in scattering intensity is observed as sample is cooled to low temperature of $\mathrm{T}=6 \mathrm{~K}$. Experimental data are well described by Gaussian line shape. Elastic scans are instrument resolution limited, thus suggesting the long-range nature of spin correlation. (c), (d) Elastic scans across [001/2] peak, along $\mathrm{H}$ and L directions, at $\sim 6 \mathrm{~K}$ and $100 \mathrm{~K}$ are shown here. Unlike the nuclear peaks, the peak completely disappears at higher temperature. Once again, the peaks are instrument resolution limited. Error bars represent one standard deviation. 
Table 4.1: Experimentally estimated and calculated intensities at half-integer magnetic wave vectors at $\mathrm{T}=6 \mathrm{~K}$. As shown in Fig. 4.5(a), the peak intensity at $[003 / 2]$ position is spurious in nature

\begin{tabular}{ccc}
\hline$[$ HKL $]$ & Experimental intensity & Calculated intensity \\
\hline$[001 / 2]$ & 2490 & 2469 \\
{$[003 / 2]$} & 4130 & 2126 \\
{$[005 / 2]$} & 1470 & 1604 \\
{$[007 / 2]$} & 1420 & 1089 \\
{$[101 / 2]$} & 657 & 717 \\
{$[103 / 2]$} & 357 & 290 \\
{$[105 / 2]$} & 287 & 325 \\
{$[107 / 2]$} & 446 & 332 \\
{$[205 / 2]$} & 80 & 120 \\
{$[207 / 2]$} & 62 & 86 \\
\hline
\end{tabular}

Table 4.2: Experimentally estimated and calculated intensities at integer magnetic wave vectors at $\mathrm{T}=6 \mathrm{~K}$.

\begin{tabular}{lcc}
\hline$[\mathrm{HKL}]$ & Experimental intensity & Calculated intensity \\
\hline$[001]$ & 1860 & 1818 \\
{$[002]$} & 3040 & 2843 \\
{$[003]$} & 912 & 1044 \\
{$[101]$} & 313 & 327 \\
{$[102]$} & 80 & 80 \\
{$[103]$} & 20 & 9 \\
{$[104]$} & 120 & 127
\end{tabular}

Numerical modeling was performed for various different scenarios. Best fit to the neutron diffraction data at integer positions is obtained for a spin configuration where $\mathrm{Mn}$ moments are arranged antiferromagnetically in the a-b plane and aligned ferromagnetically along the $\mathrm{c}$ axis. In this process, moments are rotated by $\theta=55^{0}$ with respect to the c axis and $\phi=12^{0}$ with respect to the a axis in the a-b plane, see Fig. 4.5(b). Lower transition-temperature magnetic phase, represented by diffraction peaks at half-integer positions, is well described by antiferromagnetic spin correlation of Eu spins along the $\mathrm{c}$ axis where moments are rotated by $\theta=55^{0}$, 

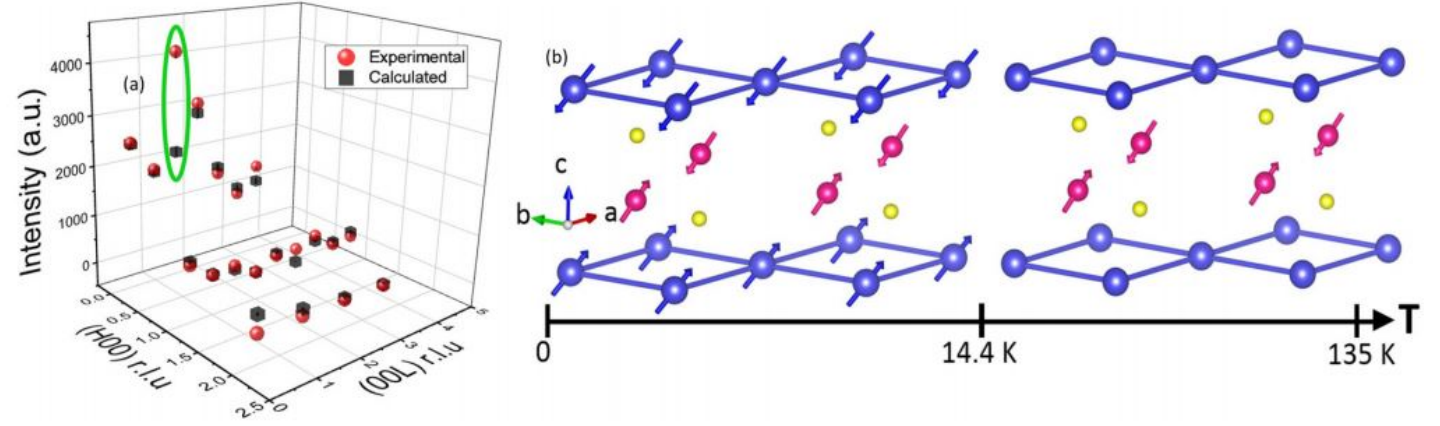

Figure 4.5: (a) Comparison of experimental, obtained at $\mathrm{T}=6 \mathrm{~K}$, and calculated intensities at magnetic Bragg peaks. Good agreement between experimental and calculated intensities is obtained for the proposed spin structure. Green ellipse, enclosing a data point and calculated intensity, highlights spurious scattering. Error bars represent one standard deviation. (b) Numerical modeling of neutron data reveals two different antiferromagnetic orders. Mn spins tend to develop antiferromagnetic correlation below $\mathrm{T}=135 \mathrm{~K}$ in the a-b plane where they are rotated by $\phi=12^{0}$ with respect to a axis and $\theta=55^{0}$ with respect to c axis. As temperature is reduced below $\mathrm{T}=14.4 \mathrm{~K}$, new magnetic order due to Eu spin antiferromagnetic correlation arises. Unlike Mn spins, Eu spins order along the c axis where they are rotated by $\phi=5^{0}$ in the a blane and by $\theta=55^{0}$ with respect to $\mathrm{c}$ axis. 


\subsection{Results and Discussion}

We have investigated the nature of spin correlation and magnetic transition in single crystal EuMn $\mathrm{As}_{2}$. The transition at low temperature, $\mathrm{T}_{N}=14.4 \mathrm{~K}$, is somewhat consistent with previous magnetic and thermodynamic measurements. But the second transition at higher temperature, $\mathrm{T}_{N}=135 \mathrm{~K}$, was not observed before. Many pnictide materials of lanthanide family are known to exhibit multiple magnetic transitions $[90,91]$. Numerical modeling of the elastic neutron data reveals distinct long-range antiferromagnetic correlation of $\mathrm{Eu}$ and $\mathrm{Mn}$ spins. While Mn spins are antiferromagnetically aligned in the a-b plane, Eu spins develop AFM order along the c axis. However, in both cases, moments are significantly rotated from the $\mathrm{c}$ axis and aligned closely to the diagonal axis. The modeled spin configuration appears to be different from the previously proposed Ising configuration in this system or in $\mathrm{SrMn}_{2} \mathrm{As}_{2}$ where Das et al. used neutron diffraction on single crystal sample to determine the collinear magnetic moment correlation of Mn-ions[47, 51]. Clearly, more research works are needed to understand this discrepancy. Although, $\mathrm{SrMn}_{2} \mathrm{As}_{2}$ and $\mathrm{EuMn}_{2} \mathrm{As}_{2}$ are structurally similar, the underlying physics can be quite complex in $\mathrm{EuMn}_{2} \mathrm{As}_{2}$ due to the presence of two magnetic ions on structural motif. Neutron-based study of $\mathrm{EuMn}_{2} \mathrm{As}_{2}$ will help us in developing a comprehensive understanding of this complex behavior. Future research works, especially inelastic neutron scattering measurements on large single crystal of $\mathrm{EuMn}_{2} \mathrm{As}_{2}$, are highly desirable. Understanding the interaction between Eu and Mn spins as well as the valence fluctuation of Eu ions can shed light on the cooperative nature of spin configurations and their role in the anomalous insulating electrical properties in this system. 


\section{Chapter 5}

\section{Magnetism in Artificial Honeycomb Lattice of Py}

The interplay between magnetic and thermodynamic characteristics often dictates the nature of phase transition in a magnetic material. Theoretical researches have shown that an artificial magnetic honeycomb lattice can undergo a series of thermodynamic phase transitions as a function of reducing temperature from a paramagnetic phase, consisting of the distribution of two-in \& one-out (or vice-versa) and all-in or all-out moment arrangements, to a short-range ordered spin ice state $[92,93]$. For further reduction in temperature, the system tends to develop a magnetic charge ordered state, which is described by the random distribution of chiral vortex loops. At much lower temperature, a honeycomb lattice is predicted to develop a novel ground state of spin solid order, described by the periodic arrangements of the vortex magnetic loops of opposite chiralities[94]. Each magnetic phase transition reduces the overall entropy of the system. The transition to the spin solid ground state is expected to be truly thermodynamic in nature, with zero entropy and magnetization at low temperature .

Detailed understanding of a magnetic material or a particular physical or magnetic state requires the knowledge of electrical properties as well as magnetic properties. For 
this purpose, we have done through study of the electrical properties in the system. By analyzing the data for electrical resistivity, we have found the electrical dimensionality of the system. We have also done non-linear susceptibility scaling analysis of the system by taking intensive magnetic measurements. These measurements provide an insight into the nature of magnetic phase transition in the system.
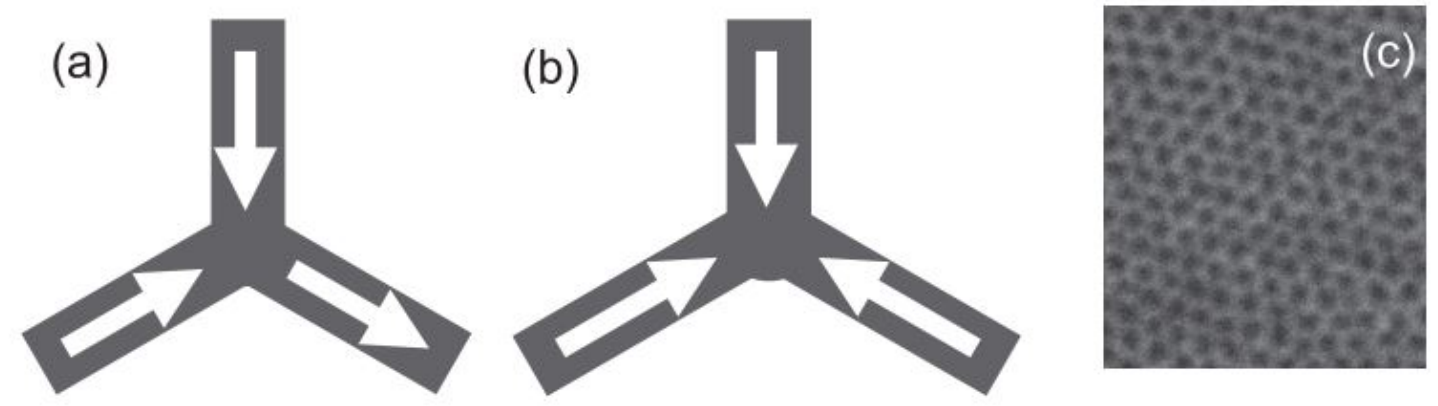

Figure 5.1: (Moment configuration. in Artificial Honeycomb Lattice (a) Schematic of two-in and 1-out moment configurations on a honeycomb lattice vertex. Here, two moments are pointing to the vertex and one point is pointing away from the vertex. (b) All-in moment configuration where all three moments are pointing to the vertex. (c) The scanning electron micrograph of a typical artificial honeycomb lattice, fabricated using the diblock template synthesis method. 


\subsection{Sample Characterization}

To study the physics in the system, it is important for the sample to be a well ordered honeycomb. To check the order in the sample, we performed AFM measurements before and after etching the diblock template. AFM measurements were performed using Digital Instruments AFM. The measurements were taken in tapping mode, where the tip is moved at the resonance frequency of $\sim 170 \mathrm{kHz}$ to avoid continuous contact with the surface. This reduces the lateral forces and drag on the AFM tip. The tip was aluminium coated Silicon tip with diameter of $\sim 8 \mathrm{~nm}$. We also performed SEM measurements to characterize the sample after depositing magnetic material.

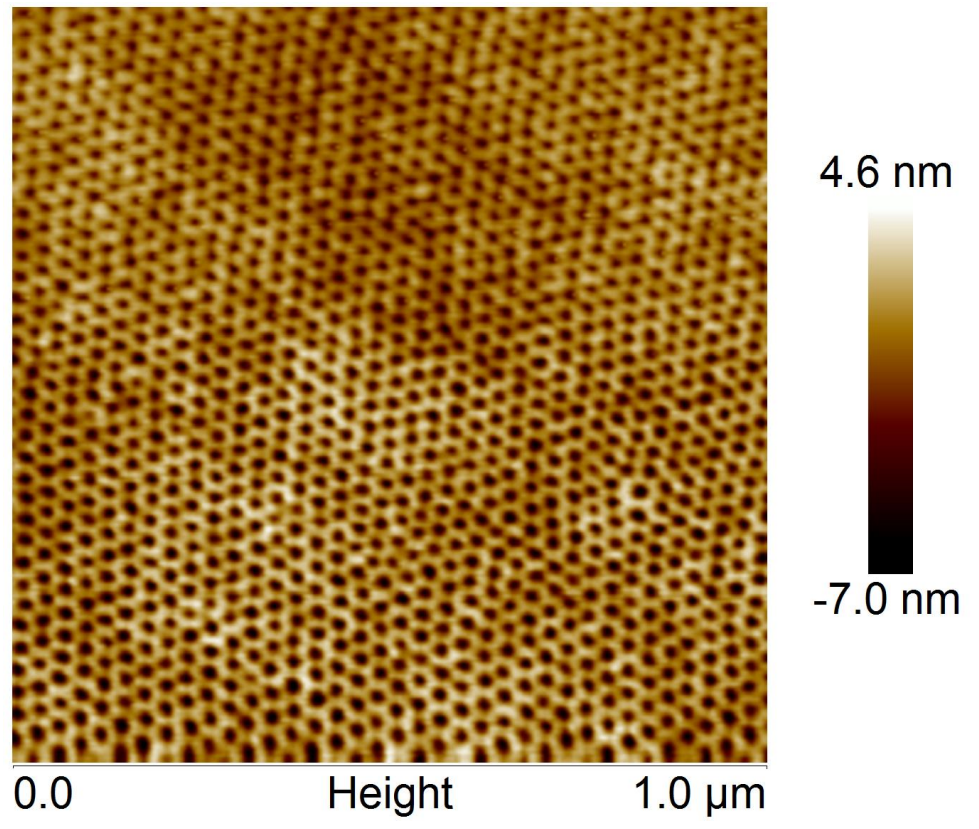

Figure 5.2: AMF image of honeycomb structure after RIE 


\section{$5.2 \quad$ Electrical Measurements}

We plot the normalized electrical resistivity as a function of temperature in Fig. 5.3(a). There are several different temperature regimes, indicative of different magnetic phases in the system.[92, 95] Previously, detailed small angle neutron scattering (SANS) and reflectometry measurements provided insight into magnetic correlation in different temperature regimes in this system. As described schematically in Fig. 5.3(b), the system undergoes a transition from the paramagnetic phase, where the magnetization across the lattice is described by the random distribution of 2 -in and 1-out (or vice-versa) and all-in or all-out moment arrangements on the honeycomb vertices to the short-range ordered spin ice phase, primarily involving 2 -in and 1-out (or vice-versa) configurations only. Here, 2-in and 1-out refer to a peculiar configuration of two moments, aligned along the honeycomb lattice elements due to the shape and magneto crystalline anisotropy, one pointing to the vertex and the other pointing away from the vertex in the system. Thus, it obeys the quasi ice rule. Similarly, all-in and all-out moment configurations indicate situations where all moments are either pointing to the vertex or pointing away from the vertex, respectively. As temperature is reduced further, the magnetization pattern changes to the magnetic charge ordered state, which consists of a random pair of vortex loops of opposite chiralities. At much lower temperature, $T \simeq 30 K$, the system is found to develop long range spatial correlation of spin solid order, manifested by the ordered arrangements of the vortex loops of opposite chiralities of magnetic moments. We observe that the electrical resistivity exhibits a very sharp enhancement of more than two orders of magnitude, compared to the value at $T \sim 40 K$, in the spin solid regime. The artificial honeycomb lattice exhibits strong insulating characteristics in the long range ordered phase. The spin solid phase is manifested by the vortex loops of opposite chiralities.

The electrical insulating behavior in the spin solid phase at low temperature is unusually strong. This is a very surprising observation. After all, the lattice struc82 
ture parameters remain unchanged and the only change occurs in the form of the rearrangement of the magnetization pattern. To understand this, we have performed dimensionality analysis of low temperature electric data using the Aslamozov-Larkins (AL) model. [96] The AL model is often used to deduce the electrical dimensionality of a material, especially type-II superconductors where the underlying physics is dominated by the pairs of magnetic vortex and anti-vortex circulations that give rise to additional resistance.[97, 98] Similar magnetic vortices are the building blocks of the spin order state. The AL model correlates conductivity with a characteristic parameter $\mathrm{t}$ vis the following relation:

$$
\sigma=(a+b T)^{-1}+c t^{-(\epsilon+1)}
$$

where $\mathrm{a}, \mathrm{b}$, and care the constants and $\mathrm{t}$ is given by

$$
t=\left(1-T / T_{0}\right)
$$

Here, $T_{0}$ is the onset to the spin solid phase, $T_{0}=33 \mathrm{~K}$. The fitting parameter $\epsilon$ is related to the electrical dimensionality d by $d=4-2 \epsilon$. We fit the low temperature conductivity data using the AL model, as shown in Fig. 5.4. The estimated electrical dimensional value is found to be $d=0.6(0.04)$, which is much smaller than the surface dimensionality $(D=2)$. This suggests that the electrical properties do not follow the typical surface transport behavior at low temperature. If we use the same model to fit the data in other temperature regimes, then the estimated dimensionality is found to be $d=2.06(0.14)$, which is close to the full lattice dimensional value of 2 (see the inset in Fig. 5.4). The two dimensional character is preserved across the entire temperature range of the measurement, except in the spin solid regime where it exhibits fractional dimensionality.

In summary, we have observed two interesting electrical properties: first, the sys83 
tem exhibits a strong insulating characteristic as soon as it enters in the spin solid phase; second, the electrical transport follows a fractional dimension at low temperature, which is even smaller than $d=1$. In general, a bulk material (of electrical dimensionality three) manifests smaller resistance, compared to the lower dimensional system, e.g., two-dimensional system. As the dimensionality reduces, the electrical resistance tends to increase.[99] If the honeycomb lattice follows the trend, then the electrical dimensionality of $d=0.6$ at low temperature should exhibit a much larger resistance, compared to the full two dimensional value at higher temperature.This suggests that the electrical transport is non-surface-like phenomenon, perhaps represents a path-like behavior. The experimental observation is consistent with this qualitative explanation.
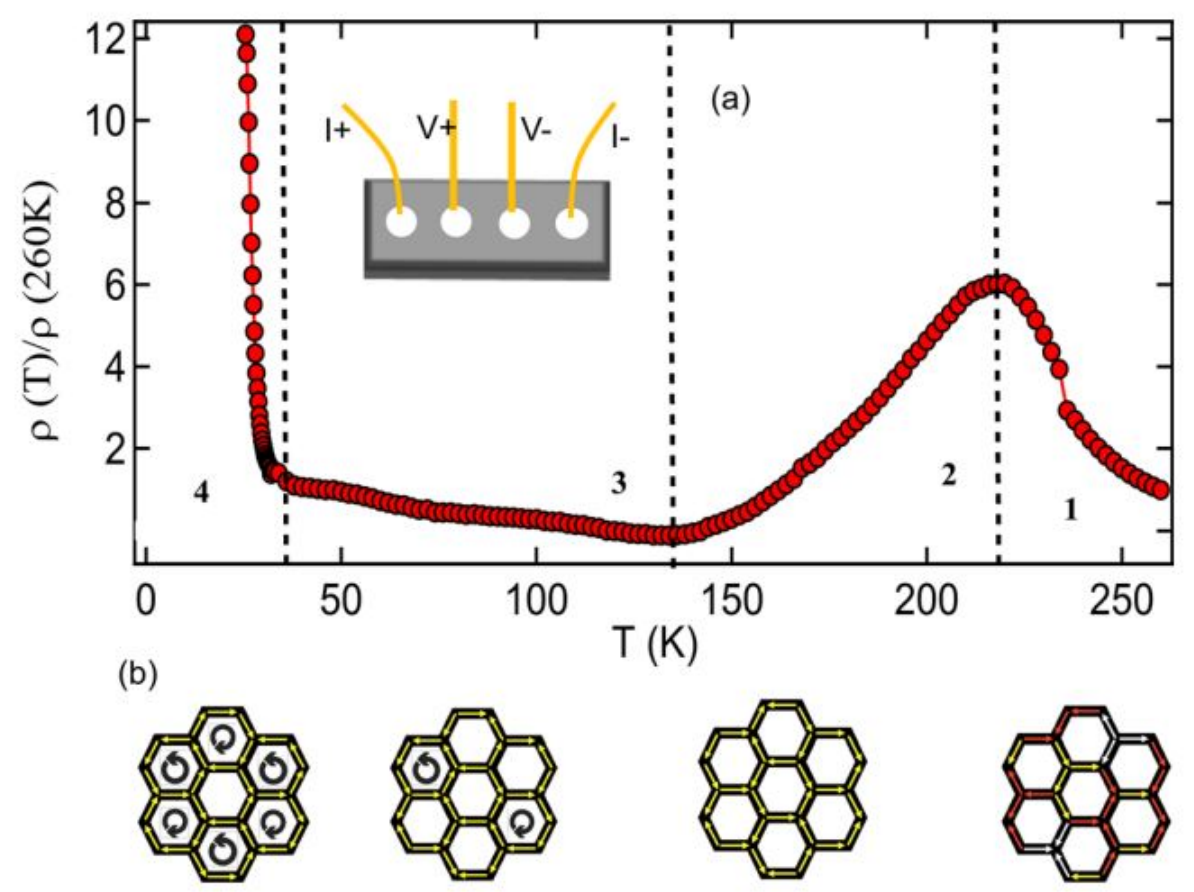

Figure 5.3: Resistivity vs Temperature measurement done using four probe method. 


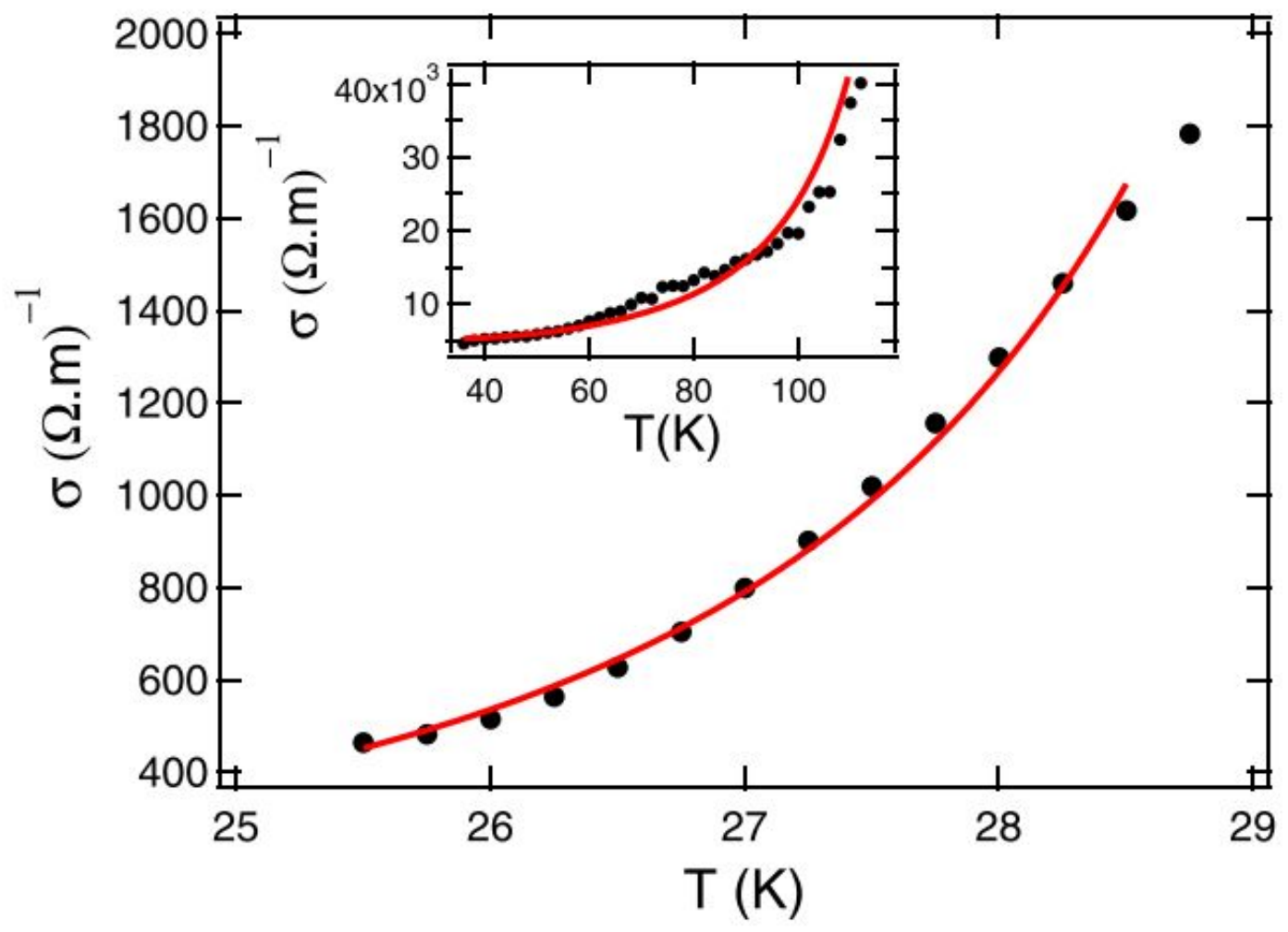

Figure 5.4: Conductivity vs Temperature data fitted with AL model 


\subsection{Magnetization Measurements}

Previously, we had observed the transition to the novel zero entropy spin solid state as temperature was reduced in the $2 \mathrm{D}$ honeycomb lattice to $\sim 7 \mathrm{~K}$. [95] As per the theoritical prediction, the system will go through several magnetic phase transition where the overall entropy of the system reduces. The transition to the spin solid ground state is expected to be truly thermodynamic in nature, with zero entropy and magnetization at low temperature.[59, 100, 101] Analysis of non linear susceptibility provides an ideal method to test the equilibrium nature of a magnetic phase transition. [102, 103, 104] An equilibrium phase transition is manifested by the scaling of nonlinear susceptibilities where the static critical exponents are related to each other via a conventional relation. To understand the equilibrium nature of the spin solid order, it is desirable to investigate the properties of nonlinear susceptibilities in artificial honeycomb lattice. For this purpose, we performed detailed magnetic measurements to perform the Non-linear susceptibility scaling analysis. We performed magnetization vs applied field at various temperatures. From these measurement non-linear susceptibility could be deducted for analysis.

In the case of an equilibrium phenomenon, the nonlinear susceptibilities exhibit a scaling behavior according to the single parameter, given by:

$$
\chi_{n 1}(T, H)=H^{2 / \delta} f\left(\tau^{(\gamma+\beta) / 2} / H\right)
$$

where $\tau=\left(T / T_{s}\right)-1, \gamma$ is the critical exponent describing the divergent nature of magnetic susceptibility as a function of temperature, and $\beta$ is the magnetic order parameter critical exponent. The determination of nonlinear susceptibility $\chi_{n 1}$ plays the key role in this exercise. The nonlinear susceptibilities are written as the higher 
order terms in following equations:

$$
\begin{gathered}
M / H(T)=\chi_{1}(T)-\chi_{3}(T) H^{2}+O\left(H^{4}\right) \\
=\chi_{1}(T)-a_{3}(T) \chi_{1}^{3} H^{2}+O\left(H^{2}\right) \\
\chi_{n 1}(T, H)=1-M(T, H) / \chi_{1} H
\end{gathered}
$$

where $\chi_{1}(T)$ is the linear susceptibility at temperature $\mathrm{T}, \chi_{3}(T)$ is the non linear susceptibility, coefficient $a_{3}=\chi_{3} /\left(\chi_{1}\right)^{3}$, and $\chi_{n 1}$ is the net nonlinear susceptibility.

Determination of the critical exponents $\gamma$ and $\beta$ depends on the asymptotic nature of the arbitrary scaling function $f(x)$, with the boundary conditions

$$
f(x)=\text { const as } x \rightarrow 0
$$

and

$$
f(x)=x^{-1 \gamma /(\gamma+\beta)} \text { as } x \rightarrow \infty .
$$

The nonlinear susceptibility $\chi_{n 1}(T, H)$ is expected to follow power-law depedence in both $T$ and $H$ with two independent static critical exponents $\gamma$ and $\delta$, respectively. The power-law dependencies are described by the following expressions:

$$
\begin{gathered}
\chi_{n 1}(T) \propto \tau^{|\gamma|}, \\
\chi_{n 1}\left(T \simeq T_{s}, H\right) \propto H^{2 / \delta}
\end{gathered}
$$

The two independent exponents $\gamma$ and $\delta$ are related to the magnetic order parameter critical exponent $\beta$ via the following scaling relation:

$$
|\delta|=1+|\gamma / \beta|
$$


The above scaling relation represents a robust test, arguably, of the true equilibrium phase transition in a magnetic system. Magnetization data on the newly designed artificial permalloy $\left(\mathrm{Ni}_{0.81} \mathrm{Fe}_{0.19}\right)$ honeycomb lattice were obtained in the field range of 10 - 1500 Oe using a commercial magnetometer. The sample was slowly cooled from $\mathrm{T}=350 \mathrm{~K}$ to the desired temperature before collecting the data. Extra care was taken in removing magnetic hysteresis in the superconducting magnet of the magnetometer by cycling the magnetic field in oscillatory mode several times at $\mathrm{T}=350 \mathrm{~K}$ before cooling to the measurement temperature. At each field, the system was allowed to sufficiently relax before collecting the data.

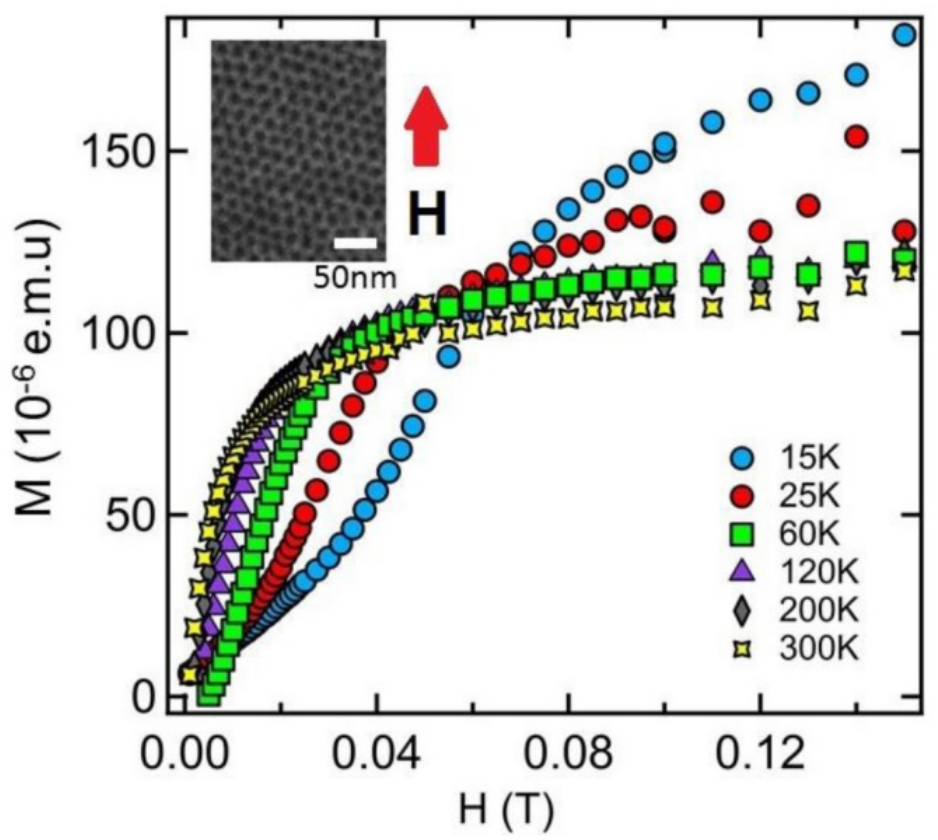

Figure 5.5: Here magnetization is plotted as a function of field at different temperatures. Magnetization data exhibits a crossover behavior in field. While the higher temperature susceptibility is stronger at low field, the magnetization at low temperature is larger above the crossover field $H \simeq 0.04-0.06 T$. Inset shows the scanning electron micrograph of a typical artificial honeycomb lattice of ultrasmall elements. Magnetic field was applied in-plane to the lattice.

In Fig. 5.5 we plot the MvsH data at a few characteristic temperatures. The total magnetization at higher temperature is stronger at low field. The trend reverses across 
the crossover field, which also varies with temperature. The linear susceptibility $\chi_{1}(T)$ at different temperatures were determined by fitting the $\mathrm{M}$ versus $\mathrm{H}$ curves at low fields. We have analyzed the first and second order term in the magnetization data. Beyond the second order term, the nonlinear susceptibility becomes much smaller to be of any quantitative importance. Therefore, Eq. (5.4) reduces to

$$
\chi_{3}(T, H) H^{2}=1-M(T, H) / \chi_{1} H
$$

Hence, $\chi_{n 1}(T, H)$ becomes

$$
\left(\chi_{3} / \chi_{1}\right)(T, H) H^{2}
$$

In Fig. 5.6 we have plotted net nonlinear susceptibilities $\chi_{n 1}(T, H)$ as a function of $H^{2}$ at different temperatures between $\mathrm{T}=10$ and $300 \mathrm{~K}$. The plot of nonlinear susceptibility reveals several very interesting behaviors in applied field. First, at low temperature, $T \simeq 25 K, \chi_{n 1}$ is negative for the entire field application range. The negative nonlinear susceptibility suggests that the higher order correction to the linear susceptibility is very strong. Surprisingly, negative $\chi_{n 1}$ is only observed below the spin solid phase transition. Second, the nonlinear susceptibility not only becomes positive above $T \simeq 30 K$, but also exhibits an unusual trend at low field. At low field, $\chi_{n 1}$ first decreases before manifesting a gradual enhancement as the applied field strength increases. Thus, the slope of the curve changes from negative (regime 1) in low field to positive (regime 2) in high field. Additionally, the slope of the curve also changes as a function of temperature at low field: from positive at $T \simeq 30 \mathrm{~K}$ to negative at $T \simeq 30 K$. We summarize these observations in plot of $\chi_{3}$ vs $T$ in different field regimes. In general, nonlinear correction to the susceptibility only changes in magnitude, not in sign. This is a puzzling behavior in artificial honeycomb lattice. The characteristic crossover field, separating the two distinct regimes, decreases as the measurement temperature increases. We also notice that the saturated value of 
$\chi_{n 1}$ increases as temperature increases. The net magnetization is expected to decrease as temperature increases. We also notice that the saturated value of $\chi_{n 1}$ increases as temperature increases. The net magnetization is expected to decrease as temperature reduces in artificial honeycomb lattice.

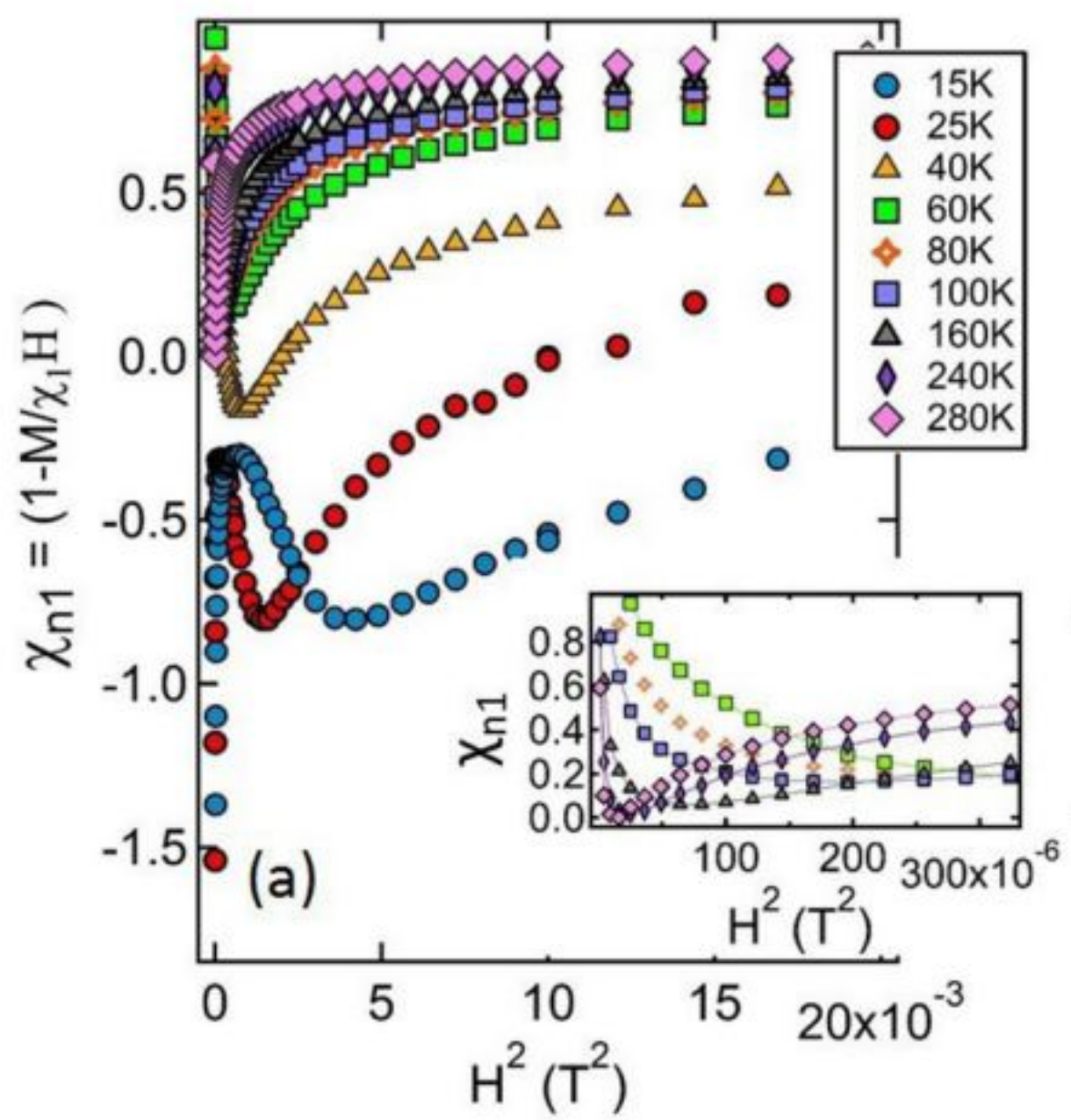

Figure 5.6: (a) $\chi_{n 1}$ is estimated using Eqs. (5.4)-(5.6)where $\chi_{1}$ is obtained from fitting MvsH plot at low field. Two features are immediately obvious in this figure: a change in the sign of overall nonlinear susceptibility across $T \simeq 30 \mathrm{~K}$ and a crossover regime in field and temperature. As shown in the inset of the figure, the slope of the curve changes from negative to positive at some field value. We call it characteristic crossover field, which increases as temperature decreases. 
First, we analyze the nonlinear susceptibility data above the characteristic field (in regime 2). Even in regime 2, the maximum value of the field up to which $\chi_{n 1}(T)$ is linear in $H^{2}$, decreases gradually as $T$ approaches $T_{s}$. It suggests that the higher order corrections in the net susceptibility is still significant. The linear portion of $\chi_{n 1}(T)$ at different temperatures are fitted with Eq. (5.5) to extract the coefficient $a_{3}(T)$.
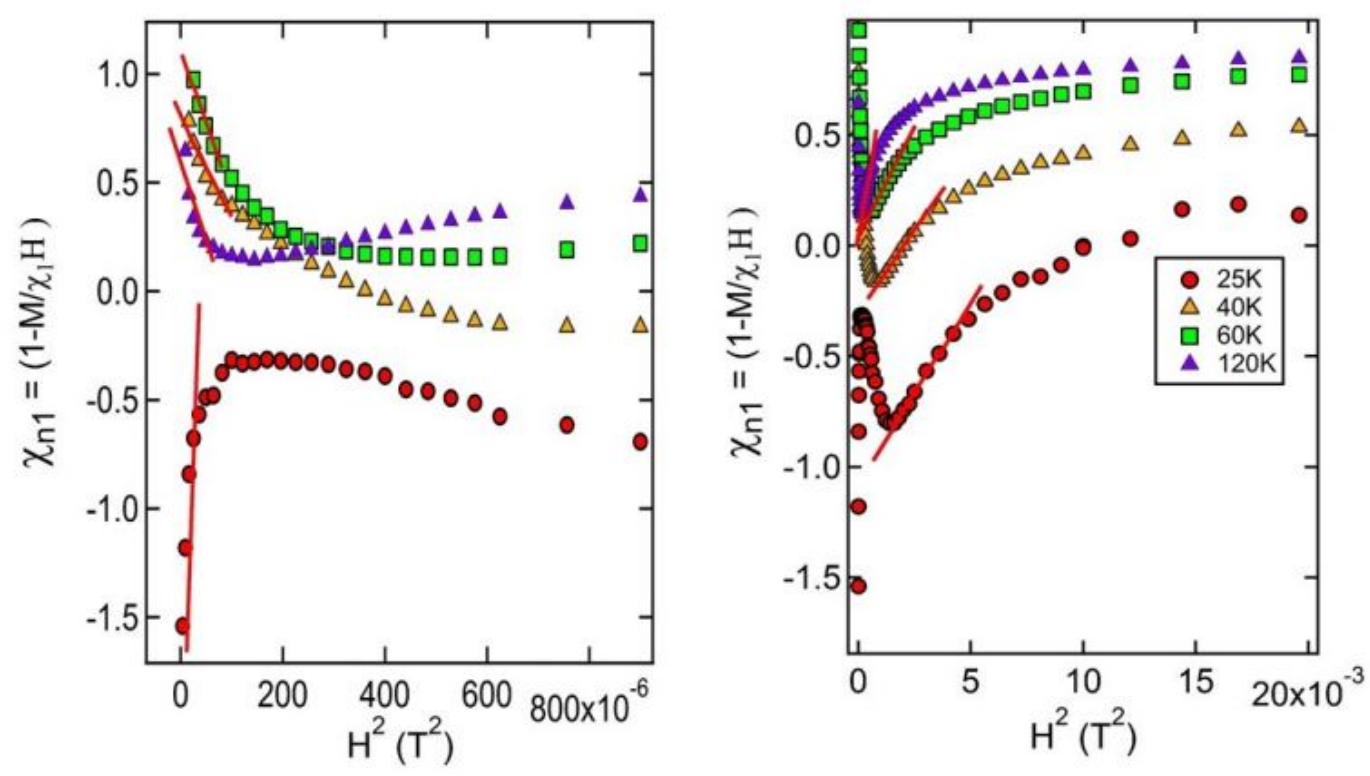

Figure 5.7: Plots of nonlinear susceptibilities, $\chi_{n 1}$, at few characteristic temperatures. $\chi_{n 1}$ is fitted in both low (left panel) and high field (right panel) regimes to obtain $\chi_{3}$. 

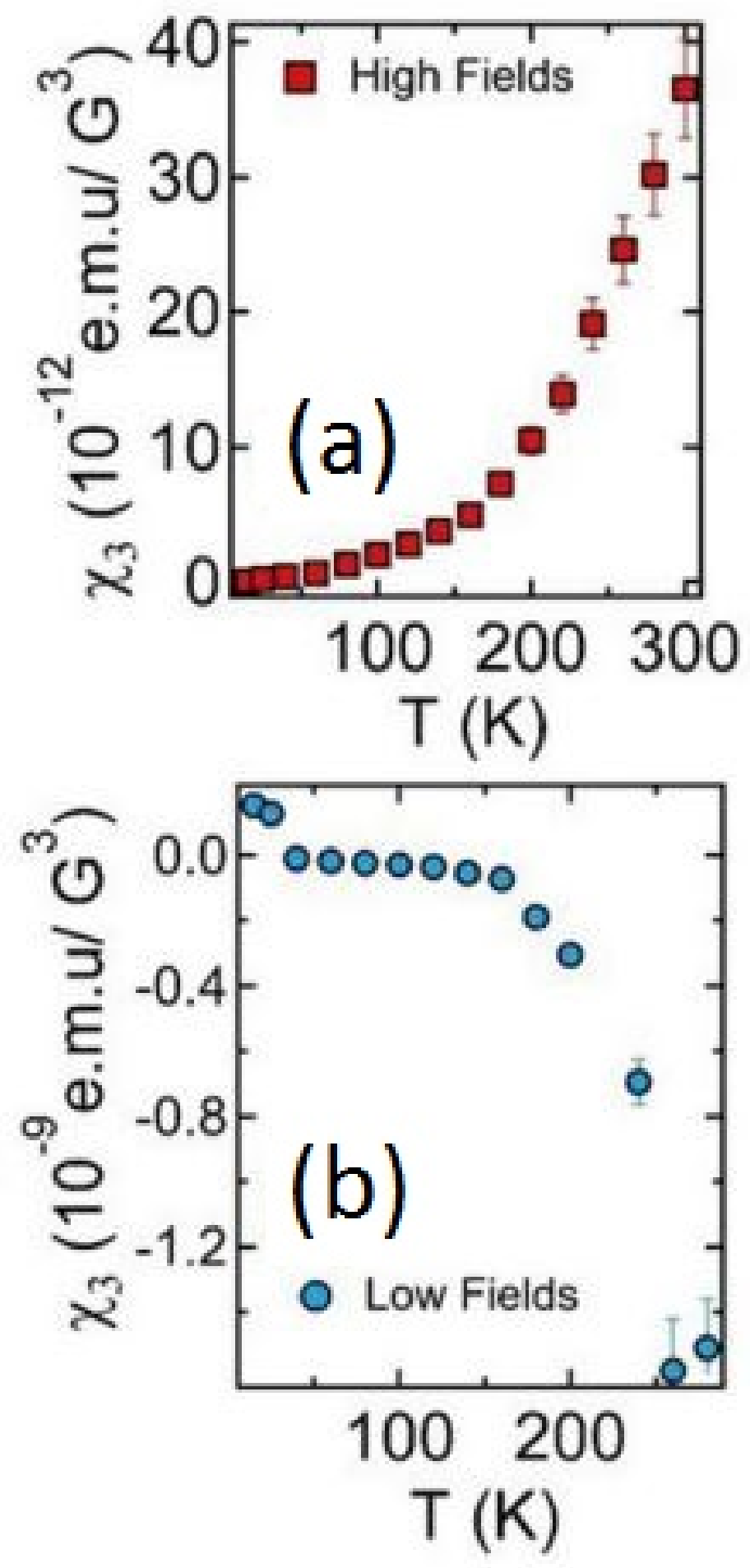

Figure 5.8: (a) and (b) Higher order susceptibility $\chi_{3}$ as a function of temperature across the crossover field. $\chi_{3}$ increases as a function of temperature in high field regime (a) and becomes more negative in low field regime 1 (b). 
To verify the equilibrium nature of magnetic phase transition to the spin solid state, first we extract the exponent $\gamma$ using the formalism, described above, in Eq. (5.7). For this purpose, the nonlinear susceptibility $\chi_{n 1}=a_{3} \chi_{1}^{2}$ is plotted as a function of $\tau$ for few different choices of spin solid transition temperatures $T_{s} \epsilon[25,35] \mathrm{K}$ in Fig. 5.9(a). e have fitted a fixed number of data points, in the divergence regime, on each curve using Eq. (5.7). Estimated $\gamma$ is found to vary in the range of $[1.7,2]$. The best fit is obtained for $T_{s}=29 \mathrm{~K}$, with the corresponding value of $|\gamma|=1.9$. The transition temperature $T_{s}$ is very close to the experimental value of $T=30 \mathrm{~K}$, as estimated from the previous dc susceptibility and electrical measurements. Also, the static critical exponent $\gamma$ is comparable to the value $(|\gamma| \simeq 2.25)$ found in system manifesting truly thermodynamic phase transition, such as interacting arrays of nano islands or spin freezing in canonical and geometrically frustrated systems. Similar analysis was performed in the low field regime (regime 1) below the characteristic crossover field. The best fit is obtained for the static critical exponent $|\gamma|=1.4$, see Fig.5.9(b). It seems that the crossover phenomenon, manifested by the change in the slope of $\gamma_{n 1}(T)$ as the system traverses across the transition temperature at a given field, does not affect the estimation of $\gamma$ and the transition temperature $T_{s}$ in the honeycomb lattice of ultra small elements.

Next, we determine another critical exponent $\delta$ by plotting $\left.\ln \left(\chi_{n 1}\right)\right)$ versus $\ln (H)$ at temperature near the spin solid transition. The experimental data is fitted using the asymptotic function in Eq.(5.8). As shown in Fig 5.9(c), a good fit to the data is obtained for the critical exponent $\delta=2.4$ in regime 2. Similar analysis in regime 1 at low field yields $|\delta|=2.5$, which is also similar in magnitude as found in the high field regime 2. Finally, we test the scaling behavior of nonlinear susceptibilities, as described by Eq. (5.3). If the magnetic phase transition to the spin solid state in artificial honeycomb lattice is indeed a true equilibrium phase transition, then the nonlinear susceptibilities should exhibit the scaling behavior due to the estimated 

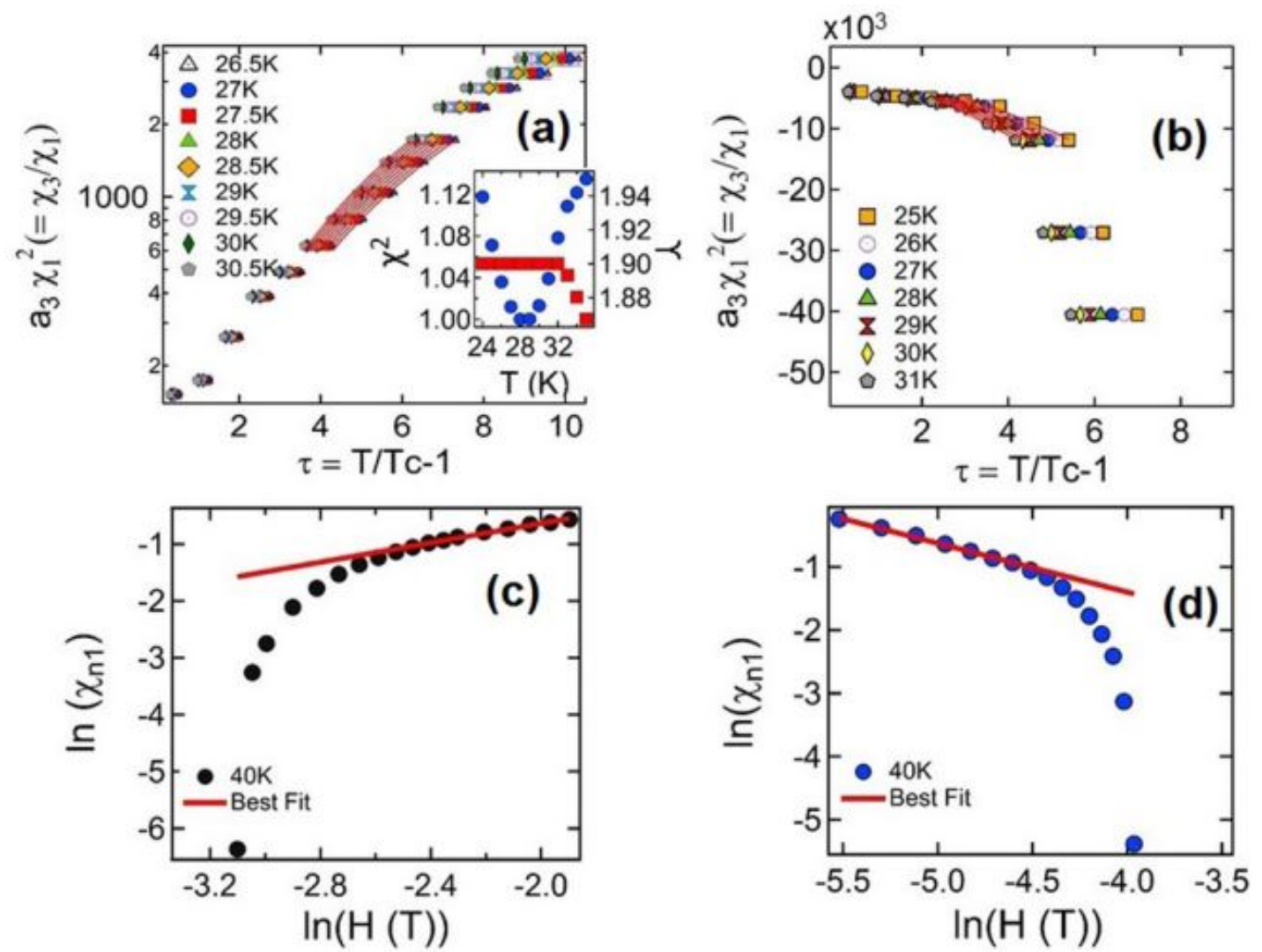

Figure 5.9: (a)To estimate the critical exponent, the coefficient a3 is plotted as a function of $\tau=\left(T / T_{s}\right)-1$ for different $T_{s}$ values, across the spin solid transition at $\mathrm{T}=30 \mathrm{~K} . \gamma$ is estimated by fitting the fixed number of points in the divergence regime of the curve using Eq. (5.7). Best fit to the experimental data is obtained for the critical exponent $|\gamma|=1.9$ (inset shows the plot of fitting parameter $\chi^{2}$ vs $\gamma$. (b) Similar analysis is performed for the low field regime 1, with estimated $|\gamma|=1.4$. In both regimes, best fit corresponds to spin solid transition at $T_{s}=29 \mathrm{~K}$. Nonlinear susceptibility $\chi_{n 1}$ is plotted as a function of field at temperature near Ts. Experimental data are fitted using the asymptotic function in Eq. (5.8) to obtain critical exponent $|\delta|(\mathrm{c})$ in high field regime $2, \sim 2.4$ and $(\mathrm{d})$ low field regime 1, 2.5. 
critical exponents. According to Eq. (5.9), for critical coefficients $|\gamma|=1.9$ and $\delta=2.4$, the magnetic order parameter critical exponent $\beta$ is $\simeq 1.4$. As shown in Fig. 5.7 the nonlinear susceptibilities at different temperatures do not exhibit the scaling collapse on one curve for the estimated exponents. To explore the scaling behavior further, we vary the critical exponents $\gamma, \delta$, and $\beta$ systematically. First we discuss the scaling in regime 2. A scaling behavior is observed for exponents $\delta=10$ and $|\gamma|=1.5$, see Fig.5.10 (a). Although exponent $|\gamma|$ is similar to the estimated value, scaling collapse of $\chi_{n 1}$ data only occurs for $\delta$ much larger than the estimated value. At large $\mathrm{x}$ values, some data scatter from the scaling curve due to the large errors associated with the smaller nonlinear susceptibilities.

We also tested the scaling behavior for intermediate values of $\delta \sim 4.75$, while keeping the coefficient $\gamma$ constant. The scaling of nonlinear susceptibilities improves as $\delta$ increases. However, the critical exponents do no follow the scaling relation, outlined in Eq. (5.9).

The scaling behavior was also tested for nonlinear susceptibilities in low field regime 1. For uniformity, we have used the estimated static critical exponents of $|\gamma|=1.4, \delta=2.5$, and $|\beta|=0.95$ for the scaling analysis. As shown in Fig. 5.11, the nonlinear susceptibilities do not scale for the calculated values of exponents. To our surprise, $\chi_{n 1}$ data at different temperature exhibit scaling characteristic for the similar set of exponents, $|\gamma|=1.4, \delta=10$, and $|\beta|=0.1$, that are used to obtain scaling collapse in the high field regime 2, see Fig. 5.10(b). Once again, the critical exponents do not satisfy the scaling relation in Eq. (5.9). It further confirms that the magnetic phase transition to the spin solid state is not thermodynamic in nature. The observed consistencies in the estimation of critical exponents as well as in the scaling analysis in two different regimes of $\chi_{n 1}$ constitute a unique aspect of the spin solid phase transition. It suggests that the nonlinear correction to magnetic susceptibility in spin solid phase is subtly similar to that in the high temperature phases. The dis- 
crepancies between the estimated values of the static critical exponents and that used for the scaling manifestation can be attributed, arguably, to the formation of small ferromagnetic clusters with short-range order at intermediate temperatures, which ultimately enhances $\chi_{n 1}$ considerably and led to strong but noncritical background temperature dependence. Similar behavior was previously observed in magnetic systems that exhibit non equilibrium phase transition [105].
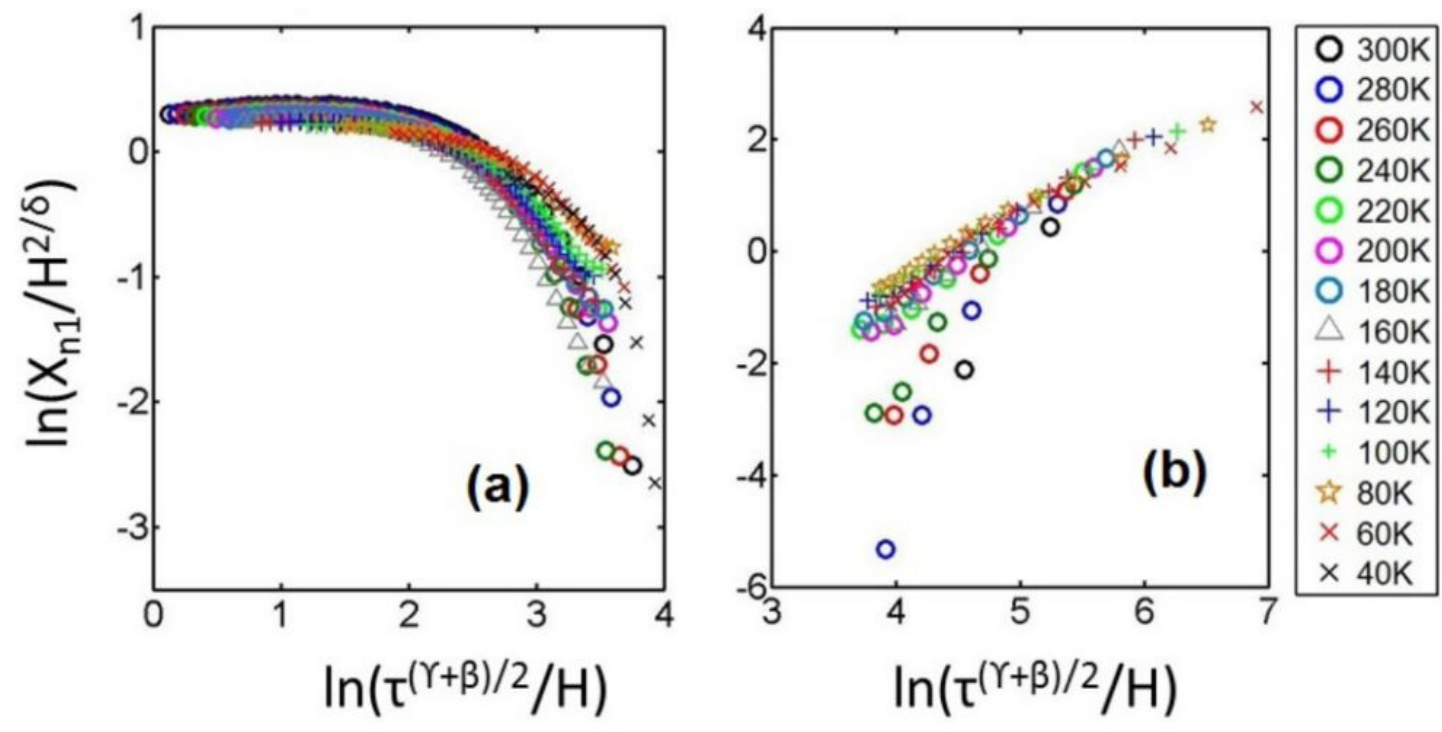

Figure 5.10: Scaling analysis of nonlinear susceptibilities in artificial honeycomb lattice. (a) Nonlinear susceptibilities exhibit scaling behavior for $|\gamma|=1.5, \delta=10$, and $|\beta|=0.1$. The critical scaling coefficients do not satisfy the scaling relation in Eq. (5.9). (b) Similar analysis was performed in the low field regime 1. Interestingly, the nonlinear susceptibilities exhibit scaling behavior for the same set of critical exponents, as in high field regime 2 . 


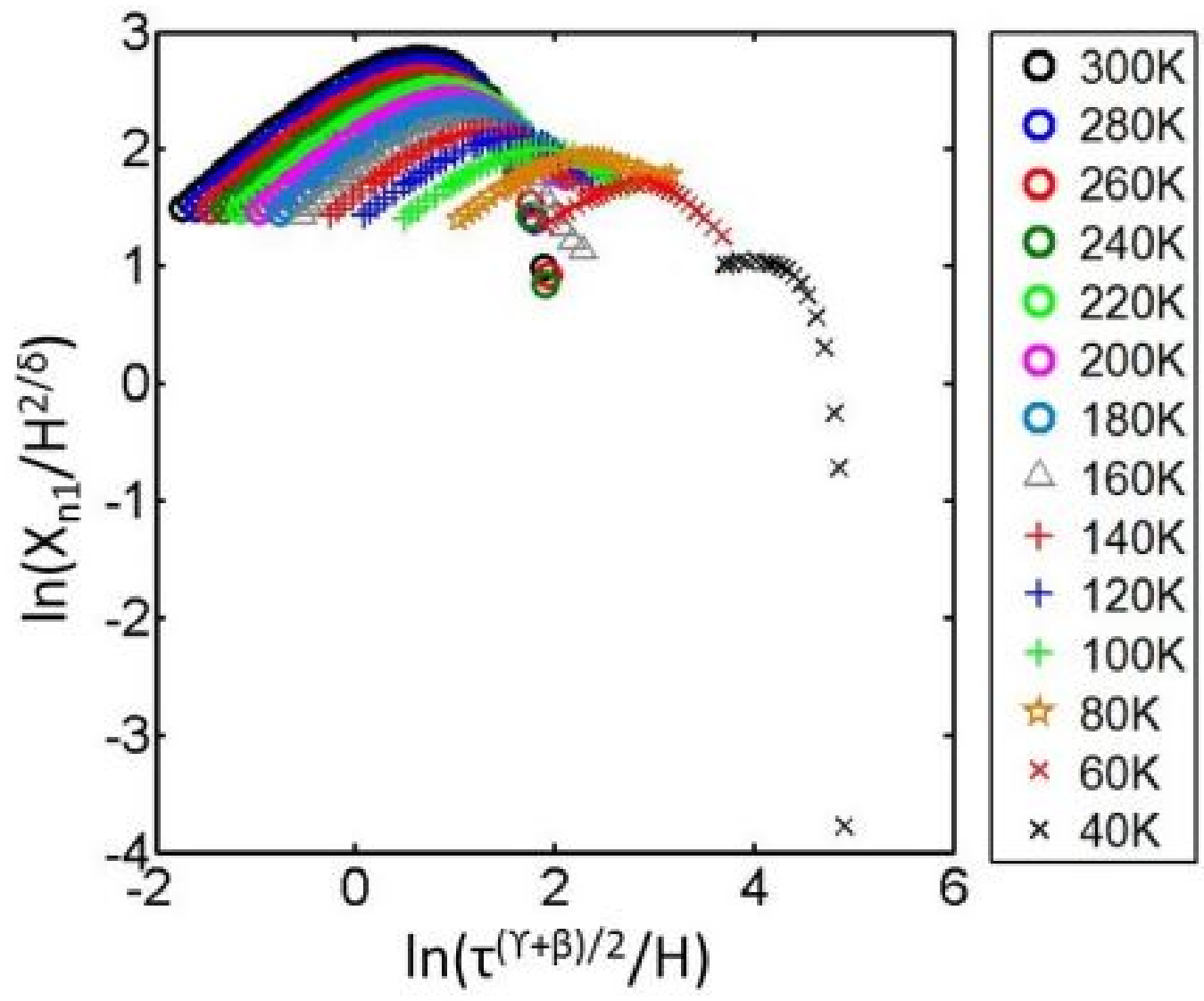

Figure 5.11: Scaling analysis of nonlinear susceptibilities, $\chi_{n 1}$, in artificial honeycomb lattice using the estimated exponents of $|\gamma|=1.9, \delta=2.4$ and $|\beta|=1.4$ in high field regime 2. Clearly, experimental data at different temperatures do not collapse on one curve. Thus, no scaling of $\chi_{n 1}$ is observed for the estimated exponents. 


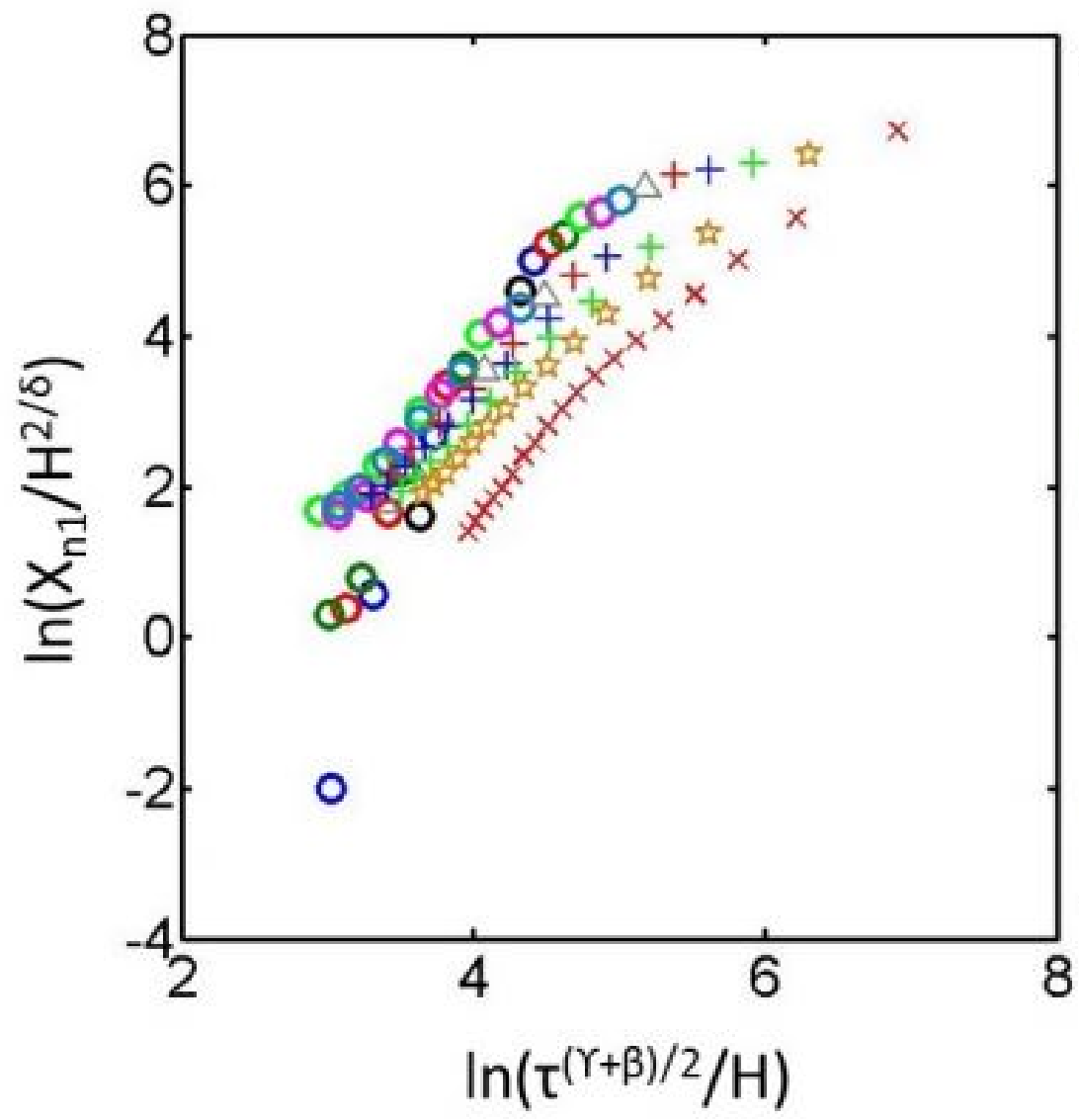

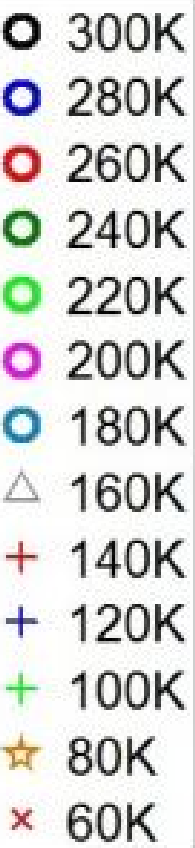

Figure 5.12: Scaling analysis of nonlinear susceptibilities, $\chi_{n 1}$, in low field regime 1 using the estimated exponents of $|\gamma|=1.4, \delta=2.5$ and $|\beta|=0.95$. Again, no scaling of $\chi_{n 1}$ at different temperatures is observed for the estimated exponents 


\subsection{Results and Discussion}

Our investigation of the equilibrium nature of magnetic phase transition in artificial honeycomb lattice has revealed two important properties that are not conventional in nature: first, the nonlinear susceptibility exhibits a crossover behavior in both temperature and magnetic field. The slope of $\chi_{n 1}$, which is used to determine the strength of the nonlinear correction to the overall magnetic susceptibility, is found to change from negative, at low field, to positive, at high field. Also, the net nonlinear susceptibility $\chi_{n 1}$ changes from positive to negative in temperature. This crossover occurs across the spin solid phase transition temperature at $T \simeq 30 K$. A magnetic phase transition is not known to depict such contrasting characteristic across the transition temperature. Clearly, the underlying magnetism in artificial honeycomb lattice does not fit congruently with the conventional understanding. Second, the experimental data do not exhibit scaling behavior for the estimated values of critical exponents. Rather, a scaling collapse of $\chi_{n 1}$ requires much larger value of the critical exponent $\delta$; not typically observed in a magnetic material with equilibrium phase transition. Also, the static critical exponents do not satisfy the conventional thermodynamic scaling relation. The overall scaling behavior suggests a non conventional nature of the transition, which can be arising either due to the finite spin dynamics in the system or, a distribution of relaxation times in short-range ordered magnetic clusters, such as spin ice order or the vortex loop type magnetic correlation across one honeycomb. A distribution of relaxation times in magnetic clusters is known to cause non conventional scaling behavior. The presence of spin dynamics or the distribution in spin relaxation rate, especially at low temperature, will result in finite entropy accumulation. It is worth pointing out that the large element size honeycomb lattice, with much larger dipolar interaction energy, may exhibit different nonlinear magnetic response. Further research works are highly desirable to fully understand the perplexing observations reported here as well as to explore the implication to 
large element size honeycomb lattice, especially in the disconnected structure. 


\section{Chapter 6}

\section{Summary and concluding remarks}

We have performed extensive measurements and analysis on various magnetic materials. We have found peculiar diamagnetism in NiSi which could be arising due to a minority superconducting phase present in the sample. We have performed tests to rule out the possibility of skin effect which might give rise to the unconventional frequency dependent diamagnetism. However, the tests show that the behavior is not due to skin effect. Overall, the kink in the electrical data, the diamagnetic response in magnetization measurement with irreversibility in ZFC and FC data as well as the decrease in magnetic signal with application of higher field lead to the conclusion that minor superconducting phase is present in the system. Growing a very thin film of NiSi would be an avenue to explore this property.

We have found a new way to study quantum magnetic properties in perovskite material by introducing artificial defects and creating frustration. In the case of $\mathrm{CaRuO}_{3}$, we have doped it with Co ions to create the dimers of artificial spin- $1 / 2$. This system tends to show singlet-to-triplet excitation at low temperature in absence of ordered spin correlation. Being able to create tailored frustrated systems gives us new ways to control the disorder and explore various other properties that might emerge. 
In $\mathrm{EuMn}_{2} \mathrm{As}_{2}$ we have found that the material undergoes through two magnetic transitions at $\sim 14 \mathrm{~K}$ and $\sim 135 \mathrm{~K}$. We have observed that the order parameter measurement shows the transition to be 3D Heisenberg type. We have modeled the elastic peaks of from the neutron scattering measurement and have associated the magnetic transition with ferromagnetic ordering of Mn along c-axis at $\sim 135 \mathrm{~K}$ and anti ferromagnetic transition of $\mathrm{Eu}$ ions along c-axis at $\sim 14 \mathrm{~K}$. Growing a larger single crystal sample would be very exciting to explore the dynamic properties of the material.

Finally, we have explored the nature of magnetic phase transition in 2D artificial honeycomb lattice. The system tends to go to the exotic spin solid state at low temperature. In a new observation,the spin solid transition coincides with insulating character in electrical measurements. We have studied the peculiar insulating property of $2 \mathrm{D}$ honeycomb lattice. We have found that this could be due to fractional electrical dimensionality. We have also studied the nature of the phase transition in artificial honeycomb lattice, where we have observed a non thermodynamic transition to the spin solid state, which is different than what was predicted. Further research is needed to understand this peculiar nature of phase transitions in this material. 


\section{Bibliography}

[1] V. Melfos, B. Helly, and P. Voudouris. The ancient greek names magnesia and magnetes and their origin from the magnetite occurrences at the mavrovouni mountain of thessaly, central greece. a mineralogicalgeochemical approach. Archaeological and Anthropological Sciences, 3:165-172, 2011.

[2] J.M.D.Coey. Rare-earth magnets. Endeavour, 19:146-151, 1995.

[3] J.M.D. Coey. Magnetism in future. Journal of Magnetism and Magnetic Materials, 226-230:2107 - 2112, 2001.

[4] James Clerk Maxwell. A Dynamical Theory of the Electromagnetic Field. Royal Society Publishing, London, first edition, 1865.

[5] S. Blundell. Magnetism in Condensed Matter. Oxford University Press, Great Clarendon Street, Oxford OX2 6DP, first edition, 2001.

[6] G. F. Hardy and J. K. Hulm. Superconducting silicides and germanides. Phys. Rev., 89:884, 1953.

[7] U. K. Robler, A. N. Bogdano, and C. Pfleiderer. Spontaneous skyrmion ground states in magnetic metals. Nature (London), 442:797, 2006.

[8] S. Krannich, Y. Sidis, D. Lamago, R. Heid, J.-M. Mignot, H. V. L ohneysen, A. Ivanov, P. Steffens, T. Keller, E. Goering L. Wang, and F. Weber. Mag- 
netic moments induce strong phonon renormalization in FeSi. Nat. Commun., $6: 8961,2015$.

[9] N. Manyala, Y. Sidis, J. DiTusa, G. Aeppli, D. Young, and Z. Fisk. Large anomalous hall effect in a silicon-based magnetic semiconductor. Nat. Mater., $3: 255,2004$.

[10] O. Delaire, K. Marty, M. Stone, P. Kent, M. Lucas, D. Abernathy, D. Mandrus, and B. Sales. Phonon softening and metallization of a narrow-gap semiconductor by thermal disorder. Proc. Natl. Acad. Sci. USA, 108:4725, 2011.

[11] H. Iwai, T. Ohguro, and S. Ohmi. NiSi salicide technology for scaled CMOS. Microelectron. Eng., 60:157, 2002.

[12] T. Morimoto, T. Ohguro, S. Momose, T. Iinuma, I. Kunishima, K. Suguro, I. Katakabe, H. Nakajima, M. Tsuchiaki, and M. Ono et al. Self-aligned nickelmono-silicide technology for highspeed deep submicrometer logic CMOSULSI. Electron. Dev., 42:915, 1995.

[13] B. Meyer, U. Gottlieb, O. Laborde, H. Yang, J. Lasjaunia, A. Sulpice, and R. Madar. Intrinsic properties of NiSi. J. Alloys Compd., 262-263:235, 1997.

[14] A. Franciosi, J. H. Weaver, and F. A. Schmidt. Electronic structure of nickel silicides $\mathrm{Ni}_{2} \mathrm{Si}, \mathrm{NiSi}$, and NiSi. Phys. Rev. B, 26:546, 1982.

[15] David Pines and David Bohm. A collective description of electron interactions: Ii. collective vs individual particle aspects of the interactions. Phys. Rev., 85:338-353, 1952.

[16] N. Kikugawa, L. Balicas, and A. P. Mackenzie. Physical properties of singlecrystalline $\mathrm{CaRuO}_{3}$ grown by a floating-zone method. J. Phys. Soc. Jpn., 78:014701, 2009. 
[17] L. Klein, L. Antognazza, T. H. Geballe, M. R. Beasley, and A. Kapitulnik. Possible non-fermi-liquid behavior of $\mathrm{CaRuO}_{3}$. Phys. Rev. B, 60:3, 1999.

[18] H. Mukunda, K. Ishida, Y.Kitaoka, K. Asayama, R. Kanno, and M. Takano. Spin fluctuations in the ruthenium oxides $\mathrm{ruo}_{2}, \mathrm{srruo}_{3}, \mathrm{caruo}_{3}$, and $\mathrm{sr}_{2} \mathrm{ruo}_{4}$ probed by ru nmr. Phys. Rev. B, 60:17, 1999.

[19] J. Gunasekera, L. Harriger, A. Dahal, T. Heitmann, G. Vignale, and D. K. Singh. Magnetic fluctuations driven insulator-to-metal transition in Ca $\left(\operatorname{Ir}_{1 x} R u_{x}\right) O_{3}$. Sci. Rep., 5:18047, 2015.

[20] P. A. Lee. From high temperature superconductivity to quantum spin liquid: progress in strong correlation physics. Rep. Prog. Phys., 71:012501, 2008.

[21] S. Sachdev. Quantum criticality: Competing ground states in low dimensions. Science, 288:475, 2000.

[22] H. Yao and S. A. Kivelson. Exact spin liquid ground states of the quantum dimer model on the square and honeycomb lattices. Phys. Rev. Lett., 108:247206, 2012.

[23] P.W. Anderson. Resonating valence bonds: A new kind of insulator?

[24] L. Balents. Spin liquids in frustrated magnets. Nature, 464:199, 2010.

[25] T. Mizusaki and M. Imada. Gapless quantum spin liquid, stripe, and antiferromagnetic phases in frustrated hubbard models in two dimensions. Phys. Rev. B, 74:014421, 2006.

[26] Z. Y. Meng, T. C. Lang, S. Wessel, F. F. Assaad, and A. Muramatsu. Quantum spin liquid emerging in two-dimensional correlated dirac fermions. Nature, 464:847, 2010.

[27] S. Yan, D. A. Huse, and S. R. White. Spin-liquid ground state of the $s=1 / 2$ kagome heisenberg antiferromagnet. Science, 332:1173-1176, 2011. 
[28] T. Han, J. Helton, D. Nocera S. Chu, J. Rodriguez, C. Broholm, and Y. S. Lee. Fractionalized excitations in the spin-liquid state of a kagome-lattice antiferromagnet. Nature, 492:406, 2012.

[29] S. H. Lee, H. Kikuchi, Y. Qiu, B. Lake, Q. Huang, K. Habicht, and K. Kiefer. Quantum-spin-liquid states in the two-dimensional kagome antiferromagnets $\mathrm{Zn}_{x} \mathrm{Cu}_{4 x}(\mathrm{OD})_{6} \mathrm{Cl}_{2}$. Nat. Mater., 6:853, 2007.

[30] G. Xu, C. Broholm, D. H. Reich, and M. A. Adams. Triplet waves in a quantum spin liquid. Phys. Rev. Lett., 84:4465, 2000.

[31] M. Mourigal, W. T. Fuhrman, J. P. Sheckelton, and D. L. Abernathy T. M. McQueen C. L. Broholm A. Wartelle, J. A. Rodriguez-Rivera. Molecular quantum magnetism in $\mathrm{LiZn}_{2} \mathrm{Mo}_{3} \mathrm{O}_{8}$. Phys. Rev. Lett., 112:027202, 2014.

[32] D. C. Johnston. The puzzle of high temperature superconductivity in layered iron pnictides and chalcogenides. Adv. Phys, 59:803, 2010.

[33] G. R. Stewart. Superconductivity in iron compounds. Rev. Mod. Phys., 83:1589, 2011.

[34] D. J. Scalapino. A common thread: The pairing interaction for unconventional superconductors. Rev. Mod. Phys., 84:1383, 2012.

[35] E. Dagotto. Colloquium: The unexpected properties of alkali metal iron selenide superconductors. Rev. Mod. Phys., 85:849, 2013.

[36] R. M. Fernandes, A. V. Chubukov, and J. Schmalian. What drives nematic order in iron-based superconductors? Nat. Phys, 10:97, 2014.

[37] H. Hosono and K. Kuroki. Iron-based superconductors: Current status of materials and pairing mechanism. Physica C, 514:399, 2015. 
[38] J. Tranquada, H. Woo, T. G. Perring, H. Goka, G. D. Gu, G. Xu, M. Fujita, and K. Yamada. Quantum magnetic excitations from stripes in copper oxide superconductors. Nature (London), 429:534, 2004.

[39] P. Gegenwart, Q. Si, and F. Steglich. Quantum criticality in heavy-fermion metals. Nat. Phys., 4:186, 2008.

[40] Z. Fisk, D. W. Hess, C. J. Pethick, D. Pines, J. L. Smith, J. D. Thompson, and J. O. Willis. Heavy-electron metals: New highly correlated states of matter. Science, 239:33, 1988.

[41] P. Coleman and A. Schofield. Quantum criticality. Nature, 433:226, 2005.

[42] S. Sachdev. Quantum Criticality. Cambridge University Press, New York, 1999.

[43] C. M. Varma. Mixed-valence compounds. Rev. Mod. Phys., 48:219, 1976.

[44] V. K. Anand, D. T. Adroja, A. Bhattacharyya, U. B. Paramanik, P. Manuel, A. D. Hillier, D. Khalyavin, and Z. Hossain. sr and neutron diffraction investigations on the reentrant ferromagnetic superconductor $E u\left(F e_{0.86} I r_{0.14}\right)_{2} A s_{2}$. Phys. Rev. B, 91:094427, 2015.

[45] P. Schlottmann. Dynamical spin-susceptibility of itinerant heavy electrons with nested fermi surface. Phys. Rev. B, 75:205108, 2007.

[46] D. K. Singh, A. Thamizhavel, S. Chang, J. W. Lynn, D. A. Joshi, S. K. Dhar, and S. Chi. Coupling of field-induced spin fluctuations and spin-density wave in intermetallic $\mathrm{CeAg}_{2} \mathrm{Ge}_{2}$. Phys. Rev. B, 84:052401, 2011.

[47] P. Das, N. Sangeetha, A. Pandey, Z. Benson, T. Heitmann, D. Johnston, A. Goldman, and A. Kreyssig. Collinear antiferromagnetism in trigonal srmn2as2 revealed by single-crystal neutron diffraction. J. Phys.: Condens. Matter, 29:035802, 2017. 
[48] U. B. Paramanik, R. Prasad, C. Geibel, and Z. Hossain. Itinerant and localmoment magnetism in $\mathrm{EuCr}_{2} \mathrm{As}_{2}$ single crystals. Phys. Rev. B, 89:144423, 2014 .

[49] S. Graser, T. A. Maier, P. J. Hirschfeld, and D. J. Scalapino. Near-degeneracy of several pairing channels in multiorbital models for the Fe pnictides. New J. Phys., 11:025016, 2009.

[50] R. Ruhl and W. Jeitschko. New pnictides with $\mathrm{Ce}_{2} \mathrm{O}_{2} \mathrm{~S}$-type structure. Mater. Res. Bull., 14:513, 1979.

[51] V. K. Anand and D. C. Johnston. Metallic behavior induced by potassium doping of the trigonal antiferromagnetic insulator $E u M n_{2} A s_{2}$. Phys. Rev. B, 94:014431, 2016.

[52] S. Stankovich, D. Dikin, G. Dommett, K. Kohlhaas, E. Zimney, E. Stach, R. Piner, S. Nguyen, and R. Ruoff. Graphene-based composite materials. Nature, 442:282, 2006.

[53] B. Feng, Z. Ding, S. Meng, Y. Yao, X. He, P. Cheng, L. Chen, and K. Wu. Evidence of silicene in honeycomb structures of silicon on $A g(111)$. Nano Lett., 12:3507, 2012.

[54] C. Ataca and S. Ciraki. Functionalization of single-layer $M o S_{2}$ honeycomb structures. J. Phys. Chem. C, 115:13303, 2011.

[55] C. Nisoli, R. Moessner, and P. Schiffer. Colloquium: Artificial spin ice: Designing and imaging magnetic frustration. Rev. Mod. Phys., 85:1473, 2013.

[56] M. Tanaka, E. Saitoh, H. Miyajima, T. Yamaoka, and Y. Iye. Magnetic interactions in a ferromagnetic honeycomb nanoscale network. Phys. Rev. B, 73:052411, 2006. 
[57] W. R. Branford, S. Ladak, D. E. Read, K. Zeissler, and L. F. Cohen. Emerging chirality in artificial spin ice. Science, 335:1597, 2012.

[58] G. Chern and O. Tchernyshyov. Magnetic charge and ordering in kagome spin ice. Phil. Trans. R. Soc. A, 370:5718, 2012.

[59] G. Moller and R. Moessner. Emerging chirality in artificial spin ice. Phys. Rev. $B, 80: 140409(\mathrm{R}), 2009$.

[60] G. W. Chern, P. Mellado, and O. Tchernyshyov. Two-stage ordering of spins in dipolar spin ice on the kagome lattice. Phys. Rev. Lett., 106:207202, 2011.

[61] Y. Kamihara, H. Hiramatsu, M. Hirano, R. Kawamura, H. Yanagi, T. Kamiya, , and H. Hosono. Iron-based layered superconductor:LaOFeP. J. Am. Chem. Soc., 128:10012, 2006.

[62] F.C. Hsu, J. Luo, K. Yeh, T. Chen, T. Huang, P. Wu, Y. Lee, Y. Huang, Y. Chu, D. Yan, and M. Wu. Superconductivity in the $P b O$-type structure a-FeSe. Proc. Natl. Acad. Sci. USA, 105:14262, 2008.

[63] J. M. Tomczak, K. Haule, and G. Kotliar. Signatures of electronic correlations in iron silicide. Proc. Natl. Acad. Sci. USA, 109:3243, 2012.

[64] A. Krzton-Maziopa, V. Svitlyk, E. Pomjakushina, R. Puzniak, and K. Conder. Superconductivity in alkali metal intercalated iron selenides.

[65] L. Chen. Metal silicides: An integral part of microelectronics. JOM, 57:24, 2005.

[66] F. Gomory. Characterization of high-temperature superconductors by $A C$ susceptibility measurements. Supercond. Sci. Technol., 10:523, 1997.

[67] QDPPMS. Heat capacity option users manual. Part Number 1085-150, H-1. 
[68] R. Singh, R. Lal, U. C. Upreti, D. K. Suri, A. V. Narlikar, V. P. S. Awana, J. Albino Aguiar, and M. Shahabuddin. Superconductivity in zn-doped tetragonal $\mathrm{LaBaCaCu} \mathrm{O}_{7}$ systems. Phys. Rev. B, 55:1216, 1997.

[69] M. D. Vannette. Dynamic magnetic susceptibility of systems with long-range magnetic order. Ph.d. thesis, Iowa State University, 2009.

[70] V. B. Geshkenbein, V. M. Vinokur, and R. Fehrenbacher. ac absorption in the high-Tc superconductors: Reinterpretation of the irreversibility line. Phys. Rev. B, 43:3748(R), 1991.

[71] T. Chatterjil. Neutron Scattering from Magnetic Materials. Elsevier Science, first edition, 2006.

[72] G. Xu, Z. Xu, and J. M. Tranquada. Absolute cross-section normalization of magnetic neutron scattering data. Rev. Sci. Instrum., 84:083906, 2013.

[73] B. Lake, G. Aeppli, T. Mason, A. Schr oder, D. McMorrow, K. Lefmann, M. Isshiki, M. Nohara, H. Takagi, and S. Hayden. Spin gap and magnetic coherence in a clean high-temperature superconductor. Nature (London), 400:43, 1999.

[74] C. Kittel. Introduction to Solid State Physics. Fractals in Biology and Medicine. John Wiley and Sons, USA, 2005, 8 edition, 2005.

[75] P. Ehrenfest. Superconductivity of compacted platinum powder at very low temperatures. Physica (The Hague), 5:388, 1925.

[76] W. J. De Haas. Diamagnetism, field strength, and crystal structure. Nature, 127:335, 1931.

[77] Y. Hasegawa, P. Lederer, T. M. Rice, and P. B. Wiegmann. Theory of electronic diamagnetism in two-dimensional lattices. Phys.Rev.Lett., 63:907, 1989. 
[78] T. Hirose, Y. Okamoto, J.-I Yamaura, and Z. Hiroi. Large diamagnetic susceptibility from petit fermi surfaces in $L_{a} V_{2} A l_{20}$. J. Phys. Soc. Jpn., 84:113701, 2015.

[79] D. Podolsky, A. Paramekanti, Y. Kim, and T. Senthil. Mott transition between a spin-liquid insulator and a metal in three dimensions. Phys. Rev. Lett., 102:186401, 2009.

[80] S. Nakatsuji, S. Nakatsuji, K. Kuga, K. Kimura, R. Satake, N. Katayama, E. Nishibori, H. Sawa, R. Ishii, M. Hagiwara, F. Bridge, T. U. Ito, W. Higemoto, Y. Karaki, M. Halim, A. A. Nugroho, J. A. Rodriguez-Rivera, M. A. Green, and C. Broholm. Spin-orbital short-range order on a honeycomb-based lattice. Science, 336:559, 2012.

[81] R. Coldea, D. A. Tennant, A. M. Tsvelik, and Z. Tylczynski. Experimental realization of a $2 D$ fractional quantum spin liquid. Phys. Rev. Lett., 86:1335, 2001.

[82] H. Mukuda, K. Ishida, Y. Kitaoka, K. Asayama, R. Kanno, and M. Takano. Spin fluctuations in the ruthenium oxides $\mathrm{RuO} \mathrm{O}_{2}, \mathrm{SrRuO}_{3}, \mathrm{CaRuO}_{3}, \mathrm{andSr}_{2} \mathrm{RuO}_{4}$ probed by Ru NMR. Phys. Rev. B, 60:12279, 1999.

[83] L. F. Feiner, A. M. Oles, and J. Zaanen. Quantum melting of magnetic order due to orbital fluctuations. Phys. Rev. Lett., 78:2799, 1997.

[84] M. B. Stone, I. A. Zaliznyak, T. Hong, C. L. Broholm, and D. H. Reich. Quasiparticle breakdown in a quantum spin liquid. Nature, 440:187, 2006.

[85] M.J. Lawler, A. Paramekanti, Y.B. Kim, and L. Balents. Gapless spin liquids on the three-dimensional hyperkagome lattice of $n_{4} \mathrm{ir}_{3} \mathrm{O}_{8}$. Phys. Rev. Lett., 101:197202, 2008. 
[86] V. K. Anand, P. K. Perera, A. Pandey, R. J. Goetsch, A. Kreyssig, and D. C. Johnston. Crystal growth and physical properties of $\mathrm{SrCu}_{2} \mathrm{As}_{2}, \mathrm{SrCu}_{2} \mathrm{Sb}_{2}, \mathrm{andBaCu}_{2} \mathrm{Sb}_{2}$. Phys. Rev. B, 85:214523, 2012.

[87] Information about neutron absorption coefficient can be found at https://www.ncnr.nist.gov/instruments/bt1/neutron.html.

[88] A. Pelissetto and E. Vicari. Critical phenomena and renormalization-group theory. Phys. Rep., 368:549, 2002.

[89] G. Shirane, J. Tranquada, and S. Shapiro. Neutron Scattering with a Triple Axis Spectrometer: Basic Techniques. Cambridge University Press, New York, 2002.

[90] H. v. Lohneysen, A. Risch, M. Vojta, and P. Wolfle. Fermi-liquid instabilities at magnetic quantum phase transitions. Rev. Mod. Phys., 79:1015, 2007.

[91] D. K. Singh, A. Thamizhavel, J. W. Lynn, and S. K. Dharand T. Hermann. Multiple magnetic structures of correlated ce ions in intermetallic $\mathrm{CeAu}_{2} \mathrm{Ge}_{2}$. Phys. Rev. B, 86:060405(R), 2012.

[92] B. Summers, Y. Chen, A. Dahal, and D. K. Singh. New description of evolution of magnetic phases in artificial honeycomb lattice. Sci. Rep., 7:16080, 2017.

[93] V. Kapaklis, U. Arnalds, A. Farhan, R. Chopdekar, A. Balan, A. Scholl, L. J. Heyderman, and B. Hjorvarsson. Thermal fluctuations in artificial spin ice. Sci. Rep., 7:16080, 2017.

[94] Y. Qi, T. Brintlinger, and J. Cumings. Direct observation of the ice rule in an artificial kagome spin ice. Phys. Rev. B, 77:094418, 2008. 
[95] A. Glavic, B. Summers, A. Dahal, J. Kline, W. Van Herck, A. Sukhov, A. Ernst, and D. K. Singh. Spin solid versus magnetic charge ordered state in artificial honeycomb lattice of connected elements. Adv. Sci., 5:1700856, 2018.

[96] L. G. Aslamozov and A. I. Larkins. The influence of fluctuation pairing of electrons on the conductivity of normal metal. Phys. Lett. A, 26:238, 1968.

[97] R. Ivens, R. Wernhardt, M. Rosenberg, S. Losch, and S. Sack. Superconducting

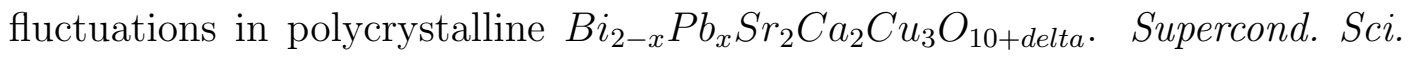
Technol., 5:16, 1992.

[98] P. Clippe, C. Laurent, S. K. Patapis, and M. Ausloos. Superconductivity fluctuations in electrical and thermoelectrical properties of granular ceramic superconductors: Homogeneous versus fractal behavior. Phys. Rev. B, 42:8611, 1990.

[99] A. L. Efros and B. I. Shklovskii. Coulomb gap and low temperature conductivity of disordered systems. J. Phys. C: Solid State Phys., 8:L49, 1975.

[100] P. Mellado, O. Petrova, Y. Shen, and O. Tchernyshyov. Dynamics of magnetic charges in artificial spin ice. Phys. Rev. Lett., 105:187206, 2010.

[101] Z. Budrikis, P. Politi, and R. L. Stamps. Diversity enabling equilibration: Disorder and the ground state in artificial spin ice. Phys.Rev.Lett., 107:217204, 2011.

[102] T. Jonsson, P. Svedlindh, and M. F. Hansen. Static scaling on an interacting magnetic nanoparticle system. Phys.Rev.Lett., 81:3976, 1998.

[103] D. C. Johnston. Magnetic susceptibility scaling in $\mathrm{La}_{2 x} S r_{x} C u O_{4 y}$. Phys.Rev.Lett., 62:957, 1989. 
[104] B. Martinez, A. Labarta, R. Rodrguez-Sol, and X. Obradors. Magnetic transition in highly frustrated $\mathrm{SrCr}_{8} \mathrm{Ga}_{4} \mathrm{O}_{19}$ : The archetypal kagome system. Phys. Rev. B., 50:15779, 1994.

[105] K. Binder. On the behavior of static susceptibilities in spin glasses. Z. Phys. B, 48:319, 1982 . 


\section{PUBLICATIONS}

Y. Chen, B. Summers, A. Dahal (Equal Contributor), V. Lauter, G. Vignale, D. K. Singh, Field and current control of the electrical conductivity of an artificial two-dimensional honeycomb lattice, Adv. Mater. 2019, 1808298 (2019).

Ashutosh Dahal, Y. Chen, T. Heitmann, S. K. Dhar, and D. K. Singh, Spin correlation in trigonal $\mathrm{EuMn}_{2} \mathrm{As}_{2}$, Physical Review B 99, 085135 (2019).

Y. Chen, A. Dahal, J. Rodriguez, G. Xu, L. Harriger, T. Heitmann, V. Dugaev, A. Ernst, D. J. Singh and D. K. Singh, Quantum continuum fluctuation in glassy perovskite $\mathrm{Ca}\left(\mathrm{Co}_{0.15} \mathrm{Ru}_{0.85}\right) 0_{3}$, Under review 2018; Preprint available on cond-mat arXiv- arXiv:1807.05404

Ashutosh Dahal, Y. Chen, B. Summers, and D. K. Singh, Scaling of nonlinear susceptibilities in artificial permalloy honeycomb lattice, Phys. Rev. B 97, 214420 (2018).

B. Summers, A. Dahal, and D. K. Singh, Magnetic Diode Behavior at Room Temperature in 2D Honeycombs, Adv. Electron. Mater. 4, 1700500 (2018).

Ashutosh Dahal, B. Summers, and D. K. Singh, Fractional electrical dimensionality in the spin solid phase of artificial honeycomb lattice, Appl. Phys. Lett. 112, 062407 (2018).

B. Summers, L. Debeer-Schmitt, A. Dahal, P. Kamschroeder , J. Gunasekera, and D. K. Singh, Temperature-dependent magnetism in artificial honeycomb lattice of connected elements, Phys. Rev. B 97, 014401 (2018).

A. Glavic, B. Summers, A. Dahal, J. Kline, W. Van Herck, A. Sukhov, A. Ernst, and D. K. Singh, Spin Solid versus Magnetic Charge Ordered State in Artificial Honeycomb Lattice of Connected Elements, Adv. Sci. 5, 1700856 (2018).

J. Gunasekera, A. Dahal, Y. Chen, T. Heitmann, J. Rodriguez-Rivera, L. Harriger, S. Thomas, V. Dugaev, A. Ernst, and D. K. Singh, Quantum magnetic properties in perovskite with Anderson localized artificial spin-1/2, Adv. Sci. 5, 1700978 
(2018).

B. Summers, Y. Chen, A. Dahal, and D. K. Singh, New Description of Evolution of Magnetic Phases in Artificial Honeycomb Lattice, Sci. Rep. 7, 16080 (2017).

Ashutosh Dahal, J. Gunasekera, and D. K. Singh, Possible superconductivity in chemically doped $\mathrm{CrSb}_{1+\delta}$, Phys. Status Solidi RRL 11: 1700211 (2017).

Ashutosh Dahal, J. Gunasekera, L. Harriger, D. J. Singh, and D. K. Singh, Intermediate regime between metal and superconductor below $\mathrm{T}=100 \mathrm{~K}$ in $\mathrm{NiSi}$, Phys. Rev. B 94, 184516 (2016).

J. Gunasekera, L. Harriger, A. Dahal, A. Maurya, T. Heitmann, S. M. Disseler, A. Thamizhavel, S. Dhar, D. J. Singh, and D. K. Singh, Electronic nature of the lock-in magnetic transition in $\mathrm{CeXAl}_{4} \mathrm{Si}_{2}$, Phys. Rev. B 93, 155151(2016).

Ashutosh Dahal, J. Gunasekera; L. Harringer, D. K. Singh, and D. J. Singh, Properties of Metallic NiSi and Other Nickel Silicides, J. Alloys Compd., 672, 110 -116 (2016).

J. Gunasekera, L. Harriger,A. Dahal, T. Heitmann, G. Vignale, and D. K. Singh, Magnetic fluctuations driven insulator-to-metal transition in $\mathrm{Ca}\left(\mathrm{Ir}_{1-x} \mathrm{Ru}_{x}\right) \mathrm{O}_{3}$, Sci. Rep. 5, 18047 (2015).

J. Gunasekera, L. Harriger, T. Heitmann,A. Dahal , H. Knoll, and D. K. Singh, Quasilocal critical nature of cooperative paramagnetic fluctuations in $\mathrm{CaRuO}_{3}$ metal, Phys. Rev. B 91, 241103(R) (2015). 


\section{VITA}

Ashutosh Dahal was born in Katari-8 of Udayapur district in Nepal. He pursued physics and mathematics as an undergraduate at William Jewell College, Liberty, MO, USA and graduated in 2013. He joined University of Missouri, Department of Physics and Astronomy, in August 2013. He started working with professor Deepak K. Singh from Summer of 2014. He will receive his PhD degree in July 2019. He will start working for Intel corporation from September 2019 as Thin Film Module Engineer. 